Aus der Klinik für Augenheilkunde

(Prof. Dr. med. H. Hoerauf)

der Medizinischen Fakultät der Universität Göttingen

\title{
Die operative Versorgung der kindlichen Katarakt. Eine retrospektive Datenanalyse über 25 Jahre
}

\author{
INAUGURAL-DISSERTATION \\ zur Erlangung des Doktorgrades \\ der Medizinischen Fakultät der \\ Georg-August-Universität zu Göttingen
}

vorgelegt von

Jan Moritz Messer

aus

Hamburg

Göttingen 2018 
Dekan:

Referent/in

Ko-Referent/in:
Prof. Dr. rer. nat. H.K. Kroemer

Prof. Dr. med. N. Feltgen

Priv.-Doz. Dr. med. Hendrik Rosewich

Datum der mündlichen Prüfung: 19. März 2019 
Hiermit erkläre ich, die Dissertation mit dem Titel "Die operative Versorgung der kindlichen Katarakt. Eine retrospektive Datenanalyse über 25 Jahre" eigenständig angefertigt und keine anderen als die von mir angegebenen Quellen und Hilfsmittel verwendet zu haben.

Göttingen, den 


\section{Inhaltsverzeichnis}

Abbildungsverzeichnis ............................................................................................. III

Tabellenverzeichnis ....................................................................................... III

Abkürzungsverzeichnis .....................................................................................

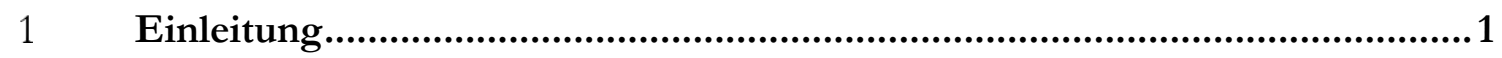

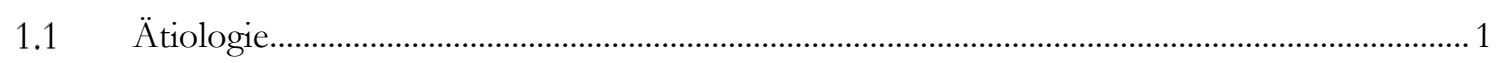

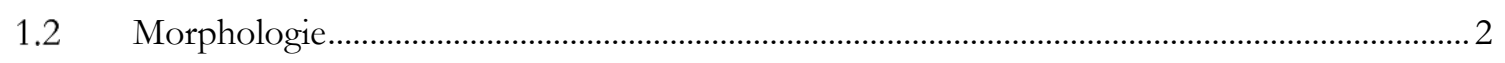

1.2.1 Embryonale Linsenentwicklung..................................................................................

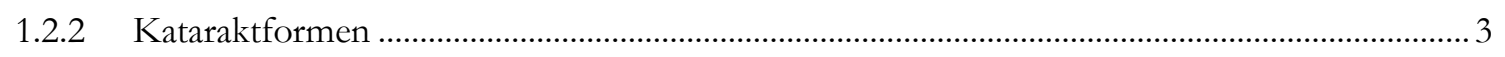

1.3 Amblyopie und kongenitale Katarakt ………................................................................... 5

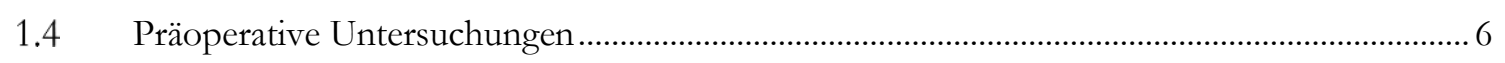

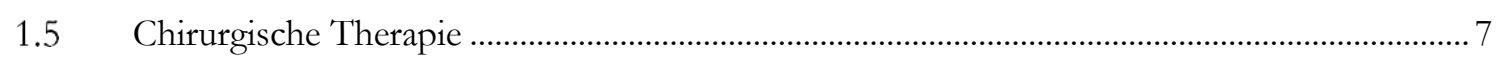

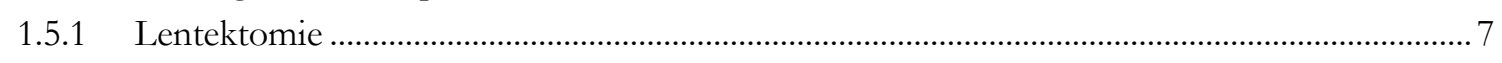

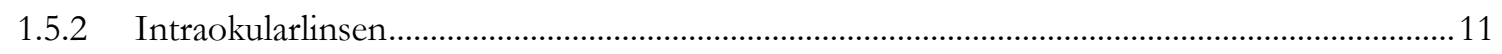

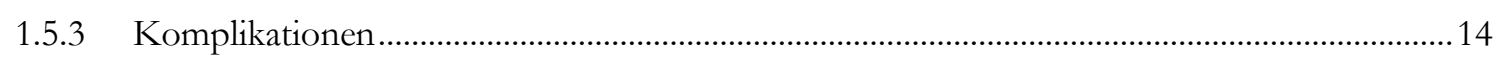

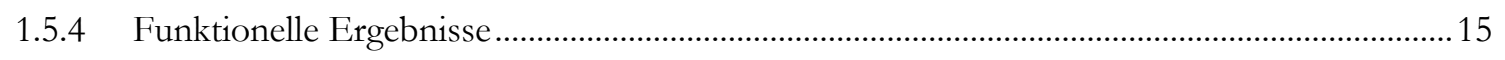

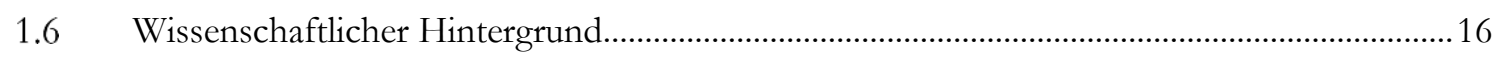

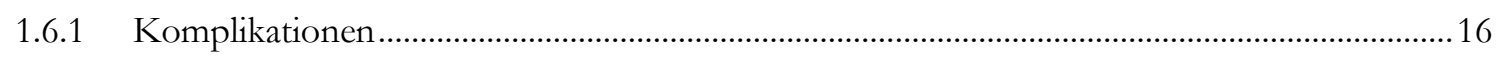

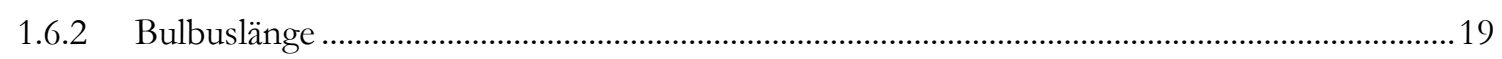

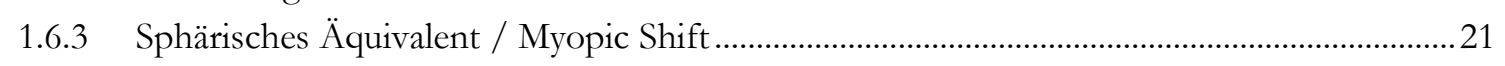

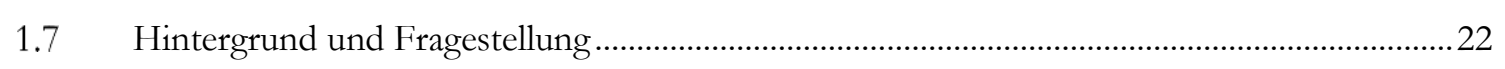

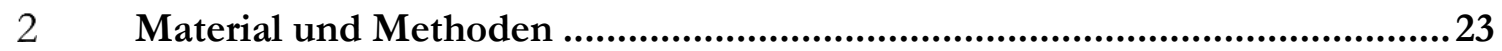

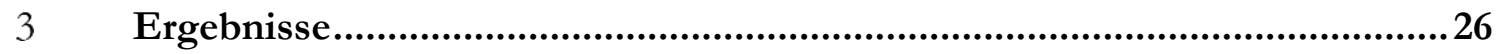

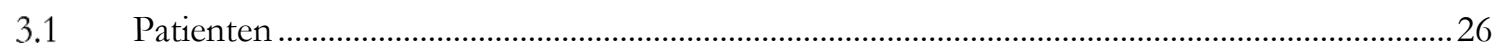

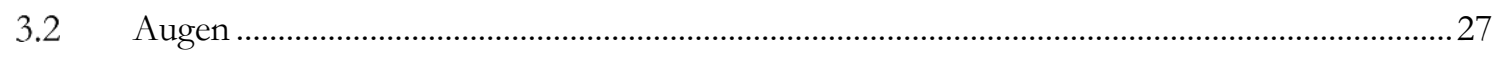

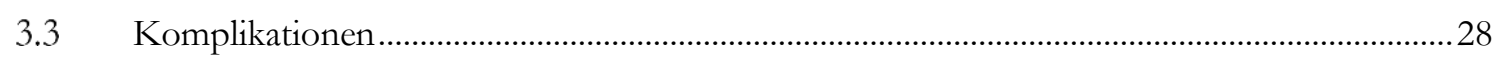

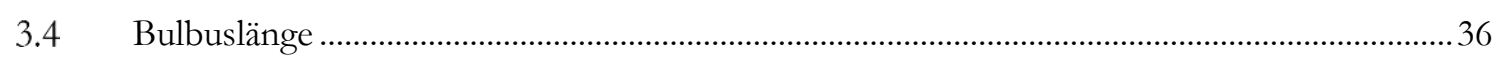

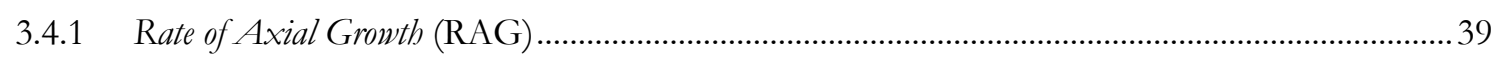

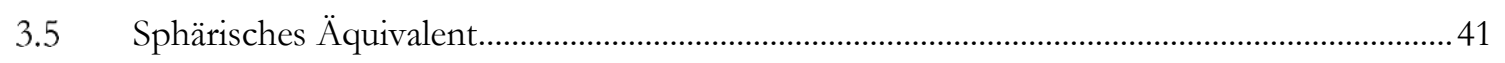

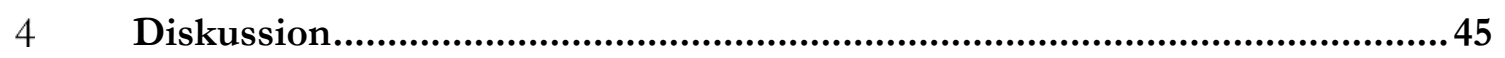

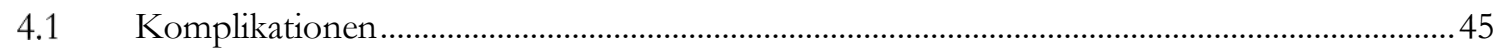

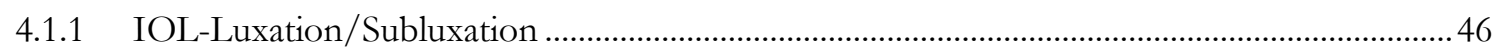

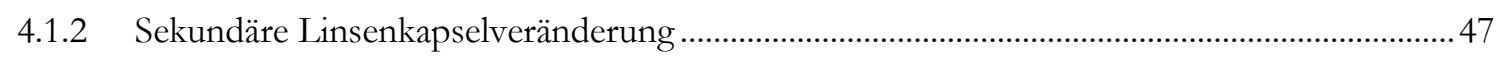

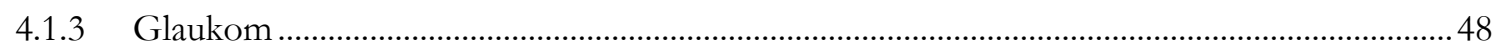

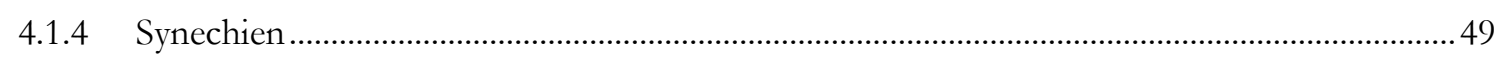

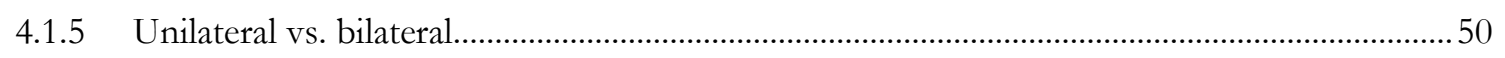




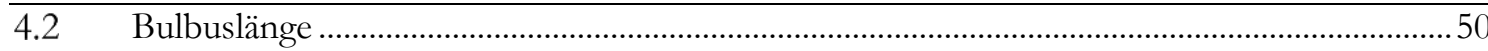

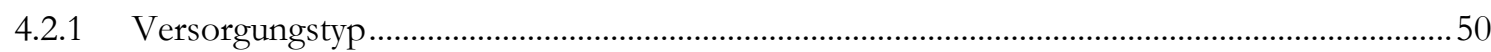

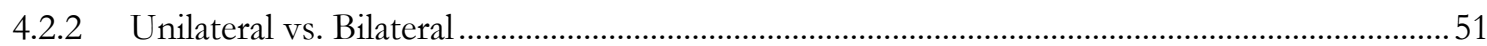

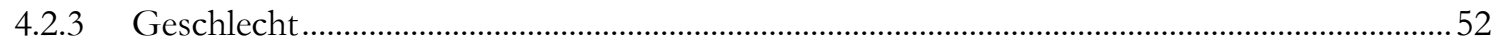

4.2.4 Patienten mit sekundärer Linsenkapselveränderung …........................................................5 52

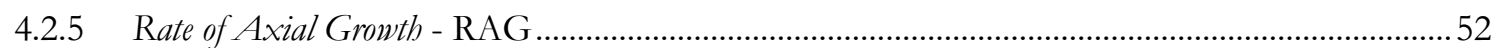

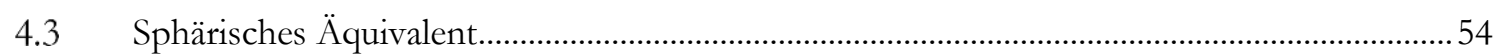

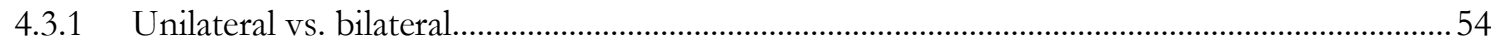

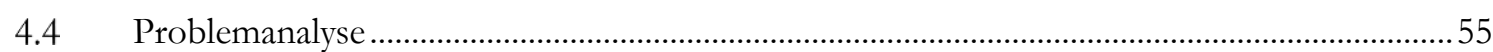

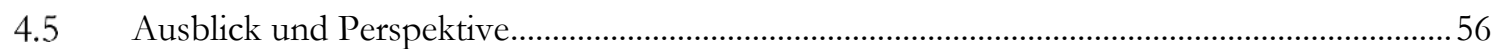

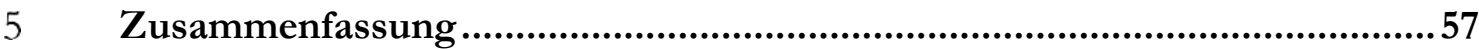

$6 \quad$ Literaturverzeichnis ......................................................................59 


\section{Abbildungsverzeichnis}

Abbildung 1 Linsenentwicklung

Abbildung 2 kleine (wahrscheinlich) nicht visus-relevante Kerntrübung .............................................. 4

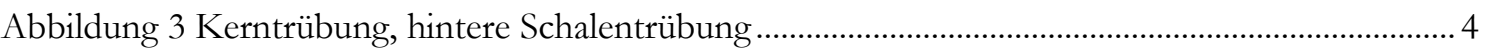

Abbildung 4 Limbaler Tunnel-Zugang bei primärer Implantation ............................................................ 8

Abbildung 5 vordere Kapsulorhexis bei primärer Implantation ......................................................... 9

Abbildung 6 Linsenmassenabsaugung bei primärer Implantation ........................................................ 9

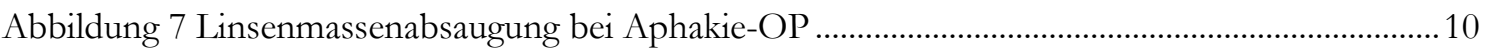

Abbildung 8 Eröffnung der vorderen und hinteren Kapsel mit vorderer Vitrektomie bei Aphakie-OP

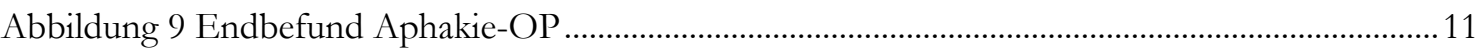

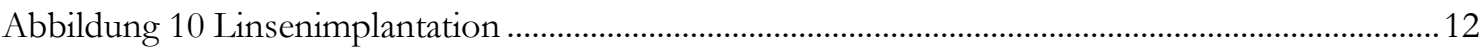

Abbildung 11 Hintere Kapsulorhexis und vordere Vitrektomie ........................................................12

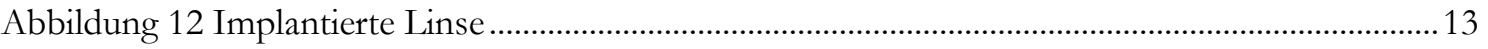

Abbildung 13 Anteil der Komplikationen an der Gesamtaugenzahl ...................................................22

Abbildung 14 Anteil der Komplikationen nach Versorgungstyp ........................................................29

Abbildung 15 Auftreten der Komplikationen nach Versorgungstyp........................................................ 30

Abbildung 16 Auftreten der Komplikationen nach Lateralität................................................................. 31

Abbildung 17 Prozentuale Verteilung bezüglich aller Komplikationen, der komplikationsbehafteten Augen und der von Komplikationen betroffenen Patienten..................................................... 31

Abbildung 18 Verteilung aller Komplikationen bezüglich des Versorgungstyps.....................................32

Abbildung 19 Verteilung aller komplikationsbehafteten Augen bezüglich des Versorgungstyps.......33

Abbildung 20 Verteilung aller von Komplikationen betroffenen Patienten bezüglich des Versorgungstyps.

Abbildung 21 Entwicklung der Bulbuslänge zwischen Baseline und letzter Messung nach Versorgungstyp

Abbildung 22 Verteilung des sphärischen Äquivalents bezügliche des Alters zum Messzeitpunkt nach Versorgungstyp

\section{Tabellenverzeichnis}

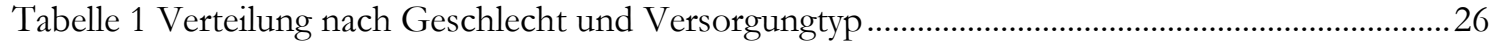

Tabelle 2 Verteilung der Patienten nach Lateralität und Versorgungstyp ……………...........................26

Tabelle 3 Durchschnittliche Nachuntersuchungsdauer in Jahren nach Versorgungstyp ....................27

Tabelle 4 Durchschnittliche Nachuntersuchungsdauer in Jahren nach Lateralität...............................27

Tabelle 5 Verteilung der Augen nach Lateralität und Versorgungstyp...................................................27

Tabelle 6 Alter bei Kataraktextraktion in Tagen nach Versorgungstyp .................................................28

Tabelle 7 Alter bei Kataraktextraktion in Tagen nach Lateralität...........................................................28

Tabelle 8 Verteilung der aus den Komplikationen resultierenden Re-Operationen nach

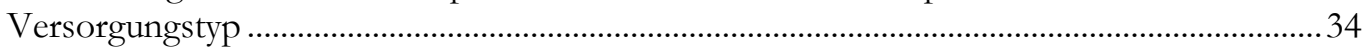

Tabelle 9 Patientenalter beim Auftreten der Komplikation in Jahren nach Versorgungstyp ..............34

Tabelle 10 Patientenalter beim Auftreten der Komplikation in Jahren nach Lateralität .......................34

Tabelle 11 Zeitraum Linsenimplantation bis Komplikation in Jahren nach Versorgungstyp .............35

Tabelle 12 Zeitraum Linsenimplantation bis Komplikation in Jahren nach Lateralität.........................35 
Tabelle 13 Verteilung der gesamten Komplikationen bei sekundär versorgten Patienten vor/nach Linsenimplantation

Tabelle 14 Verteilung der Augen mit Komplikationen bei sekundär versorgten Patienten vor/nach

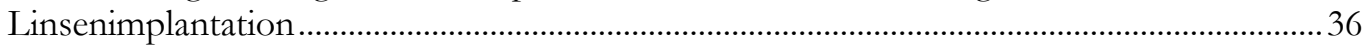

Tabelle 15 Durchschnittliche Bulbuslänge in $\mathrm{mm}$ je Zeitraum nach Versorgungstyp............................37

Tabelle 16 Durchschnittliche Bulbuslänge in mm je Zeitraum nach Lateralität...................................... 37

Tabelle 17 Durchschnittliche Bulbuslänge in mm je Zeitraum nach Lateralität.....................................38

Tabelle 18 Durchschnittliche Bulbuslänge in $\mathrm{mm}$ je Zeitraum bei Patienten mit der Komplikation sekundäre Linsenkapselveränderung nach Versorgungstyp ..................................................... 38

Tabelle 19 Durchschnittlicher Nachuntersuchungszeitraum in Tagen ..................................................39

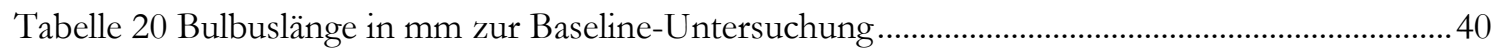

Tabelle 21 Bulbuslänge im mm zum Zeitpunkt der letzten Messung nach Versorgungstyp............... 40

Tabelle 22 Bulbuswachstum in mm nach Versorgungstyp...................................................................... 41

Tabelle 23 Rate of axial growth nach Versorgungstyp ................................................................................ 41

Tabelle 24 Zeitraum Kataraktextraktion bis letztmögliche Refraktionsmessung nach Versorgungstyp

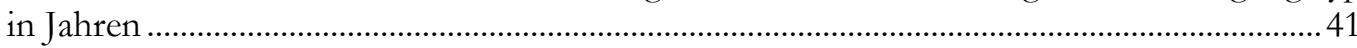

Tabelle 25 Zeitraum Kataraktextraktion bis letztmögliche Refraktionsmessung nach Lateralität in Jahren .42

Tabelle 26 Zeitraum Kataraktextraktion bis letztmögliche Refraktionsmessung nach Komplikation $\mathrm{ja} /$ nein in Jahren ............................................................................................................... 42

Tabelle 27 Durchschnittliches sphärisches Äquivalent in dpt je Zeitraum nach Versorgungstyp ..... 42

Tabelle 28 Durchschnittliches sphärisches Äquivalent in dpt je Zeitraum nach Lateralität . .43

Tabelle 29 Durchschnittliches sphärisches Äquivalent in dpt je Zeitraum nach Komplikation ja/nein 


\section{Abkürzungsverzeichnis}

$\mathrm{AL}$ Achslänge

CL Kontaktlinse

CPC Zyklophotokoagulation dpt ..Dioptrien IATS . Infant-Aphakia-Treatment-Study IOL Intraokularlinse IQR Interquartile range $\mathrm{Lj}$. Lebensjahr OR Odds Ratio OVD Ocular viscoelastic device RAG Rate of axial growth

RRG Rate of refractive growth TORCH(S) Toxoplasmosis, Other, Rubella, Cytomegalovirus, Herpes, (Syphilis) $\mathrm{VAO}$ Visual axis opacification YAG Neodym-dotierter Yttrium-Aluminium-Granat-Laser 


\section{$1 \quad$ Einleitung}

Die kindliche Katarakt entspricht mit einer weltweiten Prävalenz von schätzungsweise vier bis fünf Patienten pro 10.000 Kindern (Wu et al. 2016) nach europäischen Standards (<5/10 000 Kindern) definitionsgemäß einer seltenen Krankheit (Casteels-Rappagliosi 1999; Taruscio et al. 2011). Trotzdem ist die kindliche Katarakt die häufigste Ursache behandelbarer Blindheit im Kindesalter (Foster et al. 1997).

\section{1 Ätiologie}

Die kongenitale Katarakt definiert sich als eine bei Geburt vorhandene Linsentrübung. Diese kann in ihrer Vielzahl sowohl erblich als auch erworben sein.

Die Katarakt kann einseitig oder beidseitig auftreten. In der entwickelten Welt ist die Ursache der bilateralen kongenitalen Katarakt in den meisten Fällen idiopathisch. Ungefähr ein Drittel der Fälle sind hereditär, ohne Anteil einer systemischen Erkrankung (Merin und Crawford 1971; Bardelli et al. 1989).

Bei teils bekannten genetischen Dispositionen können kongenitale Katarakte trotz gleichen genetischen Stammes zu unterschiedlichen Ausprägungen führen. Diese Fälle sind in der Hauptsache autosomal dominant, treten jedoch auch autosomal rezessiv und x-gonosomal auf (Francis und Moore 2004; Hejtmancik 2008).

Eine bilaterale kongenitale Katarakt kann in Kombination mit systemischen Erkrankungen wie einer Trisomie 13, 18, und 21, dem Turner-, Lowe- oder Nance-Horan-Syndrom, der Galaktosämie oder dem Morbus Fabry auftreten (Nischal und Medsinge 2015). Dabei ist gewöhnlich eine geistige Retardierung mit der bilateralen kongenitalen Katarakt verbunden. Dazu trifft bei vielen erworbenen Erkrankungen diese Kombination mit weiteren Veränderungen zusammen, wie zum Beispiel: kranionfacialen und skelettalen Deformitäten, Myopathie, Spastik oder anderen neurologischen Störungen (Zetterström et al. 2005). Fortwährend werden immer neue Gene entdeckt die einen Einfluss auf die Entstehung der kongenitalen Katarakt haben (Francis und Moore 2004; Hejtmancik 2008; Churchill und Graw 2011).

Diverse intrauterine Infektionen haben auf die Kataraktentwicklung einen prägenden Einfluss. Insbesondere die häufigsten Infektionen der TORCH(S)-Gruppe (Toxoplasmose, Herpes, Syphilis, Röteln, Cytomegalie) sowie die Varizellen-Infektionen können die kongenitale Katarakt auslösen (Lambert et al. 1989b; Nischal und Medsinge 2015). Davon ist die Röteln-Infektion die wichtigste.

Eine unilaterale kongenitale Katarakt ist vornehmlich nicht mit systemischen Krankheiten assoziiert und selten erblich bedingt (Lim et al. 2010). In einigen Fällen besteht eine Verbindung zum Lentikonus/Lentiglobus oder zu einer persistierenden Arteria hyaloidea (Amaya et al. 2003; 
Lüchtenberg und Kohnen 2007). In diesen Fällen ist es wichtig, die Defekte frühzeitig zu erkennen, um Schwierigkeiten und Komplikationen während einer eventuellen Operation zu entgehen. Traumatische Ursachen sollten bei einer kongenitalen unilateralen Katarakt in jedem Fall ausgeschlossen werden (Nischal und Medsinge 2015).

\subsection{Morphologie}

\subsubsection{Embryonale Linsenentwicklung}

Das Auge entwickelt sich zuerst aus dem Neuroektoderm des Vorderhirns, welches im Verlauf mit verschiedenen Anteilen der Augenanlage in Wechselwirkung tritt. Dabei entsteht aus dem Neuroektoderm des Vorderhirns die Retina, die Schichten des Ziliarkörpers, die hinteren Schichten der Iris und der Nervus opticus. Das Kopfmesenchym, zwischen dem Neuroektoderm des Vorderhirns und dem Oberflächenektoderm liegend, bildet Sklera, Kornea und Choroidea. Aus dem Oberflächenektoderm des Kopfes entstehen Augenlinse und das Kornealepithel.

Bald nachdem sich am 28. Tag aus dem Neuroektoderm die Augenbläschen oder sog. Sehventrikel ausgebildet haben, kommen diese, sich nach distal ausdehnend, mit dem Oberflächenektoderm des Kopfes in Kontakt. An der Kontaktstelle verdickt sich das Oberfächenektoderm und die Linsenanlage bildet sich als sog. Linsenplakoid aus. Das Linsenplakoid senkt sich ein und es entsteht eine Linsengrube. Gleichzeitig mit dem Formen des Augenbechers durch das Neuroektoderm nähern sich die Ränder des Linsenplakoids an, verlieren ihren Kontakt zum Oberflächenektoderm und es entsteht das Linsenbläschen.

Am Linsenbläschen unterscheidet sich ein vorderer und ein hinterer Pol sowie die dazwischenliegende Äquatorialzone. Die Zellen der Hinterwand verlängern sich und durchsetzen schrittweise vollständig das Lumen. Sie verlieren Ihre Kerne und bilden lichtdurchlässige Kristalline. Es entstehen die transparenten primären Linsenfasern. Die Zellen der Äquatorialzone, welche sich ebenfalls verlängern und ihre Kerne verlieren, lagern sich den primären Linsenfasern als sekundäre Linsenfasern an und vergrößern damit bis ins Erwachsenenalter hinein den Linsendurchmesser. Die Linsenkapsel, als Entsprechung der Basalmembran des Linsenepithels, verdickt sich und dient sowohl der Stabilität der Linse als auch als Ansatz der Zonulafasern (siehe Abbildung 1).

Die Versorgung der Linse erfolgt innerhalb der ersten fünf Monate über die Glaskörperarterie sowie die Tunica vasculosa lentis. Danach wird die Linse nur noch durch Diffusion aus dem Kammerwasser und dem Glaskörper versorgt. (Moore und Persaud 2007) 


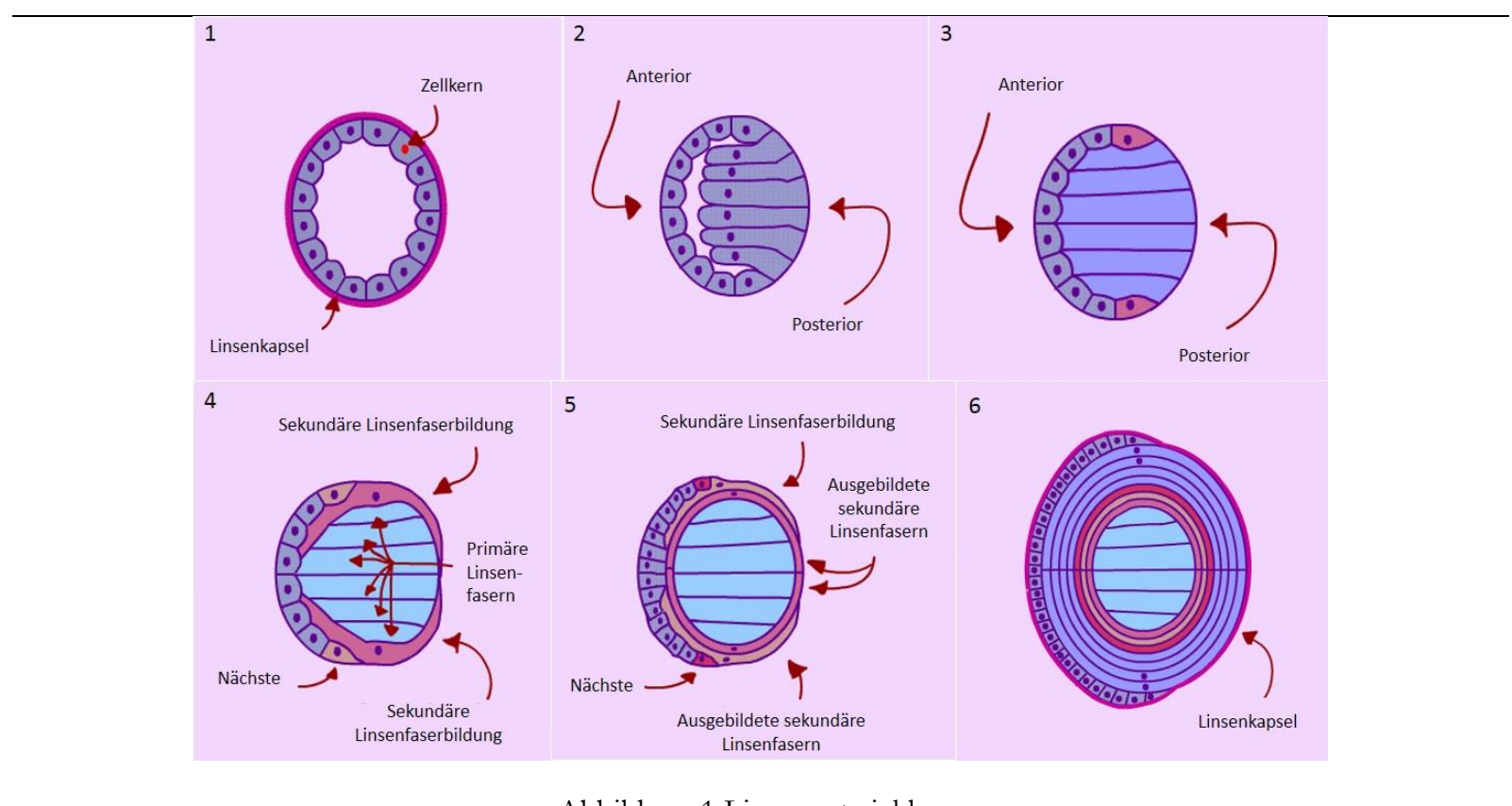

Abbildung 1 Linsenentwicklung

(Von Ventus55 - Eigenes Werk, CC BY-SA 3.0, https://commons.wikimedia.org/w/index.php?curid=29883487)

\subsubsection{Kataraktformen}

Eine detaillierte Beschreibung der Morphologie der kindlichen Katarakt hilft nicht nur bei der Ermittlung spezifischer Ursachen, sondern auch im Rahmen der konservativen und chirurgischen Therapieplanung (Nischal und Medsinge 2015).

\section{- Kernkatarakt}

Die Kernkatarakt ist gewöhnlich schon bei Geburt vorhanden und nicht progressiv (Parks et al. 1993). Die Trübung beschränkt sich auf den embryonalen und fetalen Nukleus und ist im Zentrum gewöhnlich sehr dicht (siehe Abbildung 2 und 3).

Die Augen sind meist kleiner als normal. Tierversuche zeigen, dass bei frühzeitig lentektomierten Tieren das Wachstum auch bei gutem retinalen Bild reduziert ist (Kugelberg et al. 1996).

In $80 \%$ der Fälle ist die Katarakt bilateral. Hier kann in $30-50 \%$ eine Vererbung nachgewiesen werden, die generell autosomal-dominant ist (Lüchtenberg und Kohnen 2007). 


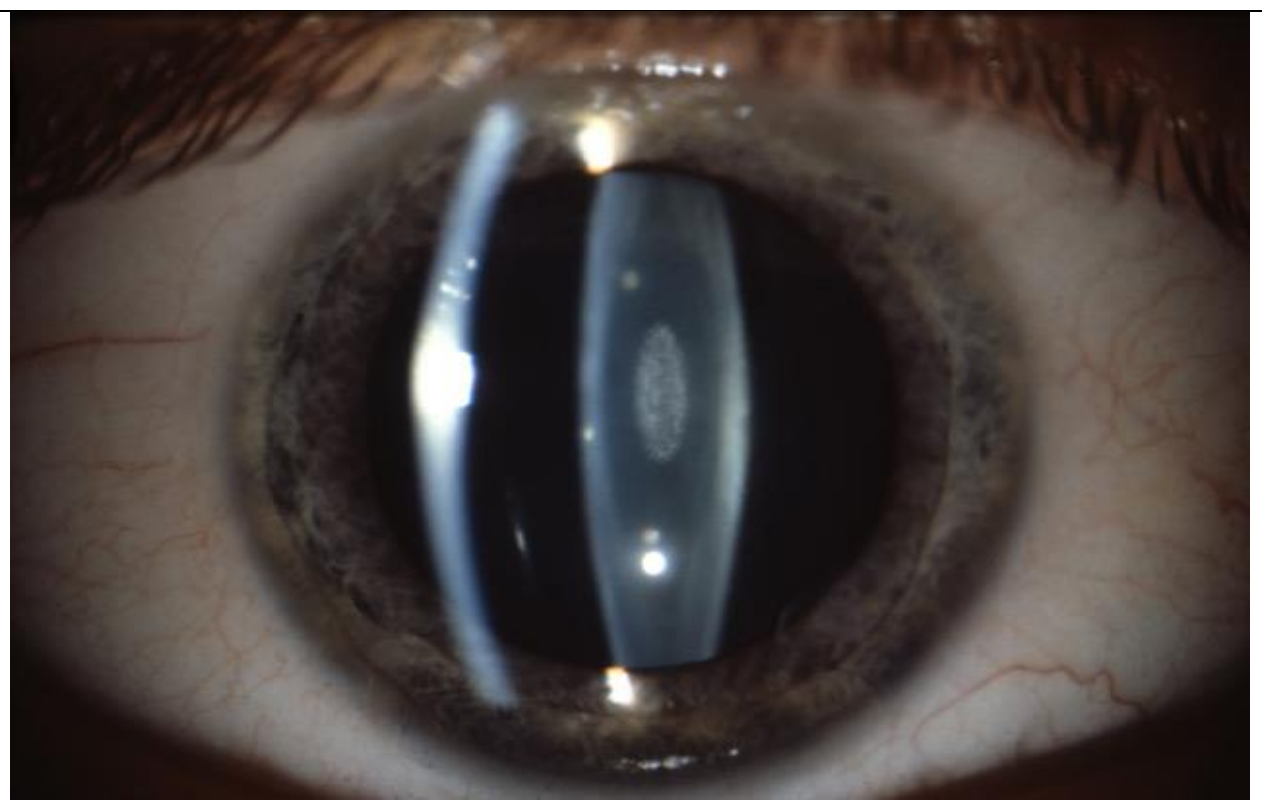

Abbildung 2 kleine (wahrscheinlich) nicht visus-relevante Kerntrübung (mit freundlicher Genehmigung Dr. med. J. Callizo)

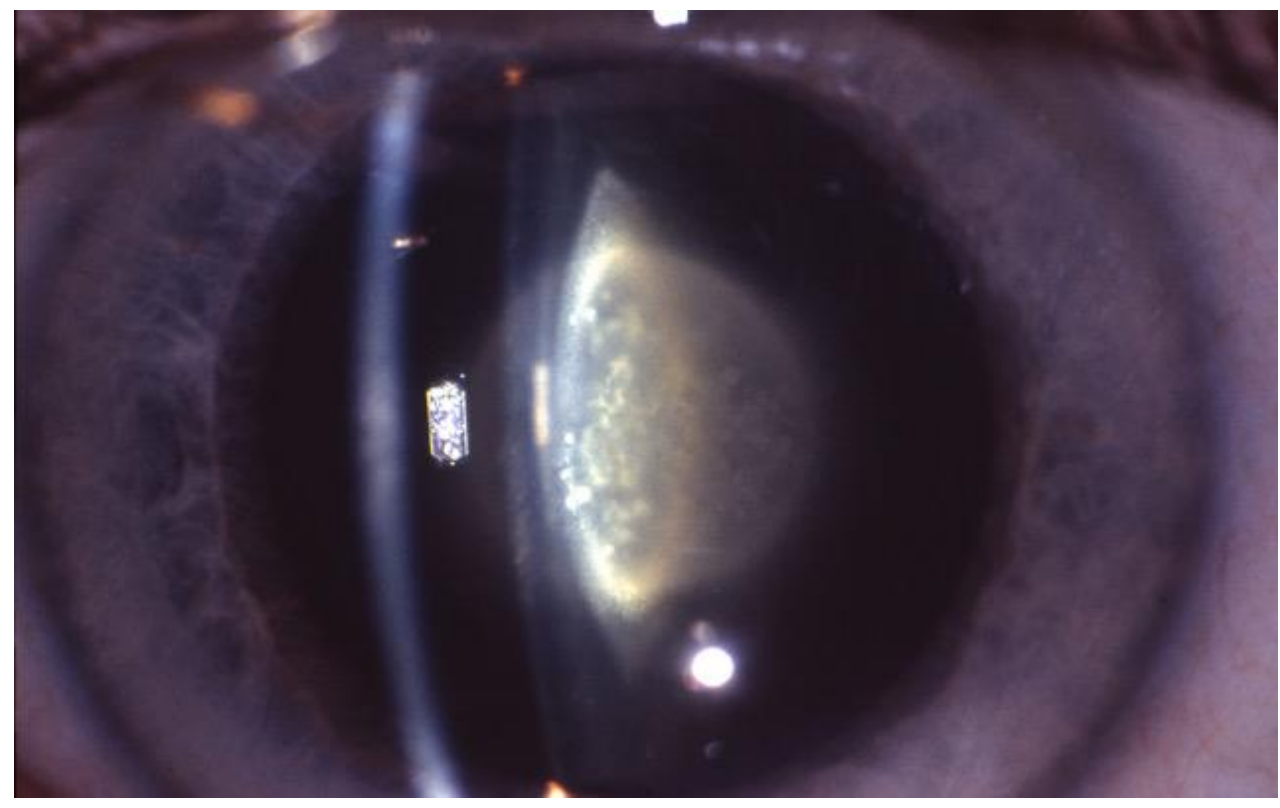

Abbildung 3 Kerntrübung, hintere Schalentrübung (mit freundlicher Genehmigung von Dr. med. J. Callizo)

\section{- Posteriore Katarakt}

Die einseitige posteriore Katarakt bei Kindern ist oft mit einer persistierenden Arteria hyaloidea oder einem persistierenden hyperplastischen Glaskörper assoziiert. Beim betroffenen Auge besteht gewöhnlich ein Mikrophthalmus (Zetterström et al. 2005).

Der Kontakt zwischen den retrolentalen Strukturen und der Kapsel ermöglicht das Umschlingen der Linse mit Blutgefäßen, was insbesondere intraoperativ zu Blutungen führen kann. Daher empfiehlt sich besonders bei reduziertem Einblick, präoperativ eine Ultraschalluntersuchung 
durchzuführen. Der fibrovaskuläre Stiel kann außerdem zu einer traktiven Ablatio retinae führen. Das Sekundärglaukom stellt eine häufige Komplikation nach früher operativer Versorgung dieser Augen dar (Lundvall und Kugelberg 2002a). Trotzdem ist bei fehlendem Einblick und der Hoffnung auf Sehverbesserung einer Linsenoperation indiziert.

\section{- Lamelläre Katarakt}

Die lamelläre Katarakt findet sich zwischen dem klaren fetalen Kern und der klaren Rinde (Parks et al. 1993). Sie entwickelt sich in der Regel, nachdem sich die Fixation etabliert hat, und ist gewöhnlich progressiv. Die Augen sind zumeist von normaler Größe mit normal großer Kornea. Die lamelläre Katarakt ist immer beidseitig und in der Mehrzahl autosomal dominant vererbt.

\section{- Andere Kataraktformen}

Andere Kataraktformen stehen in der Regel mit Linsenentwicklungsdefekten in Verbindung und sind bei Geburt vorhanden. Dazu gehören z. B. Trübungen am Embryonalkern und der vordere Polstar. Diese zeigen meist keinen Progress und führen nicht zwangsläufig zu einer Visusreduktion.

\subsection{Amblyopie und kongenitale Katarakt}

„Amblyopie (syn. entwicklungsbedingte Sehschwäche) ist das Ergebnis einer gestörten zentralnervösen visuellen Verarbeitung, wobei die neuronalen Voraussetzungen für deren Entwicklung normal sind oder es einmal waren. Leitsymptom ist die verminderte bestkorrigierte Sehschärfe insbesondere für Reihenoptotypen. Amblyopie entsteht durch partielle oder vollständige Reizdeprivation und/oder eine Störung der binokularen Zusammenarbeit" (DOG/BVA - Leitlinie Nr. 26a 2010 zu Amblyopie, S. 2)

Die Amblyopie ist wahrscheinlich verursacht durch die strukturelle und funktionelle Fehlentwicklung des lateralen Corpus geniculatum und des Cortex striatum durch nicht ausreichende visuelle Stimulation während der sensiblen Entwicklungsperiode des visuellen Systems (Wiesel und Hubel 1963). Diese Periode liegt schwerpunktmäßig in den ersten drei bis sechs Lebensmonaten, dauert jedoch insgesamt länger (Lüchtenberg und Kohnen 2007). Andere Untersuchungen sehen die kritische Phase gar in den ersten sechs Lebenswochen (Elston und Timms 1992). Die Anfälligkeit für die Amblyopie sinkt dann schrittweise bis zum Alter von sechs bis sieben Jahren, wenn die Reifung des visuellen Systems abgeschlossen ist und die visuellen Zentren sowie der retinokortikale Pfad unempfindlich gegen abnormale Sehempfindungen geworden sind (van Noorden 1978).

Bei Kindern mit kongenitaler Katarakt ist daher wegen der vom ersten Lebenstag an bestehenden getrübten Sehachse das Risiko der Amblyopie-Entstehung von besonderer Bedeutung.

Die Umkehrbarkeit der Amblyopie hängt vom Entwicklungsstand des visuellen Systems zum Zeitpunkt der Entstehung der Deprivation und vom Lebensalter zum Therapiebeginn ab. Das impliziert einen frühen Therapiebeginn einer dichten kongenitalen Katarakt, um eine irreversible Amblyopie und einen eventuellen Nystagmus zu verhindern (Taylor et al. 1979; Rogers et al. 1981; 
Dutton 1990; Kugelberg 1992). Der Sehverlust ist außerdem von der Dichte, der Größe und der Lokalisation der Katarakt abhängig. So können Kinder mit teilweiser subklinischer Katarakt eine normale Entwicklung des visuellen Systems aufweisen (Zetterström et al. 2005).

Eine unilaterale Katarakt ist immer mit einer schlechteren Visusprognose vergesellschaftet. Hier wirkt neben der Deprivation zusätzlich die Suppression durch das gesunde Auge amblyogen. Neben einer frühzeitigen Okklusionstherapie ist daher eine engmaschige klinische Evaluation sowohl der monokularen als auch der binokularen Fixationsmuster vonnöten (Birch et al. 1998).

\subsection{Präoperative Untersuchungen}

Eine sorgfältige präoperative Untersuchung der betroffenen Kinder ist Bestandteil jeder Diagnostik Je zentraler und je weiter posterior die Katarakt liegt, desto größer ist der Einfluss auf das visuelle System (Lambert et al. 1989a).

Erste Auffälligkeiten wie eine ungewöhnliche Kopfzwangshaltung, Schielen, Blendempfindlichkeit oder eine mangelnde Fixation oder Blickkontaktaufnahme können Eltern auf eine mögliche Trübung aufmerksam machen. Kinder mit klinisch relevanter bilateraler Katarakt erscheinen in ihrer neurologischen Entwicklung verzögert und fallen durch ihr beeinträchtigtes Sehen auf. Im Gegensatz dazu wird eine monokulare Katarakt bei Kindern fast immer später entdeckt, da ihre Sehfähigkeit oft nicht offensichtlich beeinträchtigt scheint, diese Kinder fallen häufig erst durch Strabismus auf. Die Schielstellung entwickelt sich jedoch in der Regel erst dann, wenn bereits ein irreparabler Sehverlust besteht. Das Auftreten eines manifesten Nystagmus im Alter von weniger als zwei Monaten weist gewöhnlich auf eine schlechte Prognose der Sehfähigkeit hin (Rogers et al. 1981; Kugelberg 1992; Parks et al. 1993). Bleibt das Kind im Alltag unauffällig, ist die U2-U6Untersuchung des Kinderarztes von essentieller Bedeutung, um Anomalien frühzeitig zu erkennen.

Der Brückner-Test, die gleichzeitige Beleuchtung beider Augen mit einem Ophthalmoskop mit Bewertung des roten Fundusreflexes, ist ein probates Mittel zur schnellen Einschätzung des Lichtweges des Auges (Brückner 1965). Ein Unterschied im Fundusreflex kann auf eine Verlegung hinweisen. Dabei lässt sich besonders bei enger Pupille die Zentralität gut bewerten. Eine zentrale Trübung von mehr als $3 \mathrm{~mm}$ verlangt aufgrund ihrer Relevanz in der Regel nach chirurgischer Entfernung. Auffälligkeiten sollten immer zu einer Abklärung führen (Gräf 2007).

Die Skiaskopie ermöglicht neben dem Ausschluss einer Ametropie den Ausgleich einer Fehlsichtigkeit, was besonders bei geringgradigen Trübungen zu einer Re-Evaluation der OPIndikationen führen kann.

Mit der Spaltlampe können auch bei Kleinkindern Größe, Ort und Dichte der Trübung sowie kapsuläre Veränderungen, wie zum Beispiel vorbestehende Defekte der posterioren Kapsel, ermittelt werden. Im diesem Zuge sollte auch die Messung des Augeninnendruckes und des Durchmessers der Kornea erfolgen (Nischal und Medsinge 2015). 
Wenn möglich, sollte eine Visusprüfung mit einem altersentsprechenden Mittel (Teller Acuity Cards, Cardiff Test usw.) erfolgen. Ein Visus von $<0,4$ bei Kindern im Alter von drei Jahren lässt die Erwägung einer Operation zu (Lüchtenberg und Kohnen 2007).

Eine direkte Ophtalmoskopie kann ebenso zur Zusammenschau der Befunde beitragen. Ist die Makula bei Miosis nicht einsehbar, kann dies zur operativen Indikationsstellung beitragen.

Die B-Bild-Untersuchung im Ultraschall lässt Hinweise auf Anomalien des hinteren Augenabschnittes zu. Dies gilt insbesondere, wenn dieser zum Beispiel aufgrund dichter Trübungen nicht anders einsehbar ist. Eine A-Bild-Messung der Achsenlänge dient nicht nur dem Nachweis eines Mikrophthalmus und der Einordnung hinsichtlich der Wachstumstendenz, sondern auch der Berechnung der passenden Linse bei primärer Implantation einer intraokularen Linse (IOL). Sollten Alter des Kindes und die Dichte der Linsentrübung es erlauben, empfiehlt sich der Versuch, eine Non-Contact-Coherence-Interferometrie (z. B. mittels IOL-Master der Fa Zeiss) durchzuführen (Findl et al. 2001).

Die vollständige Untersuchung von Säuglingen benötigt oft eine Sedierung oder eine Vollnarkose. Dabei sollten beide Augen untersucht werden, da auch am nicht durch Katarakt betroffenen Auge häufig Fehlbildungen festzustellen sind (Zetterström et al. 2005). Obwohl Hinweise bestehen, dass die am wachen Kind ermittelten Parameter äquivalent zu den unter Narkose ermittelten sind (Kinori et al. 2015), empfehlen aktuelle Übersichten noch immer die Untersuchung in Narkose (Lim et al. 2017).

Die Indikation zur Operation sollte erst nach Zusammenschau aller Befunde, nicht jedoch aufgrund einzelner Testergebnisse getroffen werden (Lüchtenberg und Kohnen 2007).

Des Weiteren empfiehlt es sich insbesondere bei der bilateralen Katarakt, Blut-, Urin- oder Kapselgewebeproben einzusenden, um zum Beispiel eine Galaktosämie oder ein Lowe-Syndrom auszuschließen (Lüchtenberg und Kohnen 2007).

\subsection{Chirurgische Therapie}

Die chirurgische Therapie der kindlichen Katarakt ist mit der Operation bei Erwachsenen kaum vergleichbar. Dies begründet sich nicht nur in der Größe des kindlichen Auges, sondern auch in dessen weicher und elastischer Gewebestruktur und der verstärkten Entzündungsreaktion auf das chirurgische Trauma (Kohnen und Lüchtenberg 2007). Daneben muss das Kind länger als der Erwachsenen mit dem Ergebnis der Behandlung leben. Daher muss das Risiko der AmblyopieEntstehung ohne operative Behandlung besonders berücksichtigt werden.

\subsubsection{Lentektomie}

In Anbetracht der frühen sensiblen Entwicklungsperiode des visuellen Systems (Kapitel 1.3) hat sich gezeigt, dass bei der unilateralen kindlichen Katarakt die Kataraktextraktion (Lentektomie) innerhalb der ersten vier bis sechs Wochen und bei der bilateralen Katarakt innerhalb der ersten 
sechs bis acht Wochen die Entwicklung von Amblyopie, Strabismus und Nystagmus verhindern kann (Birch und Stager 1996; Birch et al. 2009).

Es stehen grundsätzlich mehrere Zugangswege zur Linse zur Verfügung (siehe Abbildung 4). Der sklerale Pars-plana/Pars-plicata-Zugang und der sklerokorneale bzw. korneale Zugang durch die Vorderkammer. Die Wahl des Zugangs ist abhängig von Prägung und Erfahrung des Operateurs, wobei bis dato wenige Untersuchungen zu Vor- und Nachteilen veröffentlicht sind (Ahmadieh et al. 1999; Bar-Sela und Spierer 2005; Lüchtenberg et al. 2008). Lagrèze tendiert in seiner Übersichtsarbeit (Lagrèze 2009) aufgrund der besseren Erreichbarkeit der Kapsel, der Undichtigkeit von limbalen Zugängen, ästhetisch auffälligen Bindehautvernarbungen sowie von möglicherweise Astigmatismus verursachenden Hornhautnähten zum skleralen Zugang. Theoretische Nachteile dieses Verfahrens sind die potentielle Zonulolyse, die im Verlauf des Lebens zu einer Instabilität des Kapselapparates und eventuellen Linsenluxation führen kann. Zudem besteht die Gefahr der Netzhautablösung durch Glaskörperreizung, was in dem kindlichen Alter eine verheerende Komplikation darstellt.

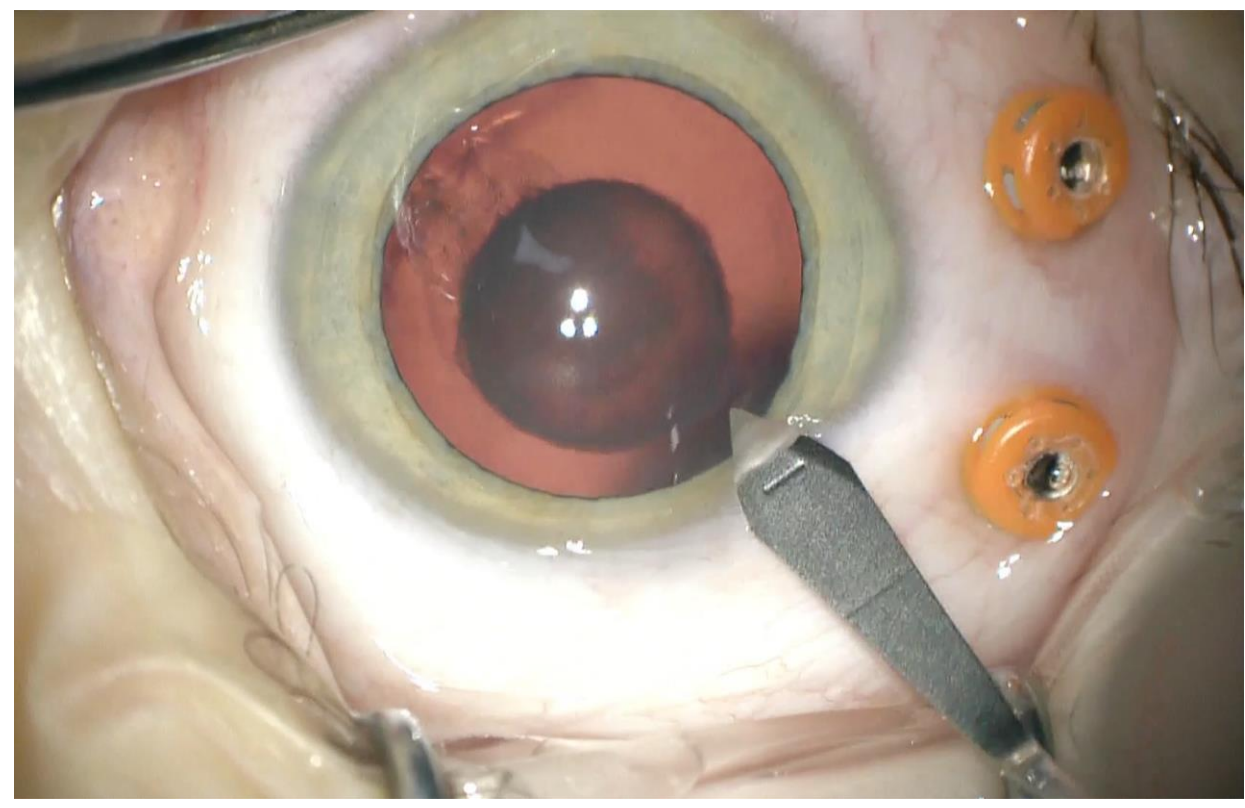

Abbildung 4 Limbaler Tunnel-Zugang bei primärer Implantation (mit freundlicher Genehmigung von Prof. Dr. med. W. A. Lagèze)

Wird der sklerokorneale Zugang über die Vorderkammer gewählt, empfiehlt es sich, die Vorderkammer stabil zu halten. Dies geschieht entweder durch eine separate Vorderkammerinfusion oder durch ein Viskoelastikum mit hohem Molekulargewicht (OVD: ocular viscoelastic device, z. B. Healon GV).

Die Mehrheit der Operateure in Deutschland gibt an, die periphere Kapsel zu erhalten (Lagrèze 2009). Dies geschieht vor allem wegen einer einfacheren sekundären IOL-Implantation, wodurch eine sklerafixierte Linseneinnähung vermieden werden kann. Das teilweise Auftreten von hinteren Synechien aus zurückgebliebenem Linsenepithel und die dadurch erschwerte sekundäre Implantation wird dabei in Anbetracht der Vorteile in Kauf genommen. 
Der Operateur wählt ein oder zwei Zugänge (skleral oder korneal) und legt einen Infusionszulauf. Es folgt die Eröffnung der vorderen Kapsel (Kapsulorhexis) mit dem Vitrektom, manuell oder neuerdings versuchsweise mit dem Femtosekundenlaser (siehe Abbildung 5). Daraufhin kommt es zur Entfernung der Linse (i. d. R. durch Absaugen) (Abbildung 6 und 7).

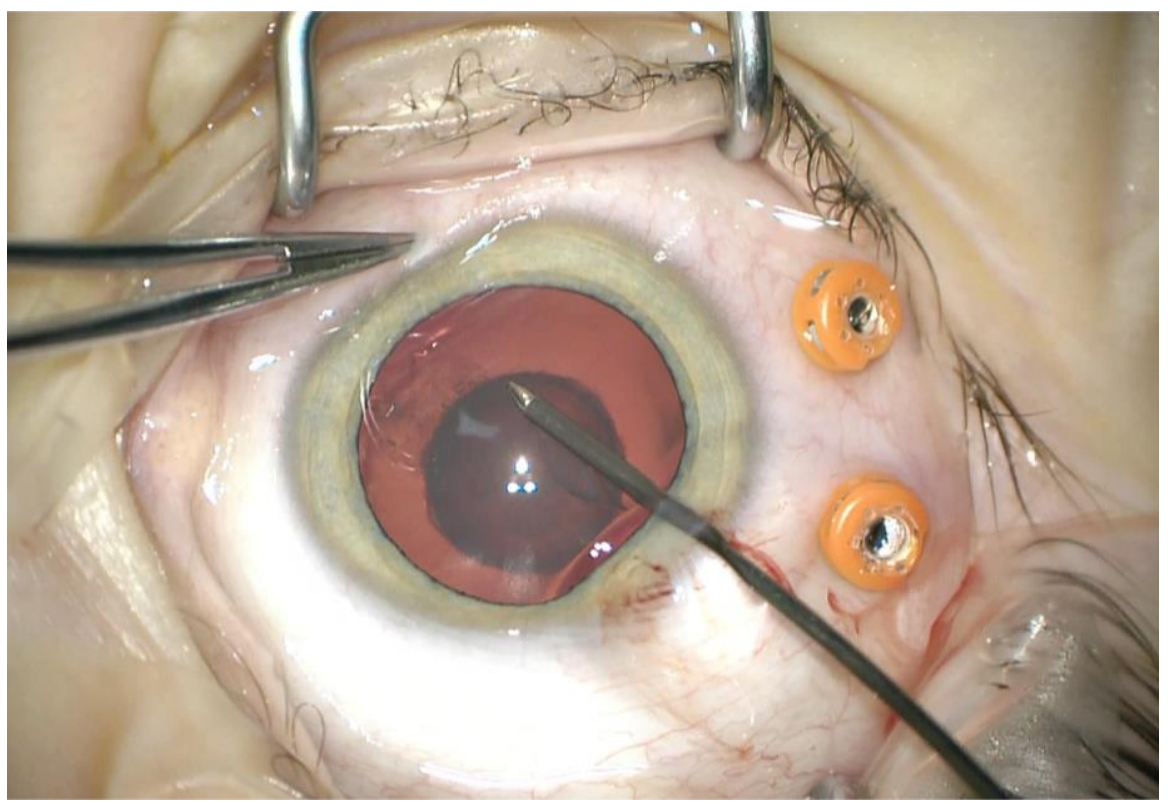

Abbildung 5 vordere Kapsulorhexis bei primärer Implantation (mit freundlicher Genehmigung von Prof. Dr. med. W. A. Lagrèze)

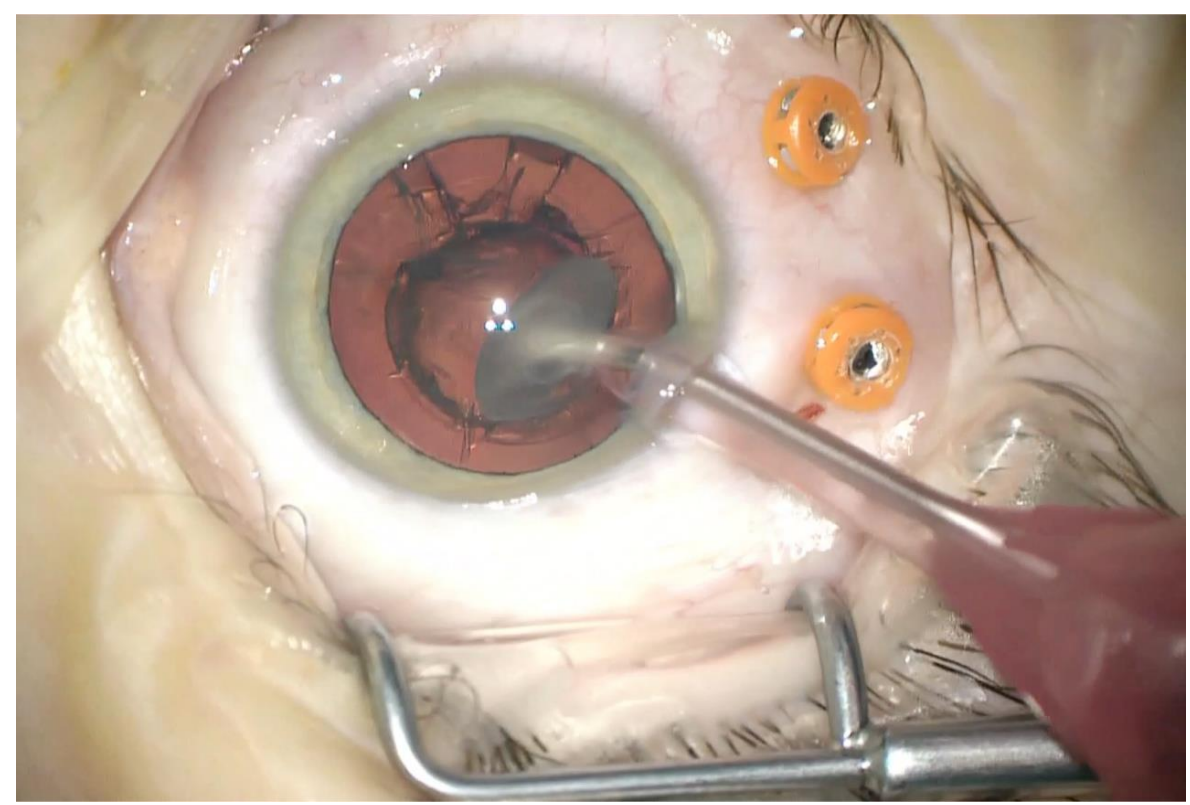

Abbildung 6 Linsenmassenabsaugung bei primärer Implantation (mit freundlicher Genehmigung von Prof. Dr. med. W. A. Lagrèze) 


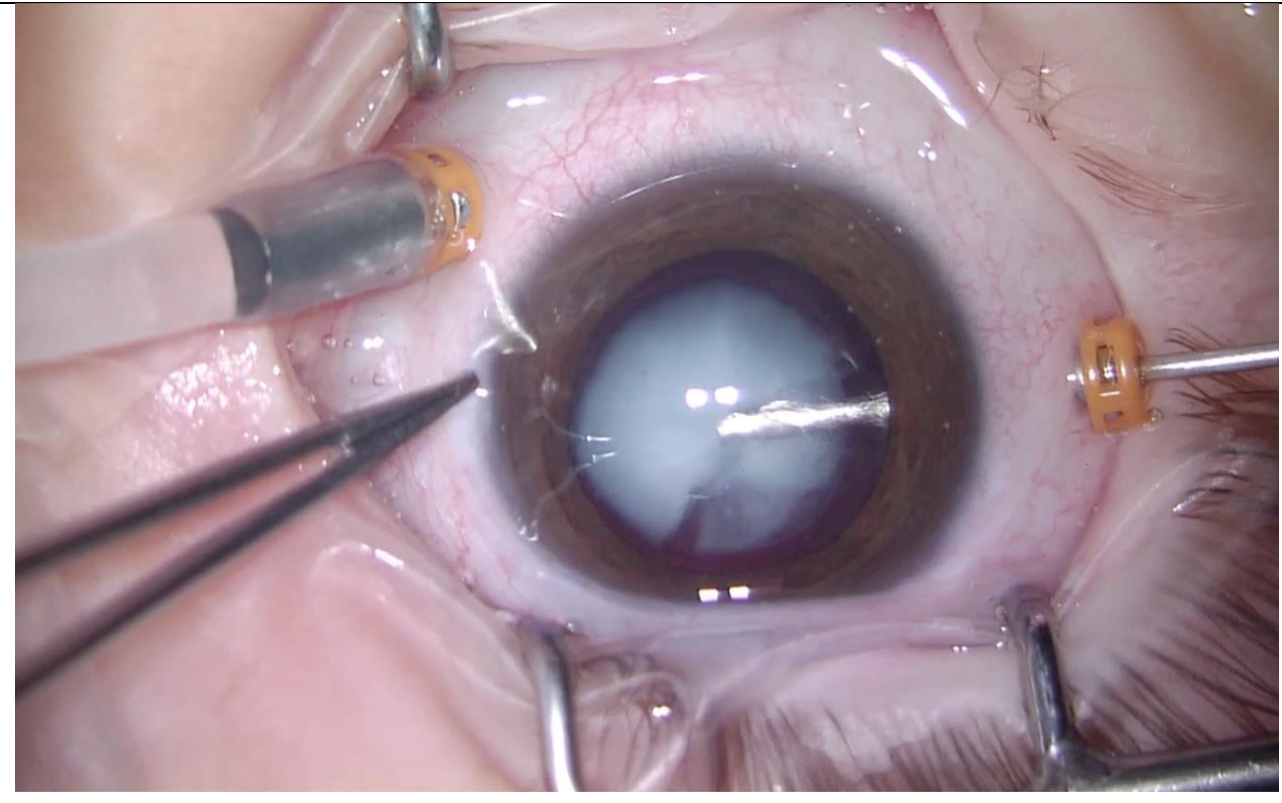

Abbildung 7 Linsenmassenabsaugung bei Aphakie-OP (mit freundlicher Genehmigung von Prof. Dr. med. W. A. Lagrèze)

Es folgen die Politur der vorderen und hinteren Kapsel sowie die Eröffnung der hinteren Kapsel mit der Vitrektom. Verbleibt die hintere Kapsel, entwickeln 100\% der Augen mit weniger als vier Lebensjahren signifikante sekundäre Linsenkapselveränderungen (Stager et al. 2002). Nun werden die Kapselblätter weiter eröffnet. Dabei ist besonders darauf zu achten, dass kein Linsenmaterial in den Glaskörperraum absinkt. Da der vordere Glaskörper als Gerüst für die Proliferation von Zellen des Linsenepithels dient (Kugelberg und Zetterström 2002), werden die vorderen Glaskörperanteile ausgeschnitten (vordere Vitrektomie) (siehe Abbildung 8) und es erfolgt gegebenenfalls der Wundverschluss (Abbildung 9).

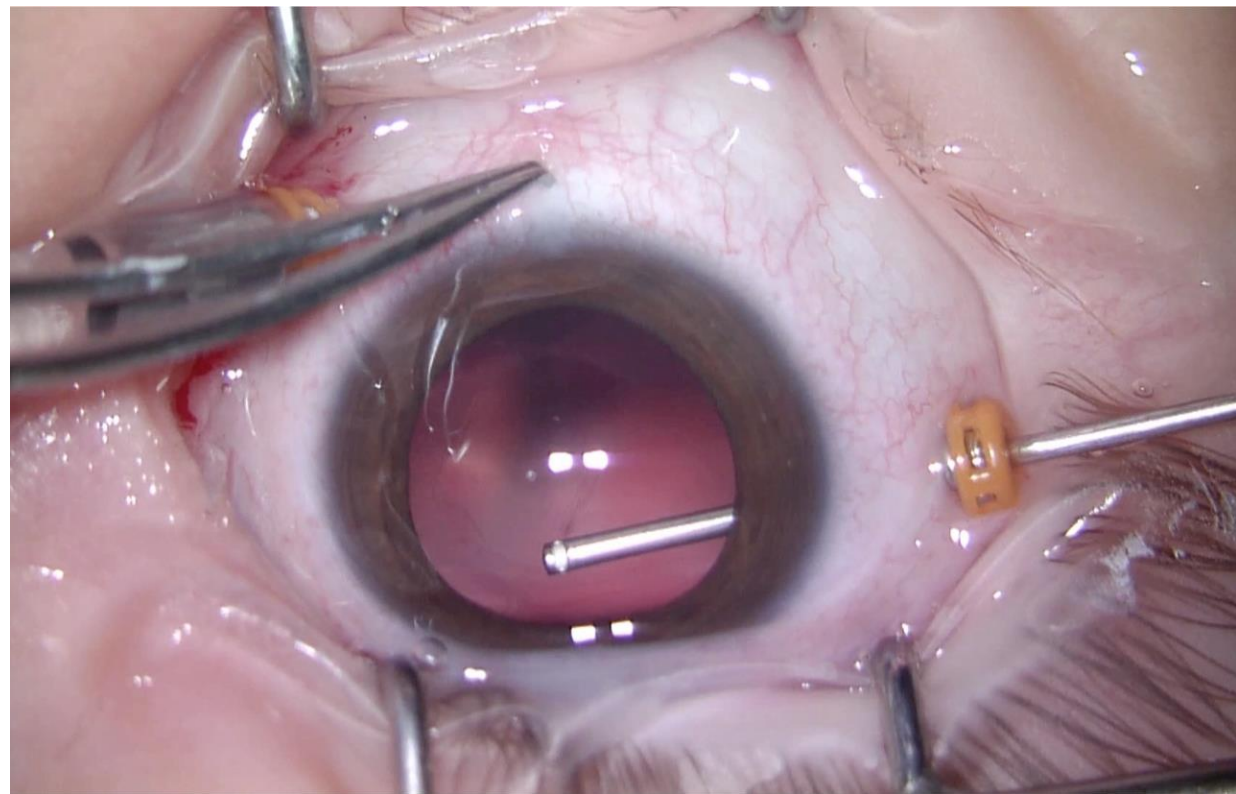

Abbildung 8 Eröffnung der vorderen und hinteren Kapsel mit vorderer Vitrektomie bei Aphakie-OP (mit freundlicher Genehmigung von Prof. Dr. med. W. A. Lagrèze) 


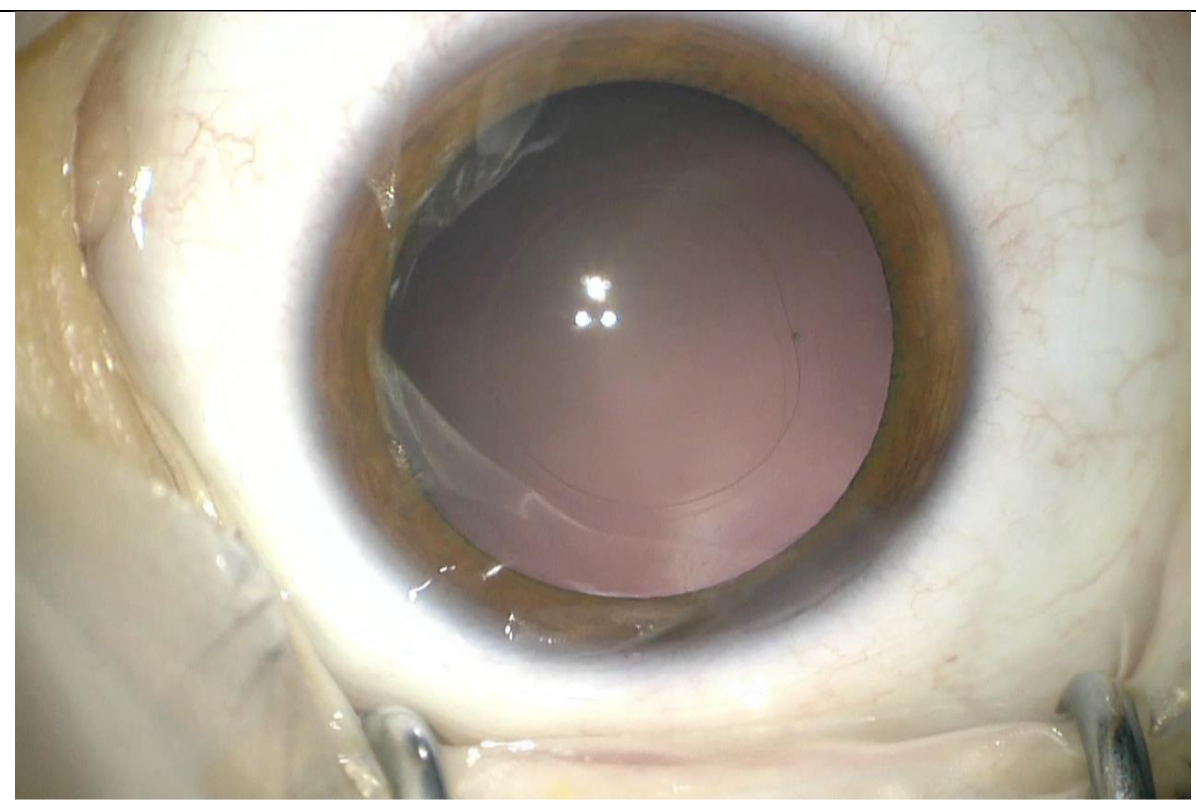

Abbildung 9 Endbefund Aphakie-OP

(mit freundlicher Genehmigung von Prof. Dr. med. W. A. Lagrèze)

Bis vor einigen Jahren wurde die Operation standardmäßig mit einem 20-Gauge-Vitrektom durchgeführt. Bei Kindern, jünger als sechs Monate, kann dies jedoch zu Problemen wie Trübungen der Kornea an der Insertionsstelle, unbeabsichtigten Kapselverletzungen, Iris-Prolaps und Glaskörperprolaps führen. Außerdem können bei größeren Schnitten Leckagen entstehen, welche Nähte benötigen, in deren Folge es zu Astigmatismus kommen kann. Die Leckagen können bis zu acht Wochen Heilungszeit benötigen (Raina et al. 2016). Fortschritte in der Instrumentenentwicklung ermöglichen heute kleinere Schnitte mit bis zu 27-Gauge (entspricht 0,4 mm Außendurchmesser). Kleinere Wunden ermöglichen eine größere Stabilität der vorderen Kammer sowie ein nahtloses Operationsverfahren. Dagegen steht ein erhöhtes Infektionsrisiko wie beim Erwachsen (Dave et al. 2016). Bezüglich 25-Gauge-Systemen wurde anfangs eine zu große Flexibilität der Geräte und daraus entstehende Probleme sowie Schwierigkeiten bei der Aspiration und eine verlängerte Operationszeit berichtet (Chee und Lam 2009; Raina et al. 2016). Mittlerweile wurden die Probleme allerdings gelöst und die Instrumente sind stabil.

Eine topische Medikation mit Steroiden und Antibiotika nach der Operation wird empfohlen, um die sofort einsetzende Entzündungsreaktion in der postoperativen Periode zu unterdrücken (Cleary et al. 2010; Mataftsi et al. 2012). Diese wird in den ersten Tagen stündlich appliziert und dann über drei bis sechs Wochen ausgeschlichen.

\subsubsection{Intraokularlinsen}

Die grundlegenden chirurgischen Schritte der Intraokularlinsenimplantation haben sich in den letzten Jahren nicht maßgeblich verändert. Verschiedene Techniken unterscheiden sich vor allem durch den Zugangsweg und die Reihenfolge der Operationsschritte (Implantation, hintere Kapsulorhexis, vordere Vitrektomie). Laut einer Umfrage ist die häufigste in Deutschland 
durchgeführte Technik eine hintere Kapsulorhexis mit anschließender vorderer Vitrektomie und nachfolgender IOL-Implantation über einen kornealen oder korneoskleralen Zugang (Noack und Lagrèze 2008) (Abbildung 10, 11 und 12).

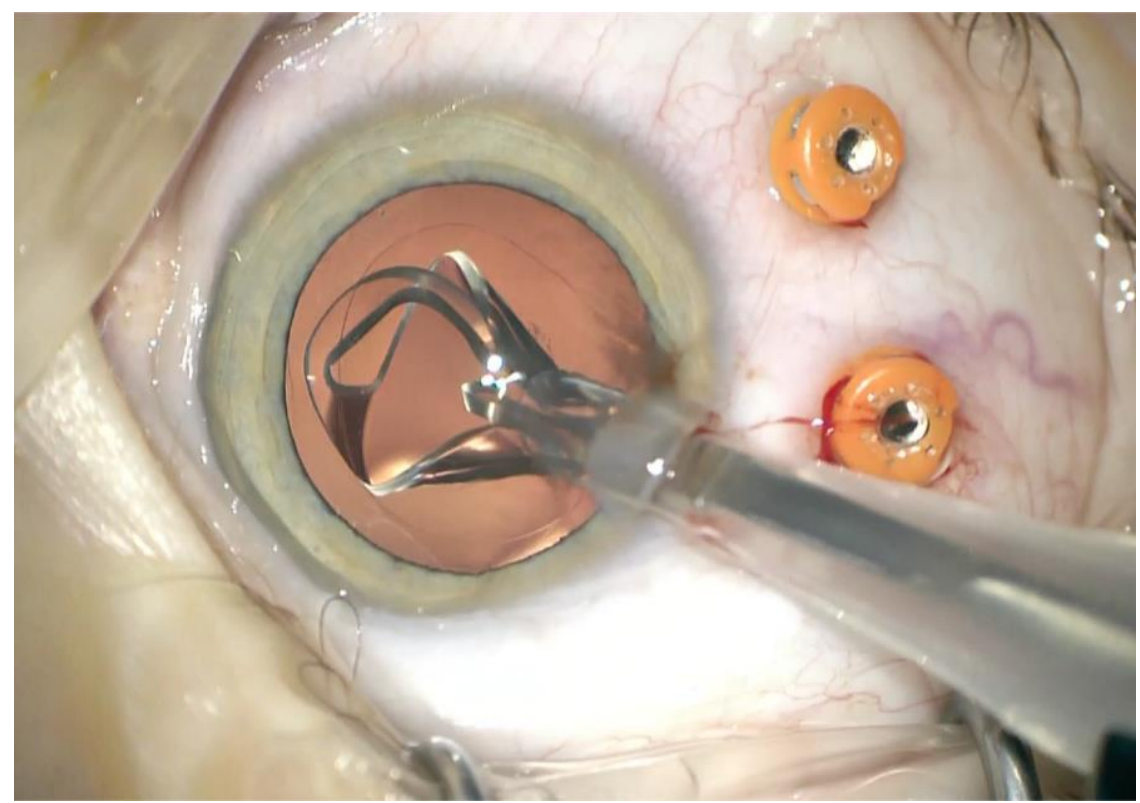

Abbildung 10 Linsenimplantation (mit freundlicher Genehmigung von Prof. Dr. med. W. A. Lagrèze)

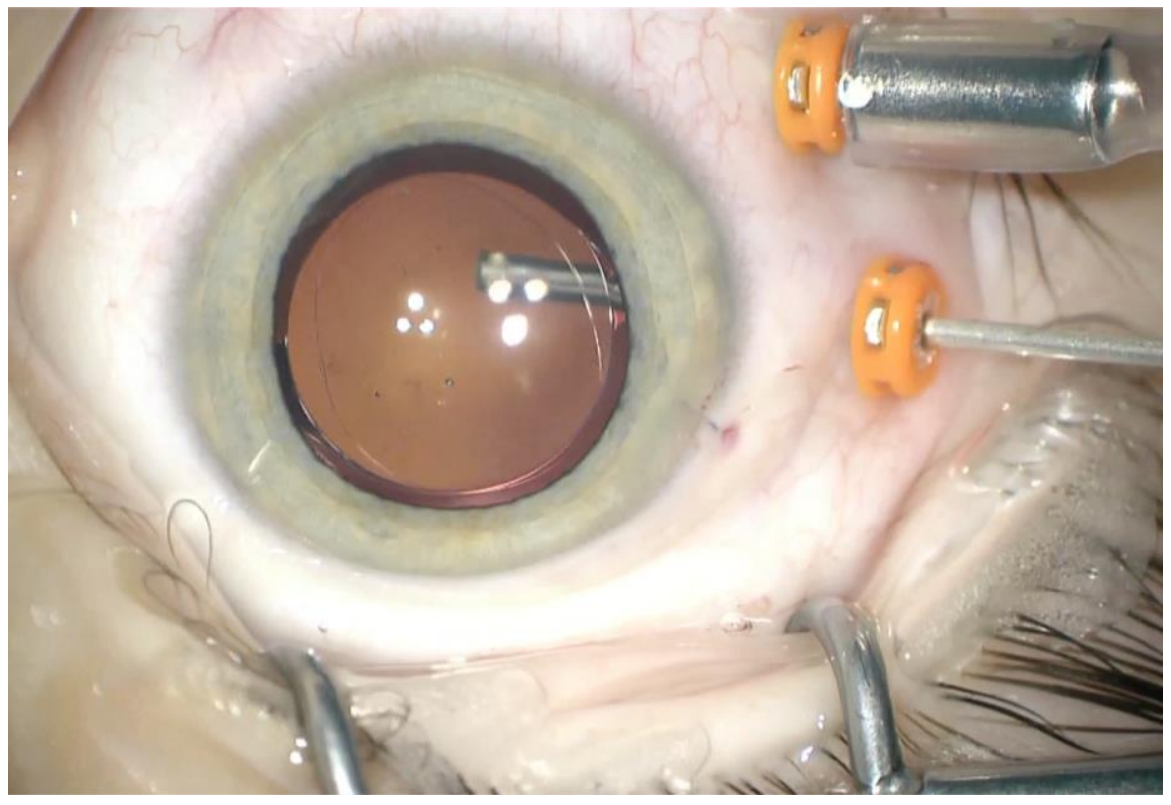

Abbildung 11 Hintere Kapsulorhexis und vordere Vitrektomie (mit freundlicher Genehmigung von Prof. Dr. med. W. A. Lagrèze) 


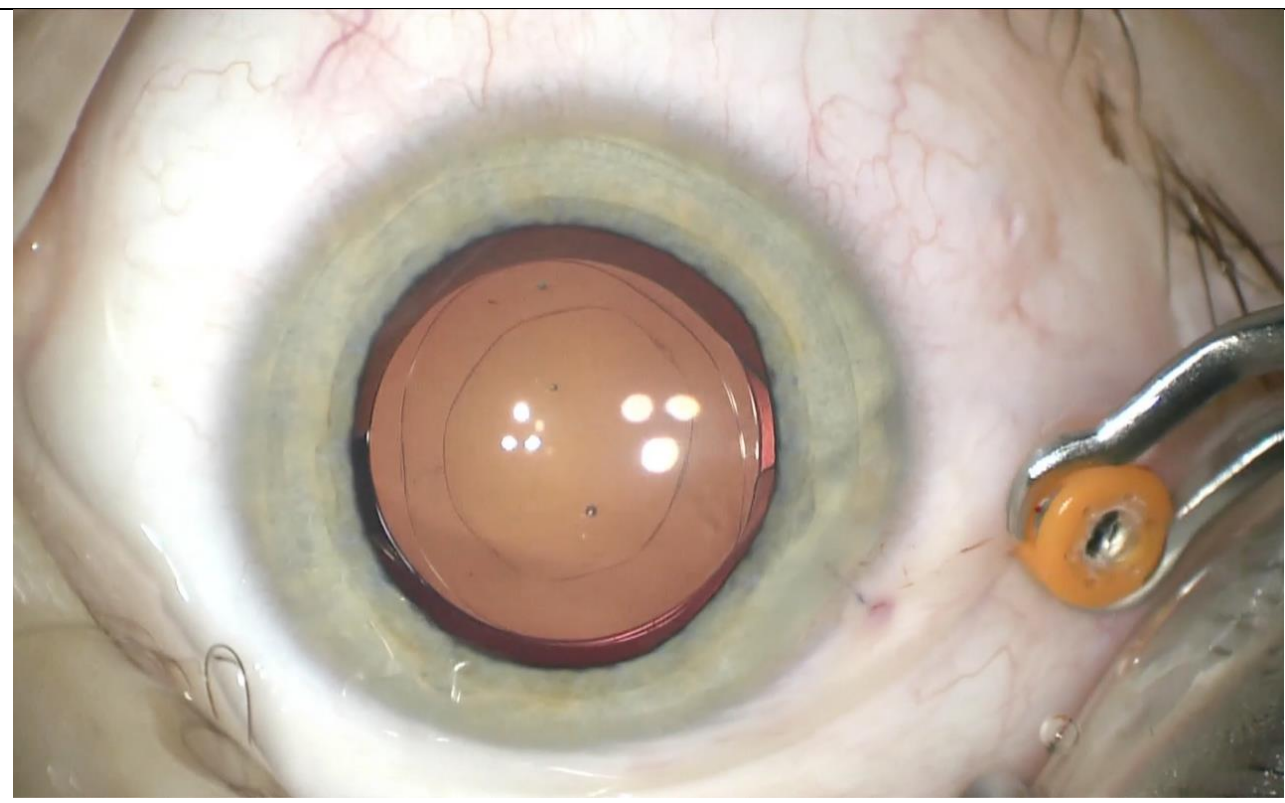

Abbildung 12 Implantierte Linse

(mit freundlicher Genehmigung von Prof. Dr. med. W. A. Lagrèze)

Die Wahl der Intraokularlinse gestaltet sich bei Kindern aufgrund verschiedenster Faktoren kompliziert. Dazu gehören zum Beispiel anormales Wachstums des Auges, schlechte Vorhersagbarkeit durch Linsen-Formeln besonders bei kleinen Augen mit normalem Wachstum, Ungenauigkeiten der Messung der Achsenlänge und der Keratometrie, verstärkte korneosklerale Elastizität bei Kindern und Schwierigkeit der Ermittlung der nötigen Parameter beim wachen Kind.

Das Bulbuswachstum und die daraus zu erwartende Myopisierung verlangen nach einer Hyperopisierung des zu operierenden Auges. Dabei gilt: je kleiner das Kind, desto stärker die Hyperopisierung. Der mittlere Myopic Shift sinkt vom zweiten auf das achte Lebensjahr von ca. 6 dpt auf ca. 2 dpt (Crouch et al. 2002). Der Grad der Hyperopisierung sollte außerdem abhängig von der Refraktion der Gegenseite gemacht werden (Lim et al. 2017). Die Sanders-Retzlaff-Kraff (SRK)/theroretical-(T)-, Holladay-I- und SRK-II-Formeln bieten dabei die verlässlichsten Ergebnisse bei kleinen kindlichen Augen (VanderVeen et al. 2013; Joshi et al. 2014). Außerdem hängt die Wahl der Zielrefraktion vom Aufmerksamkeitsradius des Kindes ab.

Bei historisch gewachsenen unterschiedlichen Linsentypen und -materialien hat sich zu diesem Zeitpunkt unter den Chirurgen die faltbare hydrophobe einteilige Acryllinse zur Implantation in den Kapselsack oder die dreiteilige Linse zur Implantation in den Sulcus ciliaris durchgesetzt (Wilson und Trivedi 2007; Lagrèze 2009; Lim et al. 2017). Einen weiteren positiven Effekt auf das Ergebnis bietet die Technik des optic capture bei dem die Linse durch die hintere Kapselöffnung geflochten wird, so dass die Kapselblätter verschmelzen können (Zhou und Zhou 2016). Die neuerdings in Europa vermarktete bag-in-lens (BIL) ermöglicht über zwei elliptisch geformte Haptiken, dass sich die Kapselblätter ähnlich dem optic capture verkleben. Die Optik bietet dabei eine physische Barriere und zeigt vielversprechende Ergebnisse bezüglich der Entwicklung sekundärer Linsenkapselveränderungen (Morcher 2016), wobei die Elastizität der Kapsel und die präzise Kapsulorhexis, welche zur Positionierung der Linse benötigt wird, einen Einfluss auf das 
Ergebnis haben (Van Looveren et al. 2015). Weitere Langzeitstudien müssen diese Ergebnisse jedoch noch bestätigen. Auch multifokale Linsen werden teils mit ermutigenden Ergebnissen verwendet, sind jedoch wegen refraktiver Ungenauigkeit infolge des Myoptic Shifts bei Kindern nur als „off-label“ zugelassen (Sadoughi et al. 2015; Lapid-Gortzak et al. 2016). VorderkammerIntraokularlinsen und Iris-fixierte Intraokularlinsen zeigen Probleme wie endothelialen Zellverlust, Linsendislokation und Glaukom-Entwicklung, während Sklera-fixierte Linsen in 12-24\% ein Nahtbruch und eine Dislokation aufweisen (Jacobi et al. 2002; Asadi und Kheirkhah 2008; Buckley 2008). Diese drei Linsentypen sollten bei Kindern mit Vorsicht verwendet werden (Nischal und Medsinge 2015; Lim et al. 2017).

Als Zeitpunkt der Implantation hat sich als standard of care (Lambert 2016) ein Kindesalter von frühestens zwei Jahren durchgesetzt. Vorher ist die Kontaktlinsenversorgung Therapie der Wahl. Ausnahme bilden patientenspezifischen Umstände wie z. B. eine geringe Compliance bei der korrekten Durchführung der Kontaktlinsenversorgung (Lim et al. 2017). Trotzdem wurde auch von guten Ergebnissen bei primärer IOL-Implantation bei Kindern unter zwei Jahren berichtet (Sukhija et al. 2014).

\subsubsection{Komplikationen}

Die Trübung der visuellen Achse (VAO: visual axis opacification) ist die am weitesten verbreitete Komplikation nach Kataraktchirurgie am Kind (Plager et al. 2014; Whitman und Vanderveen 2014), obwohl Techniken wie die Eröffnung der Hinterkapsel, die vordere Vitrektomie, die Verwendung von hydrophoben Acryllinsen, die selten verwandte Technik des optic capture und neuerdings die Implantation der bag-in-lens die Trübungsrate reduziert haben. Wenn eine VAO auftritt, kann bei Erwachsenen im ersten Therapieversuch eine Neodynum YAG (Nd:Yag)Laserkapsulotomie durchgeführt werden. Diese ist jedoch häufig inadäquat, bei kleinen Kindern oft nicht möglich und es zeigt schon wenige Monate später ein Rezidiv (O’Keefe et al. 2001). Die chirurgische Intervention in Vollnarkose ist daher oft notwendig. Dabei wird in der Regel über einen limbalen Schnitt die Vorderkammer mit einer Infusion oder mit einem OVD stabilisiert, die Membran entfernt und der Eingriff durch eine vordere Vitrektomie ergänzt. Das OVD wird entfernt und der Zugang mit einer Naht verschlossen. Danach folgt in der Regel eine topische Steroidtherapie für einige Wochen.

Das Sekundärglaukom ist eine weitere Komplikation der kindlichen Kataraktchirurgie. Das Glaukom nach operativer Therapie der kongenitalen Katarakt ist dabei schwer zu handhaben und verlangt meist mehrere Behandlungsschritte (Bayoumi 2015). Einige Augen mit Sekundärglaukom können mit topischer Medikation kontrolliert werden. In vielen Fällen jedoch ist eine filtrierende Operation (z. B. Trabekulektomie mit Mitomycin C), ein zyklodestruktives Verfahren oder gar eine Shuntanlage erforderlich. Während ein geringes Alter zum Zeitpunkt der kindlichen Kataraktchirurgie mit einem besseren visuellen Ergebnis verbunden ist, steigt mit niedrigem Kindesalter das Risiko zur Glaukom-Entwicklung (Hartmann et al. 2015; Lambert 2016). Augen mit nuklearer Katarakt, kleiner Kornea oder persistierender Arteria hyaloidea sind mit einem 
höheren Glaukom-Risiko assoziiert (Parks et al. 1993; Freedman et al. 2015). Studien der InfantAphakia-Treatment-Study (IATS), einer prospektiv randomisierten multizentrischen Studie, weisen darauf hin, dass die Implantation einer Intraokularlinse nicht, wie lange gedacht, vor der Entwicklung eines Sekundärglaukoms schützt (Lambert et al. 2014; Freedman et al. 2015). Die lebenslange Kontrolle des Augeninnendrucks und des optischen Nervs ist ein wichtiger Stützpfeiler in der Nachsorge nach kindlicher Kataraktchirurgie (Zetterström et al. 2005).

Die verstärkte postoperative Entzündungsreaktion bei Kindern kann zu fibrinoiden Reaktionen, Dezentralisation der Intraokularlinse, Pigmentablagerungen auf der Intraokularlinse und posterioren Synechien führen (Nischal und Medsinge 2015). Die Ausbildung einer posterioren Synechie ist besonders bei Neugeborenen ohne Linsenimplantation häufig (Zetterström et al. 2005).

Postoperativer Strabismus tritt bei 55-100\% der Patienten mit kongenitaler Katarakt, insbesondere unilateraler kongenitaler Katarakt auf (Bothun et al. 2013; Magli et al. 2017). Die Infant-AphakiaTreatment-Study (IATS) zeigte, dass es keinen Unterschied zwischen den Gruppen gab (primäre IOL-Implantation vs. aphak mit Kontaktlinse). Bei Kindern die zum Zeitpunkt der Lentektomie älter waren ( $\geq 49$ Tage ), trat der postoperative Strabismus jedoch signifikant häufiger auf als bei den jüngeren Kindern (29 aus 50 Kindern vs. 51 aus 64 Kindern). (Bothun et al. 2013).

In der Untersuchung von unerwünschten Ereignissen und Nachoperationen ermittelt die IATS eine signifikant höhere Rate zusätzlicher chirurgischer Interventionen und signifikant mehr intraoperative Komplikationen in der IOL-Gruppe verglichen mit der Kontaktlinsengruppe, erstrangig wegen VAO und zweitrangig Glaukom bedingt (Lambert et al. 2014; Plager et al. 2014; Whitman und Vanderveen 2014; Solebo et al. 2015). Faktoren, wie zum Beispiel Unterschiede im peri-operativen Steroidregime oder die Erfahrung des Chirurgen, hatten keinen Effekt auf die unerwünschten entzündlichen Reaktionen (Lambert et al. 2015).

\subsubsection{Funktionelle Ergebnisse}

Das Ergebnis der Kataraktchirurgie hängt von diversen Faktoren ab, wie dem Alter des Beginns, der Ein- oder Beidseitigkeit, vom Typ, von vorexistierenden Deformitäten oder zusätzlichen Erkrankungen des Auges, Komplikationen nach der Operation oder vom Erfolg der AmblyopieTherapie zum Beispiel durch Okklusionsbehandlung. Das Hauptaugenmerk liegt daher darin, das Ergebnis der Sehfähigkeit zu verbessern und dabei das Risiko für Komplikationen zu minimieren. Kinder mit bilateraler Katarakt, die aphak belassen werden, kommen in der Regel mit einer Brillenoder Kontaktlinsenversorgung gut zurecht (Kumar und Lambert 2016). Bei unilateralen Katarakten stellt sich der Umgang mit postoperativer Anisometrie und Amblyopie als herausfordernd dar. Trotzdem zeigen sich sowohl bei uni- als auch bei bilateraler Katarakt gute visuelle Ergebnisse nach primärer IOL-Implantation (Struck 2015; Magli et al. 2016b; Magli et al. 2016a). Die IATS zeigte gleichzeitig mit der IoLunder2-Studie, dass bei unilateraler Katarakt die primäre Linsenimplantation die Wahrscheinlichkeit für bessere visuelle Ergebnisse nicht erhöht (Lambert et al. 2014; Solebo et al. 2015). Bei bilateraler Katarakt zeigen sich jedoch in der IoLunder2-Studie 
Hinweise für bessere visuelle Ergebnisses bei IOL-Implantation. Eine Abwägung der Vorteile einer primären Implantation gegen die höhere Wahrscheinlichkeit perioperativer Komplikationen insbesondere bei der unilateralen Katarakt bleibt also essentiell.

\subsection{Wissenschaftlicher Hintergrund}

Da es sich bei der kongenitalen Katarakt um eine seltene Erkrankung unterschiedlichster Ursachen handelt ist es schwer, aus der vorhandenen Literatur allgemeingültige Schlüsse zu ziehen. Die vorliegende Untersuchung bezieht sich insbesondere auf Kinder, die im ersten Lebensjahr operiert wurden, eine Gruppe mit speziellen Herausforderungen, die sich in der vulnerablen Phase der visuellen und anatomischen Entwicklung befindet. Die meisten bestehenden Untersuchungen sind entweder durch ihre retrospektive Natur oder durch kleine Fallzahlen den bekannten Unwägbarkeiten ausgesetzt. Neuere Untersuchungen wie die multizentrische randomisiert prospektive Infant-Aphakia-Treatment-Study (IATS) versuchen die Lücke zu schließen.

\subsubsection{Komplikationen}

Die postoperative Komplikationsrate ist ein wichtiger Faktor bei der Abwägung zur Implantation einer intraokulären Linse (IOL). Der Vergleich der vorhandenen Literatur wird, neben oben genannten Unwägbarkeiten, weiter erschwert. Dies geschieht unter anderem durch den Einbezug unterschiedlichster Kofaktoren, die unterschiedliche Begriffsdefinition, die verschiedenen Ein/Ausschlusskriterien und das breite Altersspektrum der Patienten. Untersucht wurden dabei insbesondere Unterschiede zwischen Patienten mit bilateraler und unilateraler Katarakt und Patienten mit primärer Implantation oder Kontaktlinsenversorgung mit möglicher sekundärer Linsenimplantation.

\subsubsection{Unilaterale Katarakt}

Lundvall und Kugelberg (Lundvall und Kugelberg 2002a) berichten in ihrer retrospektiven Arbeit über die langfristigen Auswirkungen (Follow-up: 4-9,5 Jahre) von im ersten Lebensjahr operierten Kindern mit unilateraler Katarakt unabhängig von der weiteren Versorgung. Dabei fallen durch sekundäre Linsenkapselveränderungen bedingte Re-Operationen bei neun von 30 Kindern auf. Außerdem werden neun Glaukom bedingte Folgeoperationen gezählt, insbesondere bei Patienten, die eine frühe Kataraktextraktion erfahren hatten und mit einem Schwerpunkt innerhalb der ersten sechs Monate nach Kataraktextraktion.

Lambert et al. (Lambert 2004) untersuchten 25 Patienten (primäre IOL zwölf vs. Kontaktlinse (CL) 13) mit Kataraktextraktion im Alter von mehr als sieben Monaten und einem Follow-up von 4,3 Jahren (3,3-5,5 Jahren). Die Untersuchung zeigte bei einer Gesamtzahl von 21 Patienten bezüglich der Komplikationsrate in der IOL-Gruppe (zehn Patienten) zwölf Eingriffe bei allen zehn Patienten, davon acht Membranektomien, zwei Glaukome und zwei IOL-Wechsel. In der CLGruppe (elf Patienten) gab es vier Eingriffe bei vier Patienten in Form von sekundären IOLImplantationen. Die Sehschärfe war in beiden Gruppen gleich. Verglichen die Autoren jedoch je 
zwei Patienten mit Kataraktextraktion innerhalb der ersten sechs Lebenswochen, zeigte sich in der IOL-Gruppe eine Entwicklung hin zu besserer Sehschärfe.

Auch Lundvall und Zetterström (Lundvall und Zetterström 2006) berichten in ihrer Untersuchung von 28 Kindern (24 mit unilateraler und vier mit bilateraler Katarakt) mit 31 Augen nach primärer IOL-Implantation von einem hohen Auftreten von operationsbedürftigen sekundären Linsenkapselveränderungen (67\% der Augen). Eine Linsenluxation ereignete sich bei zwei Patienten und ein Glaukom entwickelte sich in nur drei (9,7\%) Augen.

$\mathrm{Lu}$ et al. ( $\mathrm{Lu}$ et al. 2010) untersuchten retrospektiv das funktionelle Ergebnis und die Komplikationsrate bei Kindern mit primärer IOL-Implantation zwischen dem sechsten und zwölften Lebensmonat von 26 Augen (sechs unilaterale Katarakt) bei einer durchschnittlichen follow-up von 46,4 Monaten (22-79 Monate). Die Autoren fanden die Trübung der Sichtachse in sieben Augen (26,9\%) als häufigste Komplikation. Vier Augen benötigten eine erneute Pars-PlanaVitrektomie und ein Auge entwickelte ein Glaukom.

Die prospektiv randomisierte IATS mit 114 Patienten nach Kataraktextraktion unter sieben Monaten (57 primäre IOL vs. 57 CL) zeigte zum Ein-Jahres-Zeitpunkt fast ausschließlich ein hohes Aufkommen an Re-Operationen bedingt durch die Trübung der Sehachse (insges. 59 Eingriffe, 49 IOL vs. zehn CL) (Plager et al. 2011). Andersbedingte Re-Operationen traten in 13 Fällen (neun IOL vs. fünf CL) auf, davon waren fünf Glaukom bedingt. Insgesamt war die Re-Operationsrate in der IOL Gruppe zu diesem Zeitpunkt signifikant erhöht (36 Re-Operationen IOL (63\%) vs. sieben Re-Operationen CL (12\%); p<0,0001).

Zum Fünf-Jahres-Zeitpunkt der IATS (Plager et al. 2014) ergänzt sich die Zahl der gesamten Folgeoperationen um 18 Eingriffe (vier CL vs. 14 IOL) auf insgesamt 83 Eingriffe (17 CL vs. 66 IOL). Obwohl sich die Komplikationsrate der Zwischenzeit nicht signifikant unterschied (vier CL $(7 \%)$ vs. acht IOL (14\%); $\mathrm{p}=0,36)$, war der Unterschied zum Endzeitpunkt zugunsten der CLGruppe weiterhin signifikant (neun CL; 16\% vs. 41 IOL; 72\%; $p<0,0001$ ).

Bei genauerer Betrachtung kam es im Zeitraum zwischen dem Ein- und dem Fünf-JahresZeitpunkt zu elf Re-Operationen aufgrund von Trübungen in der Sehachse (acht IOL vs. drei CL) auf insgesamt 65 Eingriffe, drei Operationen zur Behandlung eines Glaukoms (zwei IOL vs. eine CL) auf insgesamt acht Eingriffe und zwei IOL Wechsel in der IOL Gruppe. Die häufigste Ursache zur Re-OP blieb daher die Trübung der Sehachse. Im Schwerpunkt lag die Rate der Re-OPs dabei im ersten postoperativen Jahr höher als im darauffolgenden Untersuchungszeitraum (Plager et al. 2014).

Im Vergleich zwischen den Gruppen konnten die Autoren zum Fünf-Jahres-Zeitpunkt keinen signifikanten Unterschied im Visus der Patienten feststellen (Lambert et al. 2014).

Rong et al. (Rong et al. 2015) untersuchten den Einfluss diverser Faktoren, unter anderem das Auftreten von Komplikationen bei Patienten mit sekundärer IOL-Implantation auf das Langzeitergebnis. Die retrospektive Untersuchung beinhaltet 76 Fälle von bilateraler Katarakt und 34 Fälle von unilateraler Katarakt, wobei in bilateralen Fällen je ein zufällig ausgewähltes Auge in 
die Untersuchung aufgenommen wurde. Im Zeitraum zwischen Kataraktextraktion und Implantation zeigten sich außer einer Trübung der Sehachse bei 13 Kindern (11,8\%) keine Komplikationen. Nach sekundärer IOL-Implantation kam es in sechs Fällen zur Trübung der Sichtachse (5,5\%) und in einem Fall zur IOL-Dislokation.

Die prospektiv populationsbasierte IoLunder2-Kohortenstudie (Solebo et al. 2015) mit einer unilateralen Kataraktgruppe von 90 Patienten (48 primäre IOL vs. 42 aphak), einem Median der Kataraktextraktion von neun Wochen (IQR 5 - 94) und einem Nachuntersuchungszeitraum von einem Jahr postoperativ berichtet die Trübung der Sehachse als häufigste Komplikation bei 34 Patienten (22 IOL vs. zwölf CL), gefolgt vom Glaukom bei sieben Patienten (drei IOL vs. vier CL). Die Implantation einer primären Intraokularlinse erhöhte auch hier die Wahrscheinlichkeit einer Re-Operation signifikant (adjusted OR 16,7; 95\%CI 4,1 - 68,9; $\mathrm{p}<0,001$ ). In Bezug auf die Häufigkeit des Auftretens des Glaukoms konnte nur ein signifikanter Mikrophthalmus (Achslänge $<16 \mathrm{~mm}$ ) als unabhängiger Prädiktor ermittelt werden (adjusted OR 12,1; 95\%CI 1,8 - 81,8; $\mathrm{p}=0,01)$. Die Autoren berichten bezüglich der unilateralen Katarakt keinen Unterschied beim Zielvisus im untersuchten Zeitraum.

\subsubsection{2 bilaterale Katarakt}

Lundvall und Kugelberg (Lundvall und Kugelberg 2002b) berichten über eine retrospektive Untersuchung, die das funktionelle Langzeitergebnis von 22 Kindern mit bilateraler Katarakt ermittelt, die vor dem zwölften Lebensmonat operiert wurden. Bei einem durchschnittlichen Follow-up von 6,5 Jahren (vier bis neun Jahre) entwickelten 21 Augen eine signifikante sekundäre Linsenkapselveränderung und sechs Augen ein operationsbedürftiges Glaukom. Die Autoren weisen bei frühem Operationszeitpunkt auf ein gutes visuelles Ergebnis hin, stellen jedoch außerdem ein erhöhtes Auftreten eines sekundären Glaukoms fest, wenn die Kataraktextraktion in der ersten Lebenswoche stattfand.

Lu et al. (Lu et al. 2010) untersuchten, wie schon oben genannt, retrospektiv das funktionelle Ergebnis und die Komplikationsrate in Kindern mit primärer IOL-Implantation zwischen dem sechsten und zwölften Lebensmonat. In der Studienpopulation fanden sich auch zehn Patienten mit bilateraler Katarakt (20 Augen). Eine Trübung der Sichtachse war auch hier die häufigste Komplikation.

Kim et al. (Kim et al. 2012) interessierten sich in einer retrospektiven Untersuchung für die Langzeitergebnisse von 37 Patienten (74 Augen) mit bilateraler Katarakt und Kontaktlinsenversorgung bzw. sekundärer IOL-Implantation. Die durchschnittliche Nachuntersuchungsdauer war 7,8 Jahre (vier bis zehn Jahre) und das mittlere Alter bei sekundärer IOL-Implantation 25 +/- 3,9 Monate. Mit 24 Augen (32\%) zeigte sich eine Glaukom-Entwicklung als häufigste postoperative Komplikation, jedoch benötigte nur ein Patient (zwei Augen) eine Glaukom-bedingte Re-Operation. Diese trat ebenso wie die Trübung der Sehachse bei einem Patienten in beiden Augen erst nach Implantation der sekundären IOL auf. 
Magli et al. (Magli et al. 2013) verglichen das Langzeitergebnis von primärer vs. sekundärer IOLImplantation bei Kindern mit bilateraler kongenitaler Katarakt. Bei sehr geringem Aufkommen von Komplikationen (sekundäre Linsenkapselveränderung und Glaukom je in nur einem Fall bei 30 Augen) konnten die Autoren keinen Unterschied bezüglich der Komplikationen in den beiden Gruppen feststellen.

In der IoLunder2-Studie (Solebo et al. 2015) fand sich bezüglich der Patienten mit bilateraler Katarakt (131 Patienten) die Trübung der optischen Achse mit 62 Augen bei 44 Kindern (41 Augen (37\%) IOL vs. 22 Augen (13\%) CL) ebenso wie in der unilateralen Gruppe als häufigste Komplikation, gefolgt vom Glaukom in 26 Augen von 21 Kindern (fünf Augen (5\% IOL) vs. 21 Augen (15\%) CL). Ähnlich der unilateralen Gruppe erhöht eine primäre IOL-Implantation die Wahrscheinlichkeit, eine Re-Operation wegen einer Trübung der Sehachse zu erfahren (adjusted OR 5,5; 95\%CI 2,2 - 13,2; p<0,01). Auch bezüglich der Patienten mit bilateraler Katarakt konnte kein Zusammenhang zwischen der Art der Versorgung und dem Visus zum Endzeitpunkt hergestellt werden.

In ihrer prospektiven Untersuchung von Patienten mit bilateraler kongenitaler Katarakt erforschten Zhang et al. (Zhang et al. 2016) den Einfluss diverser Faktoren, unter anderem der postoperativen Komplikationen, auf den finalen Visus. Die Untersuchung beinhaltete sowohl aphake Augen (164) als auch Augen mit primärer IOL-Implantation (zwölf). Neben einem frühen Operationszeitpunkt konnte eine geringe postoperative Komplikationsrate in einen Zusammenhang mit besseren Visus-Ergebnissen gestellt werden.

\subsubsection{Bulbuslänge}

Die Achslänge ist ein signifikanter Indikator der Augenentwicklung. Ein besseres Verständnis der Achslänge und ihrer Entwicklung ermöglichen eine Optimierung der Behandlungsstrategien.

Wie schon im Kapitel Komplikationen erwähnt, erschweren die unterschiedlichen Studiendesigns den Vergleich der Literatur. Der Nachweis, dass Achslängenmessungen im ambulanten Rahmen denen unter Vollnarkose durchgeführten entsprechen (Kinori et al. 2015), erleichtern die Analyse.

Griener et al. (Griener et al. 1999) untersuchten in einer kleinen Population die Auswirkungen einer primären IOL-Implantation auf das Achswachstum bei elf Kindern mit unilateraler Katarakt im Vergleich zur gesunden Gegenseite. Die Patienten wurden im Alter von zwei bis vier Monaten operiert und im Mittel nach fünf bis sechs Jahren nachuntersucht. Dabei zeigte sich ein vermindertes Wachstum in den IOL-Augen. Die Autoren schlossen daraus, dass bei IOLImplantation Aufgrund des verminderten Achswachstums mit einem geringeren Myopic Shift gerechnet werden müsse.

Vasavada et al. (Vasavada et al. 2004) untersuchten in einer prospektiven Fallserie von 79 Kindern mit 158 Augen den Einfluss von Kataraktchirurgie bei Kindern mit kongenitalem Katarakt auf die Entwicklung der Achslänge. Die Autoren führten den Begriff der RAG (rate of axial growth) ein als prozentuale Entsprechung der Differenz zwischen der mittleren präoperativen AL (Achslänge) 
und der mittleren AL zum Zeitpunkt des letzten Follow-up dividiert durch die durchschnittliche präoperative Achslänge. Diese wurden auf Korrelationen zu folgenden Faktoren hin untersucht: Alter des Kindes zum Zeitpunkt der Operation, betroffene Seite und Einfluss einer Trübung der Sichtachse. Im Ergebnis zeigte sich, dass Kinder mit einem Operationszeitpunkt im ersten Lebensjahr eine höhere RAG aufwiesen, die bis zum zweiten postoperativen Jahr anstieg. Im Vergleich der unilateralen IOL-Augen mit ihrer gesunden Gegenseite zeigten Vasavada et al. ein größeres Wachstum der IOL-Augen. Die Trübung der Sehachse zeigte sich ohne Einfluss auf die RAG.

Trivedi und Wilson (Trivedi und Wilson 2007b) ermittelten in ihrer Untersuchung von 74 Augen, dass Augen mit kurzer präoperativer AL eine höheres postoperatives Wachstum und Augen mit langer präoperativer AL eine geringeres postoperatives Wachstum aufweisen, so dass sich im Vergleich zur gesunden Gegenseite insgesamt eine Tendenz der Differenz zu Null hin ergibt.

Capozzi et al. (Capozzi et al. 2008) untersuchten retrospektiv die Achslänge von Kindern mit kongenitaler Katarakt in den ersten 42 Lebensmonaten mit einer Gesamtaugenzahl von 180 Augen. Die Autoren fanden erwartungsgemäß einen signifikanten Unterschied der Achslänge in unterschiedlichem Lebensalter. Außerdem zeigte sich eine signifikant größere Achsenlänge in unilateralen als in bilateralen Fällen $(\mathrm{p}=0,029)$.

Sminia et al. (Sminia et al. 2010) befassten sich mit 90 Augen von 45 Kindern mit unilateraler Katarakt und unterteilten dabei zwei Gruppen nach dem Operationszeitpunkt. Zeigte sich der Gruppe mit einer OP jünger als 18 Monate (durchschnittlicher follow-up 4,3 +/- 3,2 Jahre) im absoluten Wachstum noch ein grenzwertig signifikanter Unterschied zwischen operiertem und nicht operiertem Auge $(\mathrm{p}=0,049)$, konnte dieser beim Betrachten der RAG nicht mehr nachgewiesen werden $(p=0,25)$. In der Gruppe mit einer OP nach 18 Monaten und älter zeigten sich insgesamt keine Unterschiede.

Kim et al. (Kim et al. 2012) untersuchte in einer Multivariatanalyse den Zusammenhang von verschiedenen Faktoren zum finalen Visus und konnte dabei einen solchen bezüglich der Achslänge feststellen. Eine längere Achse korrelierte mit einem schlechteren Visus.

Auch im Zuge der prospektiv randomisierten IATS wurde das Achswachstum der 114 Patienten mit unilateraler Katarakt untersucht. In der Baseline-Untersuchung zeigte sich noch kein Unterschied zwischen den Gruppen IOL und CL. Augen mit Katarakt waren hier durchschnittlich 0,6 mm kürzer als ihre gesunden Entsprechungen (95\%CI 0,4 - 0,6 mm; p<0,0001). Zum EinJahres-Zeitpunkt zeigten sich die Augen der CL-Gruppe im Durchschnitt 0,6 mm kürzer als die der IOL-Gruppe $(\mathrm{p}=0,009)$ (Lambert et al. 2012).

Zum Fünf-Jahres-Zeitpunkt gab es keinen Unterschied in der Wachstumsdifferenz (Baseline zum letzten Follow-up) zwischen behandeltem und unbehandeltem Auge. Die Längenänderung der beiden Gruppen (3,4 mm IOL vs. 3,2 mm CL; p=0,53) war ähnlich und korrelierte nicht mit dem finalen Visus. Dagegen zeigten sowohl Augen mit zusätzlichen Eingriffen zur Klärung einer 
Trübung der Sehachse als auch Augen mit Glaukom ein signifikant größeres Wachstum (Wilson et al. 2017).

Lin et al. (Lin et al. 2016) untersuchten prospektiv eine Population von 1586 Patienten mit 3172 Augen jedoch mit einer oberen Altersgrenze von 18 Jahren. Aus dieser großen Population leiteten die Autoren eine logarithmische Korrelation zwischen der Achslänge (AL) und dem Patientenalter ab. Sie fanden um das zweite Lebensjahr einen Wendepunkt in der Wachstumsrate. Außerdem berichteten sie eine längere AL des betroffenen Auges in unilateralen Fällen bezüglich der Gegenseite und bezüglich der bilateralen Augen sowie eine längere AL bei Jungen als bei Mädchen von Kindern zwischen dem zweiten bis sechsten Lebensjahr.

\subsubsection{Sphärisches Äquivalent / Myopic Shift}

Mit Hilfe des sphärischen Äquivalents als Maß des Refraktionsfehlers kann man die Gesamtbrechkraft des Auges in einer Zahl darstellen. Es lassen sich hieraus Aussagen über den Erfolg der Berechnung der benötigten Kraft der Intraokularlinse sowie über die Entwicklung des Verbundes Auge als Brechmittel ableiten.

McClatchey et al. (McClatchey et al. 2000) verglichen in ihrer Untersuchung die rate of refractive growth (RRG) von aphaken und pseudophaken Augen. Sie ermittelten eine leicht signifikante geringere RRG in pseudophaken Augen (-4,6 dpt vs. 5,7 dpt; $\mathrm{p}=0,03)$. Die Signifikanz dieses Trends hob sich jedoch auf, als die Gruppen mit Kataraktextraktion jünger als sechs Lebensmonate und älter als sechs Lebensmonate getrennt untersucht wurden.

Auch Superstein et al. (Superstein et al. 2002) fanden in einer großen retrospektiven Untersuchung einen geringeren Myopic Shift in pseudophaken Augen, mussten jedoch ihre Aussagen aus Mangel an Daten auf Kinder mit Pseudophakie älter als zwei Jahre begrenzen.

Weakley et al. (Weakley et al. 2003) untersuchten den Zusammenhang zwischen der RRG und dem Zielvisus. Sie schlossen, dass die RRG signifikant mit dem Zielvisus korreliert. Dabei zeigten Augen mit schlechterem Visus eine größere RRG.

Magli et al. (Magli et al. 2013) verglichen Patienten mit primärer und sekundärer IOL und stellten fest, dass sich bei primär implantierten Kindern ein signifikant größerer Myopic Shift aufzeigt als bei sekundärer Implantation $(26 \%$ vs. $11,1 \%$, p <0,001) und dies bei einem Refraktionsfehler beim letzten Kontakt von $-2,5+/-3$ dpt primär vs. $0,4+/-2,3$ dpt sekundär bei $\mathrm{p}=0,02$.

Kim et al. (Kim et al. 2012) stellten zum Zeitpunkt drei Monate nach IOL-Implantation bei bilateraler kongenitaler Katarakt eine Abweichung vom kalkulierten Zielvisus um mehr als 2 dpt in den meisten der untersuchten Augen fest (52 Augen, 71\%).

$\mathrm{Zu}$ ähnlichen Ergebnissen kamen die Autoren um Rong et al. (Rong et al. 2015) in ihrer Untersuchung von sekundär implantierten IOL. Auch sie konnten eine Assoziation zwischen dem Visus und dem Refraktionsfehler zum letzten Untersuchungszeitpunkt darstellen. In bilateralen Fällen kam es zu Refraktionsfehlern von 3,11 dpt + / - 2,45 dpt und in unilateralen Fällen zu Fehlern von $-2,39 \mathrm{dpt}+/-2,77 \mathrm{dpt}$. 
Im Rahmen der IATS tragen zwei Untersuchungen zum Themenbereich bei. Lambert et al. (Lambert et al. 2016) untersuchten die RRG und kamen zu dem Schluss, dass es zu keinem Unterschied zwischen den Gruppen kam. Sie konnten zeigen, dass in aphaken Augen, jedoch nicht in pseudophaken Augen, ein schlechtes Visusergebnis mit einer hohen RRG ähnlich den Untersuchungen von Weakley et al. assoziiert war.

Weakley et al. (Weakley et al. 2017) stellten in der Untersuchung des Myopic Shift fest, dass die Rate des Myopic Shift in den ersten eineinhalb Lebensjahren am höchsten ist. Anhand der gewonnenen Daten schlagen sie veränderte postoperative hypermetropische Zielwerte vor, jedoch unter der hohen Wahrscheinlichkeit, dass die betroffenen Kinder aufgrund der hohen Variabilität trotz dessen eine refraktäre Korrektur benötigen.

\subsection{Hintergrund und Fragestellung}

Hintergrund dieser Arbeit ist die Tatsache, dass es keinen evidenzbasierten Konsens darüber gibt, ob Kinder mit kongenitaler Katarakt bereits in den ersten beiden Lebensjahren eine Intraokularlinse erhalten sollen oder ob sie zunächst mit Kontaktlinse versorgt werden können.

Die folgende Untersuchung am Patientenkollektiv der Universität Göttingen soll dazu beitragen, Faktoren zu identifizieren, um die intra- und postoperative Versorgung von Kindern mit operationswürdiger kongenitaler Katarakt zu verbessern. 


\section{Material und Methoden}

In der vorliegenden Arbeit werden Patienten mit der Diagnose ,kongenitale Katarakt' untersucht, die im ersten Lebensjahr aufgrund der Linsentrübung operiert wurden. Eingeschlossen wurden Patienten, die sich von 1990 bis 2015 in Behandlung der Augenklinik der Universitätsmedizin Göttingen befanden. Dabei wurde im Jahr 1985 mit klinikinternen Aufzeichnungen dieser Patienten begonnen. Die Aktendurchsicht erfolgte anhand dieser klinikinternen Aufzeichnungen.

Erfasst wurden dabei folgende Parameter:

1. Name des Patienten

2. Patientennummer

3. Geschlecht des Patienten

4. Geburtsdatum des Patienten

5. Seite des betroffenen Auges

6. Zeitpunkt der Operation aus dem Operationsprotokoll

7. Durchgeführter Eingriff aus dem Operationsprotokoll

8. Eingriffsursache aus dem Operationsprotokoll

9. Datum der letzten Verlaufsuntersuchung im Haus

10. Bulbuslänge

a. in $\mathrm{mm}$

b. Zeitpunkt der Messung

11. Refraktion

a. als sphärisches Äquivalent

(Der Begriff sphärisches Äquivalent bezeichnet die durchschnittliche sphärische Power einer sphärosylindrischen Linse, d. b. [Wert der Aberrationen 1. Ordnung wie Myopie, Hyperopie]

+ [Wert der Aberrationen 2. Ordnung (Astigmatismus) / 2]; Beispiel: [- 2 dpt Myopie] + [0,5 dpt Astigmatismus / 2] = -2,25 dpt))

b. Zeitpunkt der Messung

Um eine möglichst einheitliche und vergleichbare Situation zu erreichen, wurden folgende Einflüsse auf den Datensatz vorgenommen.

Erfasst wurden:

- nur Operationen und Messungen, die in der Augenklinik der Universitätsmedizin Göttingen durchgeführt wurden

Ausgeschlossen wurden alle:

- Patienten, deren Lentektomie bzw. primäre Linsenimplantation nicht in Göttingen durchgeführt wurde

- Patienten, bei denen die Diagnose kongenitale Katarakt nicht klar benannt war

- Patienten, bei denen zwischen dem Zeitpunkt der Geburt und der Lentektomie bzw. der primären Linsenimplantation mehr als ein Jahr vergangen war 
- Patienten, die ihre Lentektomie bzw. primäre Linsenimplantation vor 1990 erhielten (aufgrund Speicherfrist des Archives der Universitätsmedizin Göttingen von 25 Jahren sowie veränderten Dokumentationsgegebenheiten und Aktenführung)

Folgende Parameter wurden aus den Grunddaten gebildet, gegliedert und gruppiert und dienten dann der weiteren Untersuchung:

1. Patienten ID (ungerichtet durchlaufenden Nummer der Patienten)

2. Augen ID (ungerichtet durchlaufende Nummer der Augen)

3. Seite des betroffenen Auges

4. Geschlecht

5. Geburtsdatum

6. Datum der Kataraktextraktion

7. Datum der Linsenimplantation

8. Art der Linsenversorgung

1. primär (Zeitpunkt von Kataraktextraktion und Implantation gleich)

2. sekundär (Zeitpunkt von Kataraktextraktion und Implantation unterschiedlich)

3. aphak (Patienten ohne Implantation)

9. Komplikation
a. Nachstar (als Entsprechung einer sekundären Linsenkapselveränderung)
b. Sekundärglaukom
c. IOL- Luxation/Subluxation
d. Synechien
e. Netzhautablösung
f. Glaskörper-Blutung
g. Glaskörper-Prolaps
h. Hornhaut-Eintrübung
i. andere

10. Operationen
a. Nachstarentfernung
b. Zyklokryokoagulation
c. Zyklophotokoagulation (CPC)
d. HKL-Reposition
e. HKL-Einnähung / Austausch
f. Keratoplastik
g. filtrierende Glaukom-OP
h. Vitrektomie
i. Synechiolyse
j. andere

11. Bulbuslänge

a. in $\mathrm{mm}$

b. gruppiert nach Lebensalter: null bis einschließlich zweites Lebensjahr, zweites bis einschließlich sechstes Lebensjahr, sieben Jahre und älter

c. Rate of axial growth

i. Einschlusskriterien: Baseline-Untersuchung bei +/- eine Woche um Kataraktextraktion, mindestens zwei Jahre nachuntersucht

ii. Achswachtum $=$ Follow-up - Baseline

iii. Achswachstumsrate $=($ Achswachstum $/$ Baseline $) \times 100$

12. Sphärisches Äquivalent (Als Entsprechung des gemessenen Brechungsfehlers in dpt, s. o.)

a. Zeitpunkt der Bestimmung

b. gruppiert nach Lebensalter: null bis einschließlich zweites Lebensjahr, drittes bis einschließlich sechstes Lebensjahr, sieben Jahre und älter 
Die Auswertung erfolgte nach zwei Primärkriterien:

1. Art der operativen Versorgung (primär/sekundär/keine)

2. mögliche Komplikationen, die zur Re-Operation führten

Kam es im Zeitraum der Untersuchung zu Komplikationen, waren folgende Nebenkriterien von Interesse:

1. Art der Komplikation

2. Art der Re-Operation

3. Zeitpunkt der Re-Operation

Der Datensatz ermöglichte des Weiteren die Untersuchung zweier Sekundärkriterien:

1. Verlauf des Bulbuswachstums (zur Untersuchung von Wachstumsgeschwindigkeit und Bulbusgröße der Gruppen)

2. sphärisches Äquivalent zum letztmöglichen Zeitpunkt nach Lentektomie gemessen (zur Bewertung der möglichen Unterschiede zwischen den Gruppen)

Die Patienten und Augen wurden einzeln mit einer fortlaufenden Nummer markiert und Namen und Patientennummer gelöscht, um im Rahmen der weiteren statistischen Untersuchung bestmögliche Anonymität herzustellen.

Die statistische Auswertung erfolgte mittels absoluter und relativer Häufigkeit, Mittelwert uns Standardabweichung, t-Test und Varianzanalyse.

Die statistische Auswertung erfolgte mit Hilfe des Instituts für anwendungsorientierte Forschung und klinische Studien GmbH (IFS), ein ehemaliges Tochterunternehmen der Georg-AugustUniversität Göttingen, Stiftung Öffentlichen Rechts. 


\section{Ergebnisse}

Die Untersuchung basiert auf einer klinikintern geführten Liste von Patienten mit der Diagnose kongenitale Katarakt, die zwischen 1985 und 2012 an der Augenklinik der Universitätsmedizin Göttingen (UMG) operiert wurden. Die Liste umfasst 166 Patienten. Die durchschnittliche Nachbeobachtungszeit betrug 8,77 Jahre.

\subsection{Patienten}

Nach Anwendung der Ein- und Ausschlusskriterien (siehe Kapitel 2) verblieben 54 Patienten, welche bezüglich der Geschlechter gleichmäßig verteilt waren (55,6\% vs. 44,4\%). Die vier primär versorgten Patienten waren alle männlich (Tabelle 1).

Tabelle 1 Verteilung nach Geschlecht und Versorgungtyp

\begin{tabular}{|l|l|l|l|}
\hline \multicolumn{4}{|c|}{ Geschlecht } \\
IOL & \multicolumn{1}{c|}{ m } & \multicolumn{1}{c|}{ w } & \multicolumn{1}{c|}{ Total } \\
\hline aphak & $10(66,6 \%)$ & $5(33,3 \%)$ & $15(27,8 \%)$ \\
\hline primär & $4(100 \%)$ & 0 & $4(7,4 \%)$ \\
\hline sekundär & $16(45,7 \%)$ & $19(54,3 \%)$ & $35(64,8 \%)$ \\
\hline Total & $30(55,6 \%)$ & $24(44,4 \%)$ & $54(100 \%)$ \\
\hline
\end{tabular}

20 Patienten hatten eine bilaterale und 34 eine unilaterale kongenitale Katarakt (37\% vs. 63\%). Die Mehrheit der Patienten sowohl der bi- als auch der unilateralen Gruppe erhielten sekundär eine IOL (65\% bilateral vs. 64,7\% unilateral). Nur wenige Patienten (vier) erhielten eine primäre IOLImplantation, drei davon hatten eine unilaterale Katarakt. Der Anteil der 15 aphak belassenen Patienten ist in beiden Gruppen annähernd gleich (30\% vs. 26.5\%) (Tabelle 2).

Tabelle 2 Verteilung der Patienten nach Lateralität und Versorgungstyp

\begin{tabular}{|c|c|c|c|}
\hline \multirow[b]{2}{*}{ IOL } & \multicolumn{2}{|c|}{ Katarakt } & \multirow[b]{2}{*}{ Total } \\
\hline & bilateral & unilateral & \\
\hline aphak & $6(40 \%)$ & $9(60 \%)$ & $15(27,8 \%)$ \\
\hline primär & $1(25 \%)$ & $3(75 \%)$ & $4(7,4 \%)$ \\
\hline sekundär & $13(37,1 \%)$ & $22(62,9 \%)$ & $35(64,8 \%)$ \\
\hline Total & 20 (37\%) & 34 (63\%) & $54(100 \%)$ \\
\hline
\end{tabular}

Die durchschnittliche Dauer der Nachuntersuchung nach Kataraktextraktion zeigt Tabelle 3 bezüglich der Art der Versorgung. In Tabelle 4 ist die Nachbeobachtungszeit dargestellt, getrennt nach einseitiger oder beidseitiger Katarakt. Dabei sticht der vergleichsweise kurze Nachuntersuchungszeitraum der primär versorgten Patienten hervor. 
Tabelle 3 Durchschnittliche Nachuntersuchungsdauer in Jahren nach Versorgungstyp

\begin{tabular}{|lr|r|r|}
\hline \multicolumn{1}{|c|}{ IOL } & N & Mean \pm SD & $95 \%$ Cl \\
\hline aphak & 15 & $6,44 \pm 4,94$ & $3,71-9,18$ \\
\hline primär & 4 & $3,76 \pm 3,84$ & $-2,35-9,87$ \\
\hline sekundär & 35 & $8,39 \pm 4,36$ & $6,89-9,89$ \\
\hline Total & 54 & $7,50 \pm 4,62$ & $6,24-8,77$ \\
\hline
\end{tabular}

Tabelle 4 Durchschnittliche Nachuntersuchungsdauer in Jahren nach Lateralität

\begin{tabular}{|l|c|c|c|}
\hline $\begin{array}{c}\text { uni- } \\
\text { /bilateral }\end{array}$ & N & Mean \pm SD & 95\% Cl \\
\hline bilateral & 20 & $7,97 \pm 4,43$ & $5,89-10,04$ \\
\hline unilateral & 34 & $7,23 \pm 4,78$ & $5,56-8,90$ \\
\hline Total & 54 & $7,50 \pm 4,62$ & $6,24-8,77$ \\
\hline
\end{tabular}

Entsprechend zu den Ergebnissen in Tabelle 3 ist auch das Alter bei der Abschlussuntersuchung erwartungsgemäß geringer bei den 4 Jungen mit primärer IOL-Implantation (aphak 6,83 +/- 4,92 Lj. vs. primär 4,31 +/- 3,75 Lj. vs. sekundär 8,74 +/- 4,34 Lj.; bilateral 8,31 +/- 4,47 Lj. vs. unilateral 7,64 +/- 4,71 Lj.)

\subsection{Augen}

Die 54 Patienten repräsentieren 74 Augen, die im Folgenden einzeln untersucht werden. 40 Augen $(54,1 \%)$ stammen aus der Gruppe der bilateralen kongenitalen Katarakt, 34 Augen (45,9\%) aus der Gruppe der unilateralen kongenitalen Katarakt. Verglichen mit der Verteilung der Patienten zwischen uni- und bilateraler Katarakt (siehe Tabelle 2) nähern sich die Zahlen bezogen auf die Augen einer gleichmäßigen Verteilung weiter an (Tabelle 5).

Tabelle 5 Verteilung der Augen nach Lateralität und Versorgungstyp

\begin{tabular}{|l|l|l|l|}
\hline \multicolumn{1}{|c|}{ Katarakt } & \multicolumn{1}{c|}{} \\
\hline \multicolumn{1}{|c|}{ IOL } & \multicolumn{1}{|c|}{ bilateral } & \multicolumn{1}{c|}{ unilateral } & \multicolumn{1}{c|}{ Total } \\
\hline aphak & $12(57,1 \%)$ & $9(42,9 \%)$ & $21(28,4 \%)$ \\
\hline primär & $2(40 \%)$ & $3(60 \%)$ & $5(6,7 \%)$ \\
\hline sekundär & $26(54,2 \%)$ & $22(45,8 \%)$ & $48(64,9 \%)$ \\
\hline Total & $40(54,1 \%)$ & $34(45,9 \%)$ & $74(100 \%)$ \\
\hline
\end{tabular}

Das Alter zum Zeitpunkt der Kataraktextraktion war in unserer Population im Mittel bei 134,73 Tagen, also rund 19 Wochen (Tabelle 6). Es zeigt sich, dass die Kataraktextraktion und 
Implantation einer primären Linse zu einem eher späteren Zeitpunkt erfolgte als die Kataraktextraktion in den anderen Fällen (ca. 27 Wochen vs. ca. 18 - 19 Wochen). Auch die Patienten mit bilateraler Katarakt wurden im Durchschnitt früher behandelt (ca. 17 Wochen vs. ca. 21 Wochen) (Tabelle 7).

Tabelle 6 Alter bei Kataraktextraktion in Tagen nach Versorgungstyp

\begin{tabular}{|l|r|c|c|}
\hline \multicolumn{1}{|c|}{ IOL } & N & Mean \pm SD & $95 \%$ Cl \\
\hline aphak & 21 & $135,29 \pm 90,09$ & $94,28-176,30$ \\
\hline primär & 5 & $192,00 \pm 47,46$ & $133,07-250,93$ \\
\hline sekundär & 48 & $128,52 \pm 88,05$ & $102,95-154,09$ \\
\hline Total & 74 & $134,73 \pm 87,12$ & $114,55-154,91$ \\
\hline
\end{tabular}

Tabelle 7 Alter bei Kataraktextraktion in Tagen nach Lateralität

\begin{tabular}{|l|c|c|c|}
\hline $\begin{array}{c}\text { Uni- } \\
\text { /bilateral }\end{array}$ & $\mathbf{N}$ & Mean \pm SD & $95 \% \mathbf{C l}$ \\
\hline bilateral & 40 & $123,18 \pm 82,57$ & $96,77-149,58$ \\
\hline unilateral & 34 & $148,32 \pm 91,54$ & $116,39-180,26$ \\
\hline Total & 74 & $134,73 \pm 87,12$ & $114,55-154,91$ \\
\hline
\end{tabular}

Sehr ähnlich der Verteilung nach Patienten (Tabelle 3 und 4) zeigt die Aufstellung der Augen bezüglich des Nachuntersuchungszeitraums einen kürzeren Nachuntersuchungszeitraum der primär implantierten Kinder (aphak 6,06 +/- 4,67 Lj. vs. primär 4,82 +/- 4,08 Lj. vs. sekundär 8,61 +/- 4,31 Lj.; bilateral 7,97 +/- 4,38 Lj. vs. unilateral 7,23 +/- 4,78 Lj.).

Die Dauer von der Kataraktextraktion bis zur Implantation der Linse in der Gruppe der sekundärimplantierten IOL war im Mittel 4,36 +/- 2,33 Jahre (95\% CI 3,68 - 5,03 Jahre).

Vergleicht man nun den Zeitraum vom Zeitpunkt der Linsenimplantation bis zur letztmöglichen Untersuchung der primär und sekundär implantierten Kinder, zeigt sich im Unterschied zum Vergleich des gesamten Nachuntersuchungszeitraums dieser Kinder (siehe oben), aber kaum mehr ein Unterschied zwischen den Gruppen. Nach IOL-Implantation weisen alle Patienten einen ähnlich langen Nachuntersuchungszeitraum auf (primär 4,82 +/- 4,08 Lj. vs. sekundär 4,25 +/4,52 Lj.; bilateral 4,34 +/- 4,51 Lj. vs. unilateral 4,17 +/- 4,48 Lj.).

\subsection{Komplikationen}

Abbildung 13 zeigt den Anteil der Augen, die mindestens eine Komplikation und demnach eine Re-Operation hatten. In Abbildung 14 wird der prozentuale Anteil der Komplikationen dargestellt, der in der jeweiligen Operationsgruppe aufgetreten ist. Sowohl der Chi-Quadrat-Test $(p=0,0409)$ 
als auch der Fischer-Exakt-Test $(\mathrm{p}=0,0423)$ bestätigen bei einem Signifikanzniveau von 5\%, einen Unterschied im Komplikationsauftreten zwischen den drei Arten der Versorgung.

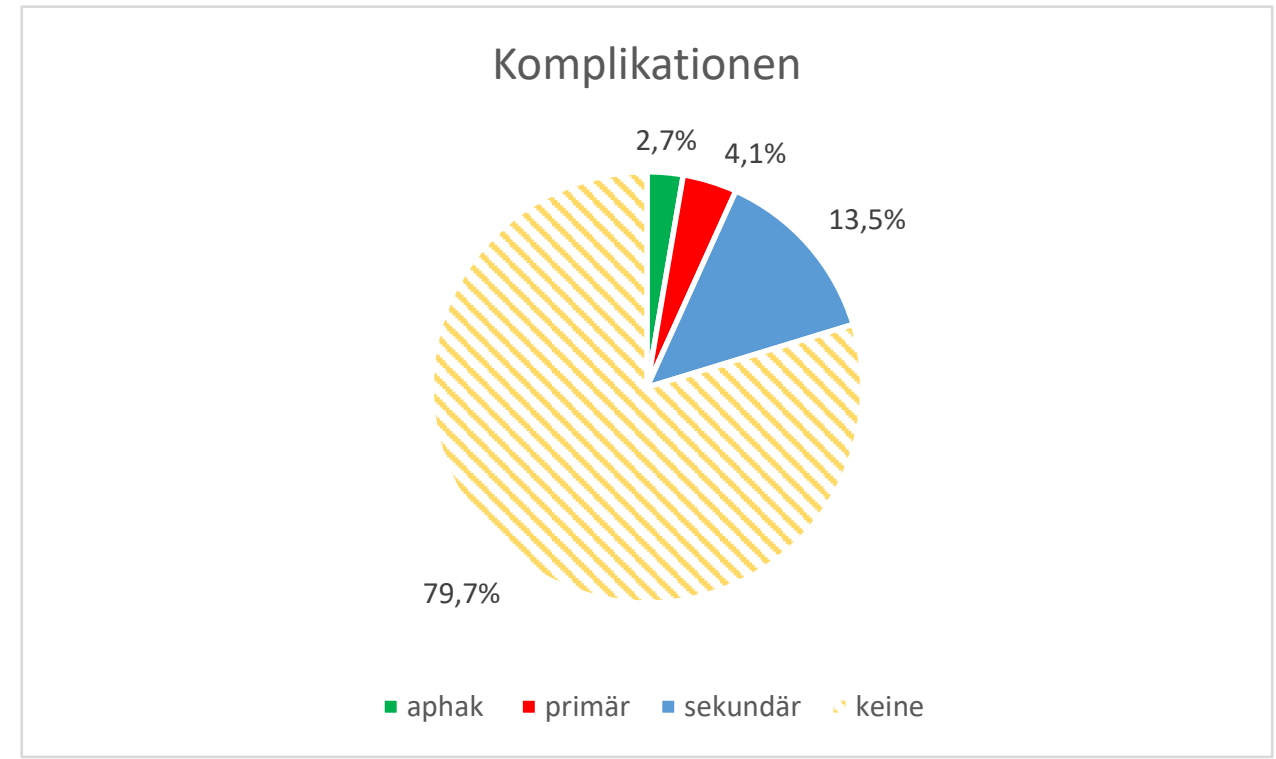

Abbildung 13 Anteil der Komplikationen an der Gesamtaugenzahl

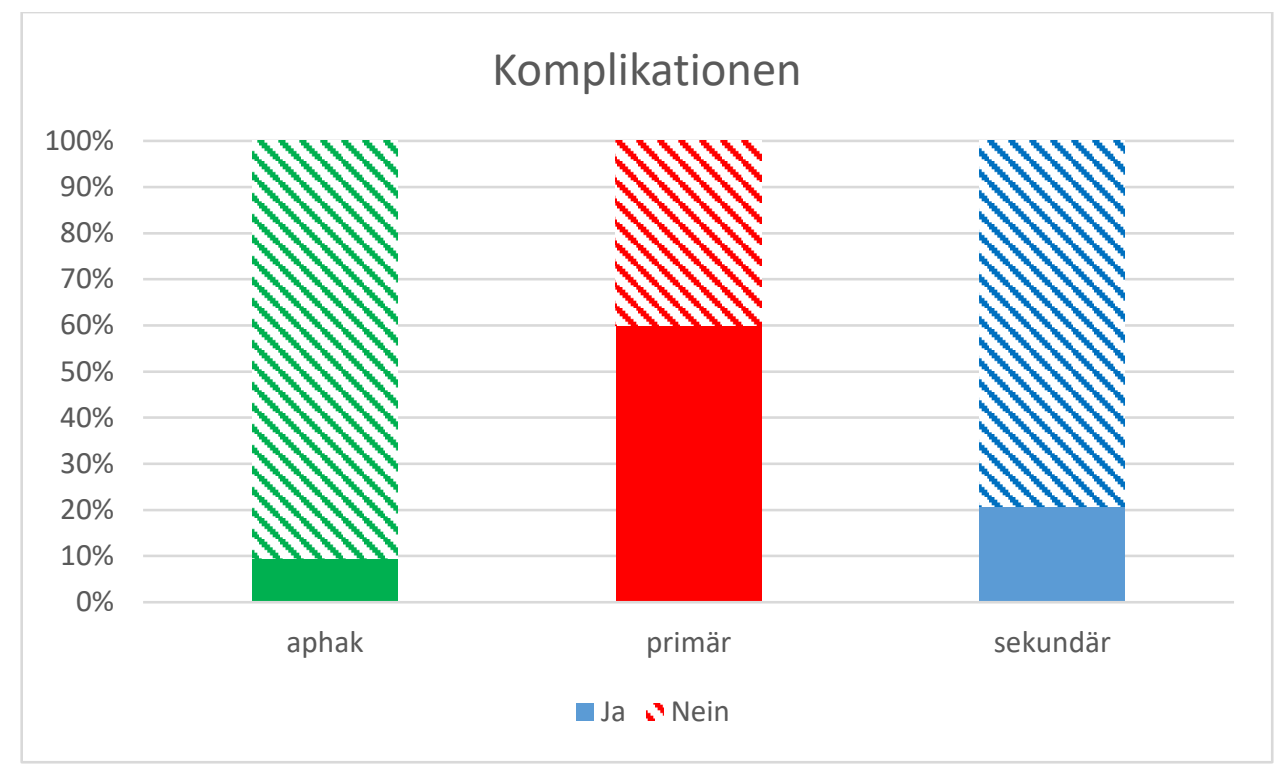

Abbildung 14 Anteil der Komplikationen nach Versorgungstyp

Im paarweisen Vergleich der Gruppen zeigt sich zwischen den aphaken Patienten und den primär implantierten Kindern ein signifikanter Unterschied (Fischer-Exakt-Test: p=0,0335). Vergleicht man die primär mit den sekundär implantierten Augen, ist der Chi-Quadrat-Test gerade eben nicht signifikant $(p=0,0527)$, der Fisher-Exakt-Test jedoch nicht signifikant $(p=0,0882)$, was auf eine unzureichend große Testmenge hinweisen könnte. Im Vergleich von aphaken Patienten und sekundär Versorgten kann kein Unterschied zwischen den Gruppen nachgewiesen werden. 
Bei der Untersuchung des Auftretens von mindestens einer Komplikation bezüglich der Patienten in den drei Gruppen kann kein signifikanter Unterschied festgestellt werden (Fischer-Exakt-Test $\mathrm{p}=0,2689)$.

Abbildung 15 zeigt den Anteil der Augen mit Komplikationen, je nach Art der Versorgung, der jeweiligen Untergruppe. Auffallend ist der hohe Anteil an sekundären Linsenkapselveränderungen und Synechien in der primären IOL-Gruppe. Es kann jedoch nur bezüglich der Komplikation der sekundären Linsenkapselveränderung ein signifikanter Unterschied zwischen den Gruppen festgestellt werden $(p=0,007)$. Dieser Unterschied entsteht bei genauerer Betrachtung in den jeweiligen Vergleichen mit primär versorgten Augen (aphak vs. primär: Fisher-Exakt-Test $\mathrm{p}=0,0335$; sekundär vs. primär: Fisher-Exakt-Test $\mathrm{p}=0,0078$; aphak vs. sekundär: Fisher-ExaktTest: $\mathrm{p}=0,6364)$.

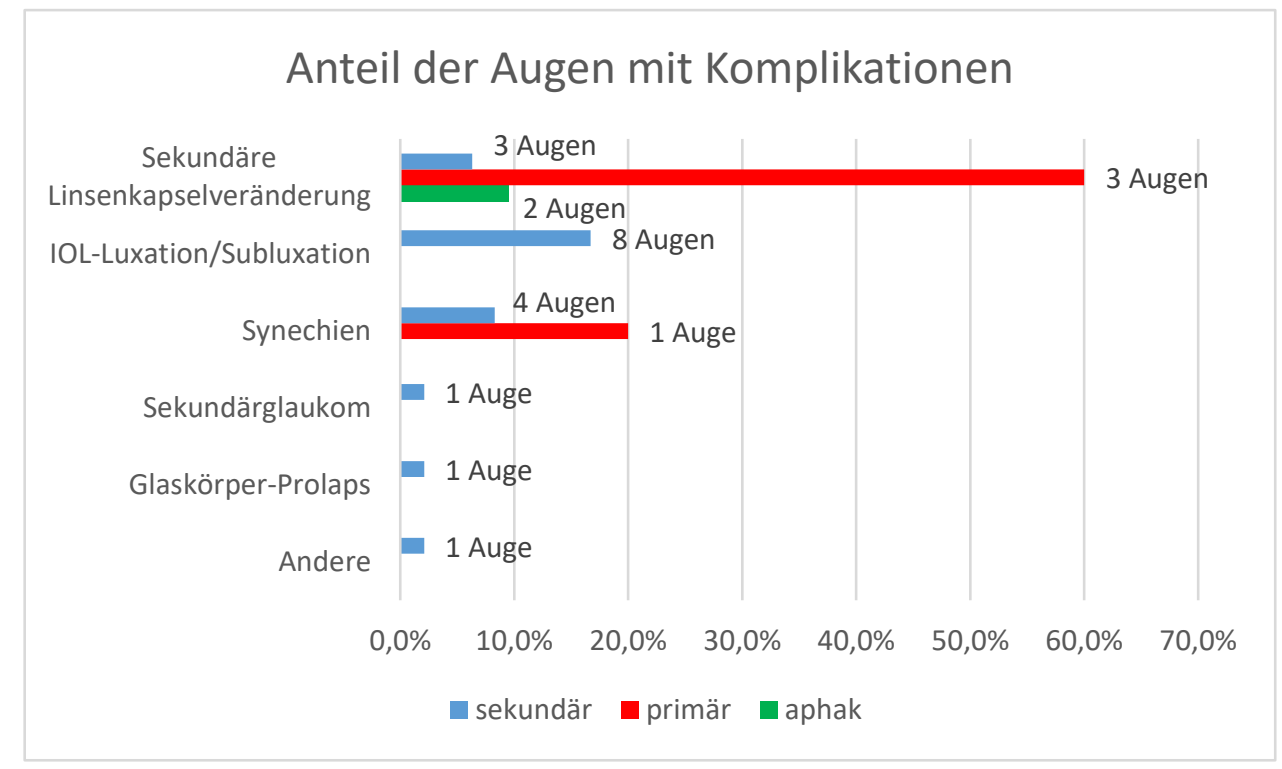

Abbildung 15 Auftreten der Komplikationen nach Versorgungstyp

Abbildung 16 ermöglicht einen Vergleich der Komplikationen bezüglich der Patienten mit bilateraler und unilateraler Katarakt. Patienten mit bilateraler Katarakt scheinen dabei häufiger von Komplikationen betroffen zu sein. Dies Bestätigt sich bei der Untersuchung von Patienten mit mindestens einer Komplikation mittels Fisher-Exakt-Test bei einem Signifikanzniveau von 5\% $(\mathrm{p}=0,0386)$. 


\section{Anteil der Patienten mit Komplikationen}

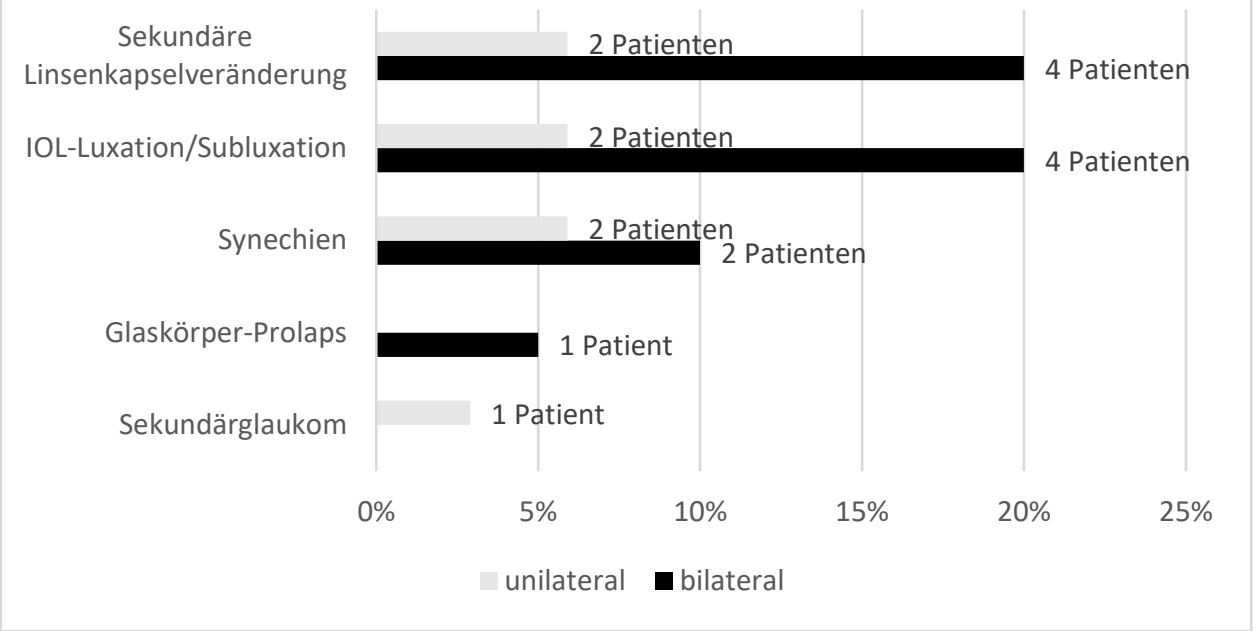

Abbildung 16 Auftreten der Komplikationen nach Lateralität

Insgesamt kam es in 33 Fällen zu operationsbedingten Komplikationen, die einen erneuten Eingriff erforderlich machten. Dabei waren bei 19 Patienten 24 Augen betroffen. Abbildung 17 zeigt die Verteilung der Komplikationen.

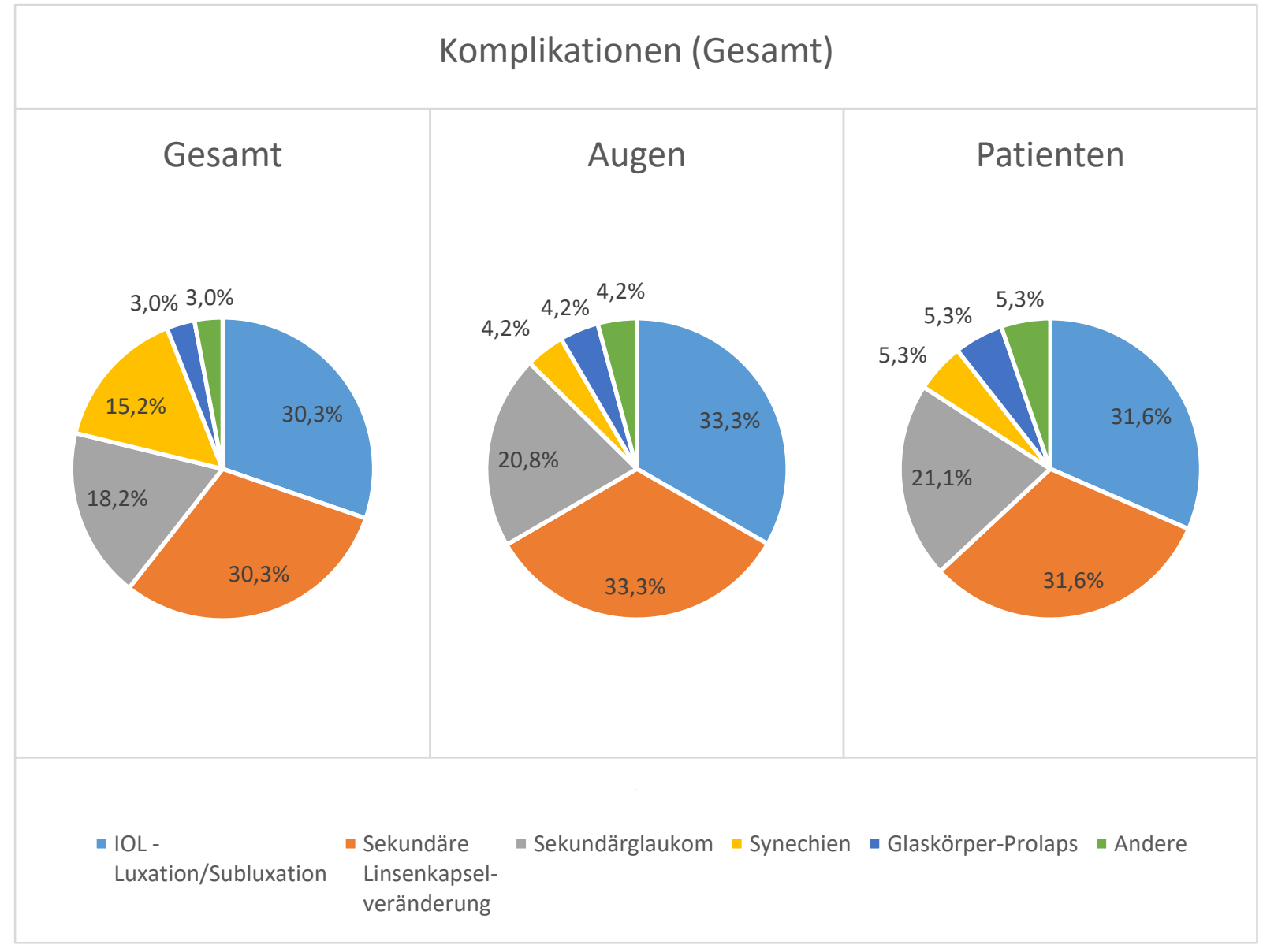

Abbildung 17 Prozentuale Verteilung bezüglich aller Komplikationen, der komplikationsbehafteten Augen und der von Komplikationen betroffenen Patienten 
Die Unterschiede zwischen den Anteilen an den Gesamtkomplikationen, den Anteilen bezüglich der Augen und den Anteilen bezüglich der Patienten in Abbildung 17 ergeben sich zum Beispiel aus Überschneidungen infolge von mehreren Komplikationsereignissen pro Auge oder pro Patient. So kam es zum Beispiel in sechs Fällen zum operationsbedingten Sekundärglaukom (15,2\%). Diese Operationen fanden jedoch an nur einem Auge (4,2\%) nur eines Patienten statt (5,3\%) (siehe auch Abbildung 18 - 20).

Abbildung 18 zeigt die Zahl der Komplikationen entsprechend der Art der Versorgung, Abbildung 19 die Zahl der komplikationsbehafteten Augen entsprechend der Versorgung und Abbildung 20 die Zahl der von Komplikationen betroffenen Patienten entsprechend der Versorgung.

In der Gruppe der aphak belassenen Patienten war die sekundäre Linsenkapselveränderung in zwei Fällen (20\%) die einzige Komplikation. Diese trat in zwei Augen (25\%) von zwei verschiedenen Patienten $(33,3 \%)$ auf.

In der Gruppe der primär versorgten Patienten waren sowohl einige Patienten als auch einige Augen mehrfach betroffen. Es kam zu fünf Re-Operationen (50\%) in drei Augen (37,5\%) von zwei Patienten $(33,3 \%)$ wegen sekundärer Linsenkapselveränderung. Außerdem wurden in einem Fall (20\%) bei einem Auge (20\%) eines Patienten (25\%) Synechien festgestellt.

In der Gruppe der sekundär versorgten Patienten kam es, als einzige Gruppe, in zehn Fällen von acht Augen bei sechs Patienten zur IOL-Luxation/Subluxation. Das heißt auch hier waren einige Augen und Patienten mehrfach betroffen. sekundäre Linsenkapselveränderungen traten in dieser Gruppe in drei Fällen (30\%) von drei Augen (37,5\%) zweier Patienten (33,3\%) auf. Synechien wurden in vier Fällen (80\%) von vier Augen (80\%) bei drei Patienten (75\%) festgestellt. Alle anderen Komplikationen, mit Ausnahme des Sekundärglaukoms (siehe oben), betrafen einen Fall in einem Auge eines Patienten der Gruppe der sekundär implantierten Patienten.

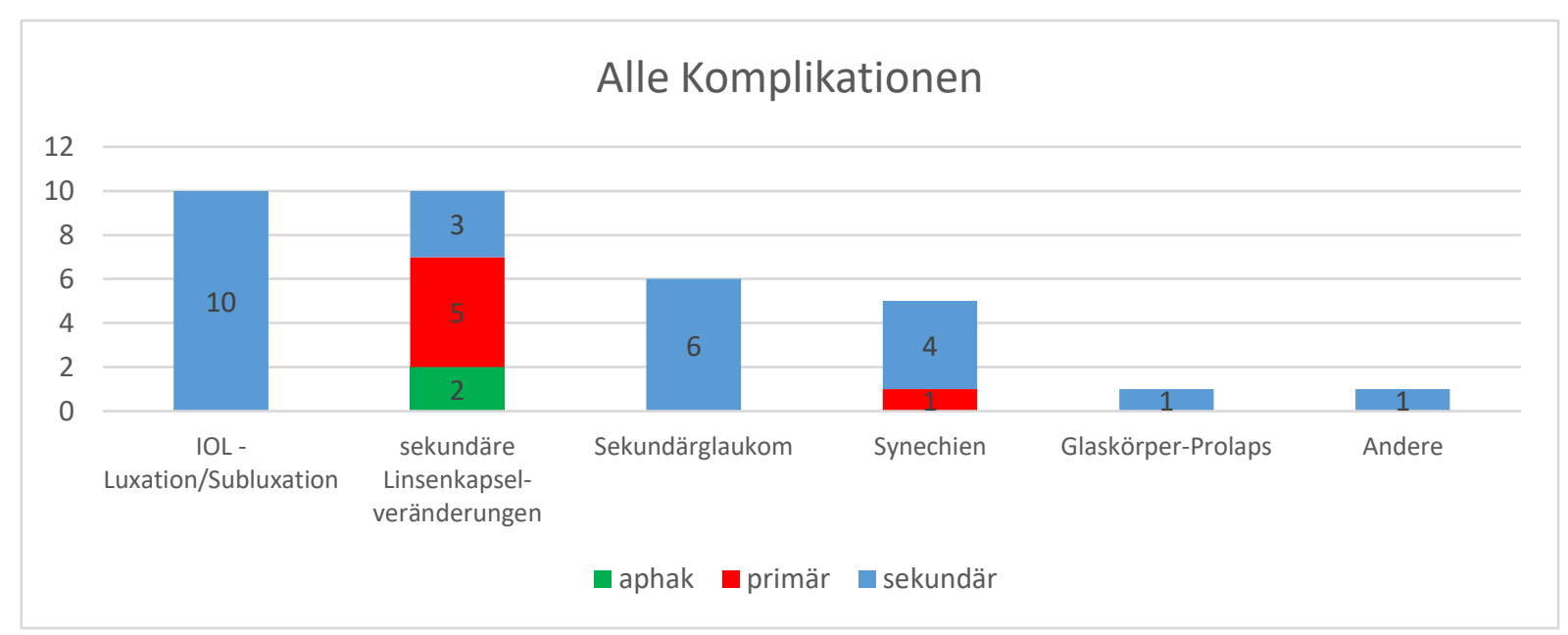

Abbildung 18 Verteilung aller Komplikationen bezüglich des Versorgungstyps 


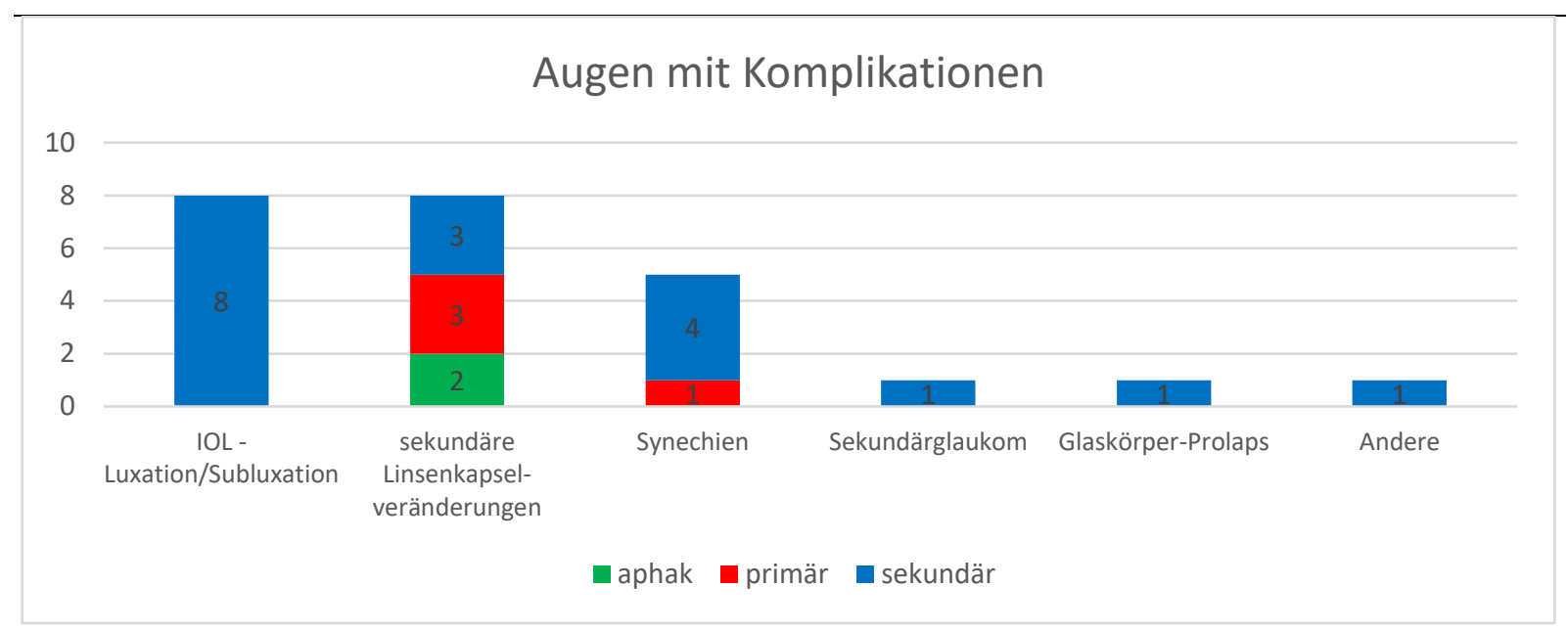

Abbildung 19 Verteilung aller komplikationsbehafteten Augen bezüglich des Versorgungstyps

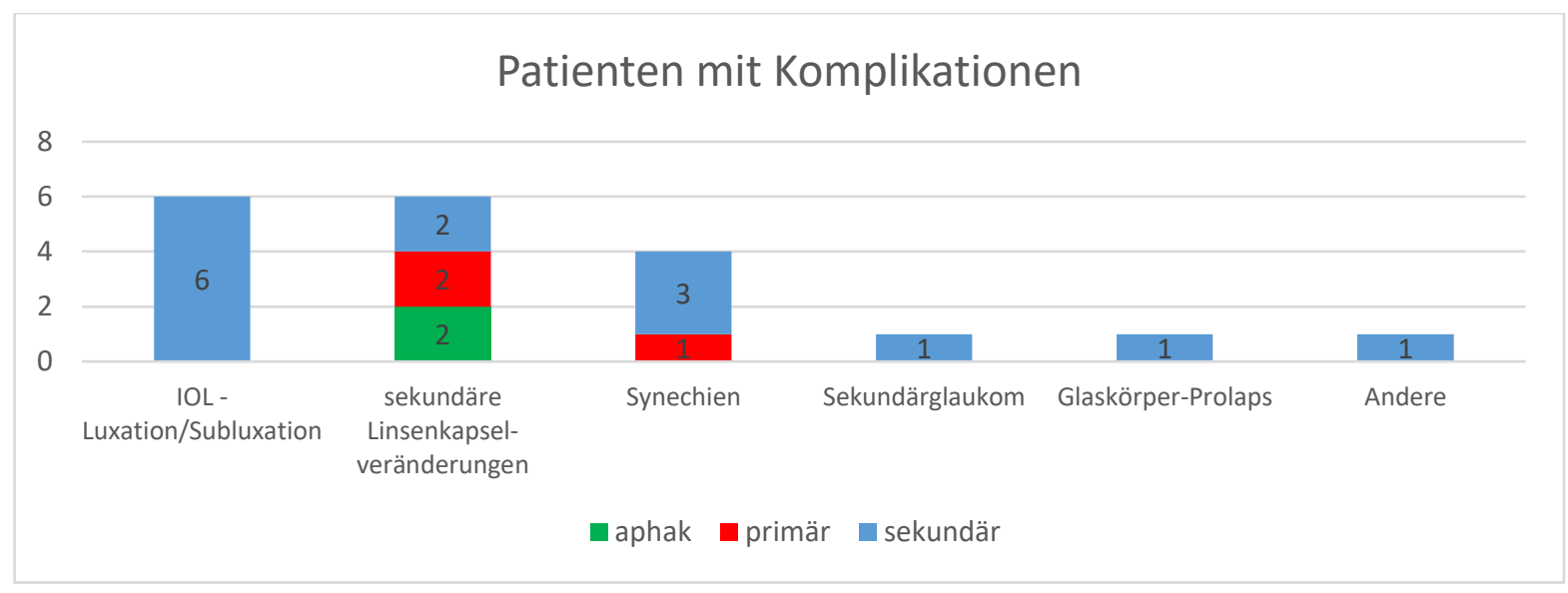

Abbildung 20 Verteilung aller von Komplikationen betroffenen Patienten bezüglich des Versorgungstyps

Auffällig ist, dass die IOL-Luxation/-Subluxation ausschließlich in der Gruppe der sekundärimplantierten Patienten auftritt. Außerdem ist zu bemerken, dass im Falle von sekundären Linsenkapselveränderungen und der IOL-Luxation/-Subluxation Patienten und Augen, die eine Komplikation erfahren haben, in unserer Untersuchung zum Teil erneut komplikationsbedingt reoperiert werden mussten (siehe Tabelle $6-8$ ). Dies zeigt sich insbesondere auch im Fall des Sekundärglaukoms.

Die Verteilung der aus den Komplikationen resultierenden Re-Operationen zeigt Tabelle 8. 
Tabelle 8 Verteilung der aus den Komplikationen resultierenden Re-Operationen nach Versorgungstyp

\begin{tabular}{|c|c|c|c|c|}
\hline \multirow[b]{2}{*}{ Erforderliche Re-Operation } & \multicolumn{3}{|c|}{ IOL } & \multirow[b]{2}{*}{$\begin{array}{l}\text { Total } \\
(n=74)\end{array}$} \\
\hline & $\begin{array}{l}\text { aphak } \\
(n=21)\end{array}$ & $\begin{array}{c}\text { Primär } \\
(n=5)\end{array}$ & $\begin{array}{c}\text { Sekundär } \\
(n=48)\end{array}$ & \\
\hline HKL-Einnähung / Austausch & 0 & 0 & 8 & $8(24,2 \%)$ \\
\hline HKL-Reposition & 0 & 0 & 2 & $2(6,1 \%)$ \\
\hline Nachstarentfernung & 2 & 5 & 3 & $10(30,3 \%)$ \\
\hline Synechiolyse & 0 & 1 & 4 & $5(15,2 \%)$ \\
\hline Vitrektomie & 0 & 0 & 1 & $1(3 \%)$ \\
\hline Zyklophotokoagulation (CPC) & 0 & 0 & 3 & $3(9,1 \%)$ \\
\hline andere & 0 & 0 & 1 & $1(3 \%)$ \\
\hline filtrierende Glaukom-OP & 0 & 0 & 3 & $3(9,1 \%)$ \\
\hline Total & $2(6.1 \%)$ & $6(18.2 \%)$ & $25(75.8 \%)$ & $33(100 \%)$ \\
\hline
\end{tabular}

Entsprechend den Komplikationen reduziert sich durch mehrfaches Auftreten bei einzelnen Augen und Patienten die Zahl der Nachstarentfernung je nach Betrachtungsweise (zehn Operationen in acht Augen bei sechs Patienten). Ebenso verhält es sich mit der HKLEinnähung/Austausch (acht Operationen in sieben Augen bei fünf Patienten). Die Zahl der Repositionen bleibt dabei gleich (je zwei). Es stellt sich heraus, dass an dem Auge mit dem Sekundärglaukom mehrere druckreduzierende Operationen durchgeführt werden mussten.

Tabelle 9 und Tabelle 10 zeigen das Patientenalter zum Zeitpunkt der Komplikation bezüglich der beiden untersuchten Faktoren in Jahren.

Tabelle 9 Patientenalter beim Auftreten der Komplikation in Jahren nach Versorgungstyp

\begin{tabular}{|l|r|r|c|}
\hline \multicolumn{1}{|c|}{ IOL } & N & Mean \pm SD & $95 \% \mathbf{C l}$ \\
\hline aphak & 2 & $0,50 \pm 0,24$ & $-1,69-2,69$ \\
\hline primär & 6 & $0,78 \pm 0,21$ & $0,56-1,00$ \\
\hline sekundär & 25 & $5,20 \pm 2,92$ & $4,00-6,41$ \\
\hline Total & 33 & $4,11 \pm 3,20$ & $2,98-5,25$ \\
\hline
\end{tabular}

Tabelle 10 Patientenalter beim Auftreten der Komplikation in Jahren nach Lateralität

\begin{tabular}{|l|c|c|c|}
\hline $\begin{array}{c}\text { Uni- } \\
\text { /bilateral }\end{array}$ & $\mathbf{N}$ & Mean \pm SD & \multicolumn{9}{c}{$\mathbf{9 5 \%} \mathbf{~ C l}$} \\
\hline bilateral & 21 & $3,31 \pm 2,73$ & $2,06-4,55$ \\
\hline unilateral & 12 & $5,53 \pm 3,58$ & $3,26-7,80$ \\
\hline Total & 33 & $4,11 \pm 3,20$ & $2,98-5,25$ \\
\hline
\end{tabular}


Es fällt auf, dass während die Kinder der aphak und primär versorgten Gruppe im Mittel im frühen Lebensalter Komplikationen erfahren, sich das Mittel bei den Sekundär-Implantierten weit nach hinten verschiebt. Entsprechendes zeigt sich auch in der Untersuchung des Zeitraums von der Kataraktextraktion bis zur Komplikation. Ebenso weist der Vergleich der bi- und unilateralen Patienten jeweils einen Unterschied auf.

Eine arbiträre Grenzsetzung nach dem ersten Lebensjahr deckt auf, dass die Mehrheit der Komplikationen nach dem ersten Lebensjahr aufgetreten sind (30,3\% vs. 69,7\%). Dies speist sich jedoch vor allem aus dem großen Anteil der Komplikationen in der sekundär versorgten Gruppe(> 1. Lj.: aphak 0\% (0 Kompl.), primär 33,3\% (2 Kompl.), sekundär 84\% (21 Kompl.)).

Im Vergleich des Zeitraums zwischen der Linsenimplantation und der Komplikation nähern sich die Unterschiede, wie in Tabelle 11 und 12 ersichtlich, an.

Tabelle 11 Zeitraum Linsenimplantation bis Komplikation in Jahren nach Versorgungstyp

\begin{tabular}{|l|r|r|l|}
\hline \multicolumn{1}{|c|}{ IOL } & $\mathbf{N}$ & Mean \pm SD & \multicolumn{1}{c|}{$95 \% \mathbf{C l}$} \\
\hline primär & 6 & $0,35 \pm 0.22$ & $0,12-0,58$ \\
\hline sekundär & 25 & $1,51 \pm 3,03$ & $0,26-2,76$ \\
\hline Total & 31 & $1,29 \pm 2,75$ & $0,28-2,30$ \\
\hline
\end{tabular}

Tabelle 12 Zeitraum Linsenimplantation bis Komplikation in Jahren nach Lateralität

\begin{tabular}{|l|c|r|c|}
\hline $\begin{array}{c}\text { Uni- } \\
\text { /bilateral }\end{array}$ & $\mathbf{N}$ & Mean \pm SD & \multicolumn{1}{c|}{$\mathbf{9 5 \%} \mathbf{~ C l}$} \\
\hline bilateral & 20 & $1,02 \pm 2,60$ & $-0,20-2,24$ \\
\hline unilateral & 11 & $1,78 \pm 3,06$ & $-0,28-3,83$ \\
\hline Total & 31 & $1,29 \pm 2,75$ & $0,28-2,30$ \\
\hline
\end{tabular}

Den großen Einfluss der sekundär versorgten Patienten zeigt daraufhin die spezielle Untersuchung dieser Gruppe. Tabelle 13 beweist, dass ein großer Teil der Komplikationen erst nach der Linsenimplantation stattgefunden hat, was aufgrund des späten durchschnittlichen Linsenimplantationsalters die hohe Differenz erklären könnte.

Tabelle 13 Verteilung der gesamten Komplikationen bei sekundär versorgten Patienten vor/nach Linsenimplantation

\begin{tabular}{|c|c|c|c|c|c|c|c|}
\hline \multirow[b]{2}{*}{$\begin{array}{c}\text { nach / vor } \\
\text { Linsenimplantation }\end{array}$} & \multicolumn{6}{|c|}{ Komplikation } & \multirow[b]{2}{*}{ Total } \\
\hline & Andere & $\begin{array}{c}\text { Glaskörper- } \\
\text { Prolaps }\end{array}$ & $\begin{array}{c}\text { IOL - Luxation/ } \\
\text { Subluxation }\end{array}$ & $\begin{array}{c}\text { Sekundäre } \\
\text { Linsenkapsel- } \\
\text { veränderung }\end{array}$ & $\begin{array}{l}\text { Sekundär- } \\
\text { glaukom }\end{array}$ & Synechien & \\
\hline nach & 1 & 0 & 10 & 0 & 6 & 3 & $20(80 \%)$ \\
\hline Total & $1(4 \%)$ & $1(4 \%)$ & $10(40 \%)$ & $3(12 \%)$ & $6(24 \%)$ & $4(16 \%)$ & $25(100 \%)$ \\
\hline
\end{tabular}


Auffällig ist, wie schon in den vorangegangenen Untersuchungen, der hohe Anteil an IOLLuxation/-Subluxation. Dieser sorgt für den Eindruck, dass Komplikationen mehrheitlich nach der IOL-Implantation auftreten. Verstärkt wird dies durch die sechs Behandlungen des Sekundärglaukoms, welche jedoch nur ein Auge betrafen.. Eine Untersuchung bezüglich der Augen ergibt ein ähnliches, nur leicht verändertes Bild (Tabelle 14).

Tabelle 14 Verteilung der Augen mit Komplikationen bei sekundär versorgten Patienten vor/nach Linsenimplantation

\begin{tabular}{|c|c|c|c|c|c|c|c|}
\hline \multirow[b]{2}{*}{$\begin{array}{c}\text { nach / vor } \\
\text { Linsenimplantation }\end{array}$} & \multicolumn{6}{|c|}{ Komplikation } & \multirow[b]{2}{*}{ Total } \\
\hline & Andere & $\begin{array}{l}\text { Glaskörper- } \\
\text { Prolaps }\end{array}$ & $\begin{array}{l}\text { IOL - Luxation/ } \\
\text { Subluxation }\end{array}$ & $\begin{array}{c}\text { Sekundäre } \\
\text { Linsenkapsel- } \\
\text { veränderung }\end{array}$ & $\begin{array}{l}\text { Sekundär- } \\
\text { glaukom }\end{array}$ & Synechien & \\
\hline nach & 1 & 0 & 8 & 0 & 1 & 3 & $13(72,2 \%)$ \\
\hline vor & 0 & 1 & 0 & 3 & 0 & 1 & $5(27,8 \%)$ \\
\hline Total & $1(5,6 \%)$ & $1(5,6 \%)$ & $8(44,4 \%)$ & $3(16,7 \%)$ & $1(5,6 \%)$ & $4(22,2 \%)$ & $18(100 \%)$ \\
\hline
\end{tabular}

\subsection{Bulbuslänge}

Zu Beginn der Untersuchung der Bulbuslänge war von Interesse, wie hoch der Anteil an Patienten mit Mikrophthalmus ist. Als Richtwert wurde eine Bulbuslänge in der ersten Messung kleiner als 15,5 mm gewählt. Insgesamt fielen sechs Patienten (8,1\%) unter diese Definition (primär 20\%, sekundär 6,3\%, aphak 9,5\%).

Die folgenden Ergebnisse schließen Patienten mit der Komplikation Sekundärglaukom aus. Der Versuch, Patienten mit Sekundärglaukom getrennt zu untersuchen, scheitert daran, dass nur ein Auge eines Patienten der Gesamtpopulation betroffen ist.

Tabelle 15, 16 und 17 gruppieren die Messungen in die Zeiträume null bis zwei Lebensjahre, drei bis sechs Lebensjahre sowie sieben und mehr Lebensjahre. Dabei zeigen sie einen Vergleich nach Behandlungsverfahren, Seitigkeit und Geschlecht. Mehrere Messungen eines Auges im gleichen Zeitraum wurden gemittelt. 
Tabelle 15 Durchschnittliche Bulbuslänge in mm je Zeitraum nach Versorgungstyp

\begin{tabular}{|l|l|r|r|r|}
\hline $\begin{array}{c}\text { Zeitraum } \\
\text { (Lebensjahr) }\end{array}$ & \multicolumn{1}{|c|}{ IOL } & N & Mean \pm SD & \multicolumn{1}{c|}{$95 \%$ CI } \\
\hline Geburt - 2tes & aphak & 19 & $18,63 \pm 2,61$ & $17,37-19,89$ \\
\hline & primär & 5 & $18,24 \pm 2,40$ & $15,26-21,22$ \\
\hline & sekundär & 43 & $18,45 \pm 1,74$ & $17,92-18,99$ \\
\hline 3tes - 7tes & Total & 67 & $18,49 \pm 2,04$ & $17,99-18,99$ \\
\hline & aphak & 13 & $20,58 \pm 3,20$ & $18,65-22,51$ \\
\hline & primär & 3 & $22,13 \pm 2,08$ & $16,95-27,30$ \\
\hline stes und älter & sekundär & 42 & $20,18 \pm 1,77$ & $19,63-20,74$ \\
\hline & Total & 58 & $20,37 \pm 2,18$ & $19,80-20,95$ \\
\hline & sekundär & 14 & $22,06 \pm 2,80$ & $20,44-23,67$ \\
\hline & Total & 23 & $21,64 \pm 2,78$ & $20,43-22,84$ \\
\hline & & & $20,99 \pm 2,79$ & $18,84-23,13$ \\
\hline
\end{tabular}

Deskriptiv kann der Eindruck gewonnen werden, dass im Zeitraum drei bis sechs Lebensjahre die Patienten mit primärer Versorgung einen größeren Bulbusdurchmesser aufweisen. Der paarweise t-Test der Gruppen weist in keinem Zeitraum einen signifikanten Unterschied nach.

Tabelle 16 Durchschnittliche Bulbuslänge in mm je Zeitraum nach Lateralität

\begin{tabular}{|l|l|l|l|l|}
\hline $\begin{array}{c}\text { Zeitraum } \\
\text { (Lebensjahr) }\end{array}$ & $\begin{array}{l}\text { Betroffene } \\
\text { Seite }\end{array}$ & $\mathbf{N}$ & Mean \pm SD & $\begin{array}{c}\text { Mean } \\
\text { (95\%UCL) }\end{array}$ \\
\hline Geburt - 2tes & bilateral & 36 & $17,70 \pm 1,84$ & $17,08-18,32$ \\
\hline & unilateral & 31 & $19,40 \pm 1,90$ & $18,71-20,10$ \\
\hline 3tes - 6tes & Total & 67 & $18,49 \pm 2,04$ & $17,99-18,99$ \\
\hline & bilateral & 32 & $19,66 \pm 1,76$ & $19,03-20,30$ \\
\hline utes \& älter & unilateral & 26 & $21,24 \pm 2,37$ & $20,29-22,20$ \\
\hline & Total & 58 & $20,37 \pm 2,18$ & $19,80-20,95$ \\
& unilateral & 16 & $20,66 \pm 1,89$ & $19,66-21,67$ \\
\hline & Total & 23 & $21,64 \pm 2,78$ & $20,43-22,84$ \\
\hline
\end{tabular}

Im Vergleich der Seitigkeit scheint es, dass Patienten mit unilateraler Katarakt einen durchgehend größeren Bulbusdurchmesser haben. Im paarweisen t-Test zeigt sich bei einem Signifikanzniveau von 5\% in allen Abschnitten ein signifikanter Unterschied bezüglich der betroffenen Seite (Geburt - zweites $p=0,0004$; drittes - sechstes Lj. $p=0,0051$; siebtes Lj. \& älter $p=0,0077$ ). 


\begin{tabular}{|l|l|l|r|r|}
\hline $\begin{array}{c}\text { Zeitraum } \\
\text { (Lebensjahr) }\end{array}$ & Geschlecht & N & Mean \pm SD & Mean (95\%UCL) \\
\hline Geburt - 2tes & männlich & 38 & $18,97 \pm 1,80$ & $18,38-19,56$ \\
\hline & weiblich & 29 & $17,86 \pm 2,19$ & $17,02-18,69$ \\
\hline Total & 67 & $18,49 \pm 2,04$ & $17,99-18,99$ \\
\hline 3tes - 6tes & männlich & 33 & $20,81 \pm 2,18$ & $20,04-21,59$ \\
\hline & weiblich & 25 & $19,79 \pm 2,09$ & $18,93-20,65$ \\
\hline 7tes \& älter & motal & 58 & $20,37 \pm 2,18$ & $19,80-20,95$ \\
\hline & männlich & 15 & $22,22 \pm 2,85$ & $20,64-23,80$ \\
\hline & Toiblich & 8 & $20,54 \pm 2,44$ & $18,51-22,58$ \\
\hline
\end{tabular}

Auch im Vergleich der Geschlechter scheinen männliche Patienten einen längeren Bulbus aufzuweisen. Dieser Unterschied vergrößert sich im Verlauf der Zeiträume. Im paarweisen t-Test lässt sich bei einem Signifikanzniveau von 5\% jedoch nur im Zeitraum von der Geburt bis zweites Lebensjahren ein signifikanter Unterschied ausmachen $(p=0,0257)$. Die Gruppe zweites bis sechstes Lebensjahr weist einen Wert von $\mathrm{p}=0,0778$ und die Gruppe siebtes Lebensjahr und älter einen Wert von $\mathrm{p}=0,174$ auf.

Eine alleinstehende Untersuchung der Patienten mit der Komplikation sekundäre Linsenkapselveränderung ergibt das Bild in Tabelle 18.

Tabelle 18 Durchschnittliche Bulbuslänge in mm je Zeitraum bei Patienten mit der Komplikation sekundäre Linsenkapselveränderung nach Versorgungstyp

\begin{tabular}{|l|l|r|r|r|}
\hline $\begin{array}{c}\text { Zeitraum } \\
\text { (Lebensjahr) }\end{array}$ & \multicolumn{1}{|c|}{ IOL } & N & \multicolumn{1}{c|}{ Mean \pm SD } & Mean (95\%UCL) \\
\hline Geburt - 2tes & aphak & 2 & $16,92 \pm 1,95$ & $-0,59-34,42$ \\
& primär & 3 & $16,82 \pm 1,17$ & $13,93-19,72$ \\
& sekundär & 2 & $17,63 \pm 0,04$ & $17,26-17,99$ \\
\hline Ttes -6tes & Total & 7 & $17,08 \pm 1,11$ & $16,06-18,10$ \\
\hline & aphak & 2 & $18,09 \pm 1,66$ & $3,20-32,98$ \\
\hline primär & 2 & $21,21 \pm 1,92$ & $3,96-38,46$ \\
\hline & sekundär & 3 & $19,08 \pm 0,81$ & $17,07-21,10$ \\
\hline & Total & 7 & $19,41 \pm 1,73$ & $17,81-21,01$ \\
\hline & aphak & 2 & $19,06 \pm 1,25$ & $7,81-30,30$ \\
\hline & sekundär & 1 & 21,17 & \\
\hline & Total & 3 & $19,76 \pm 1,51$ & $16,01-23,51$ \\
\hline
\end{tabular}

Um zu ermitteln, ob sich die Komplikation auf das Bulbuswachstum auswirkt, erfolgte ein Vergleich der Gruppen mit und ohne sekundäre Linsenkapselveränderung. Im Zeitraum bis Ende 
des zweiten Lebensjahres sind die Mittelwerte dabei an der Grenze zu signifikant kleiner $(p=0,0527)$. In den beiden folgenden Zeiträumen wiederholt sich dieses Ergebnis nicht.

\subsubsection{Rate of Axial Growth (RAG)}

Patienten mit der Komplikation Sekundärglaukom (ein Auge) und mit sekundären Linsenkapselveränderungen (vier Augen) wurden nicht einbezogen, um diese gesondert zu vergleichen. In der Gruppe der Patienten mit Sekundärglaukom war dies aus Mangel an Augen nicht möglich. Zur Untersuchung der RAG wurde zuerst eine Baseline +/- eine Woche zur Kataraktextraktion gebildet. Insgesamt konnte bei 46 Augen eine Baseline gebildet werden. Außerdem wurde ein Mindest-Nachuntersuchungszeitraum von zwei Jahren definiert. Insgesamt erfüllten 33 Augen diese Bedingung. Die Schnittmenge dieser beiden Gruppen umfasste 33 Augen (aphak 18,2\%, primär 3\%, sekundär 78,78\%).

Der durchschnittliche Nachuntersuchungszeitraum als Zeitraum zwischen Baseline-Untersuchung und letzter Untersuchung ist in Tabelle 19 einsehbar und betrug insgesamt ca. 5,9 Jahre. Die Messung der primär versorgten Patienten wurde aus Mangel an Augen von der weiteren Untersuchung ausgenommen.

Tabelle 19 Durchschnittlicher Nachuntersuchungszeitraum in Tagen

\begin{tabular}{|lr|r|c|}
\hline \multicolumn{1}{|c|}{ IOL } & N & \multicolumn{1}{c|}{ Mean \pm SD } & \multicolumn{1}{c|}{$95 \% \mathbf{C l}$} \\
\hline aphak & 6 & $1640,00 \pm 711,72$ & $893,09-2386,91$ \\
\hline primär & 1 & 1342,00 & \\
sekundär & 26 & $2284,12 \pm 1524,86$ & $1668,21-2900,02$ \\
\hline Total & 33 & $2138,45 \pm 1406,89$ & $1639,59-2637,32$ \\
\hline
\end{tabular}

Ein Vergleich zeigte keinen signifikanten Unterschied zwischen den Gruppen (aphak/sekundär, unilateral/bilateral, sekundäre Linsenkapselveränderung ja/nein) in Bezug auf den Nachuntersuchungszeitraum.

Abbildung 21 zeigt die Entwicklung der Bulbuslänge. 


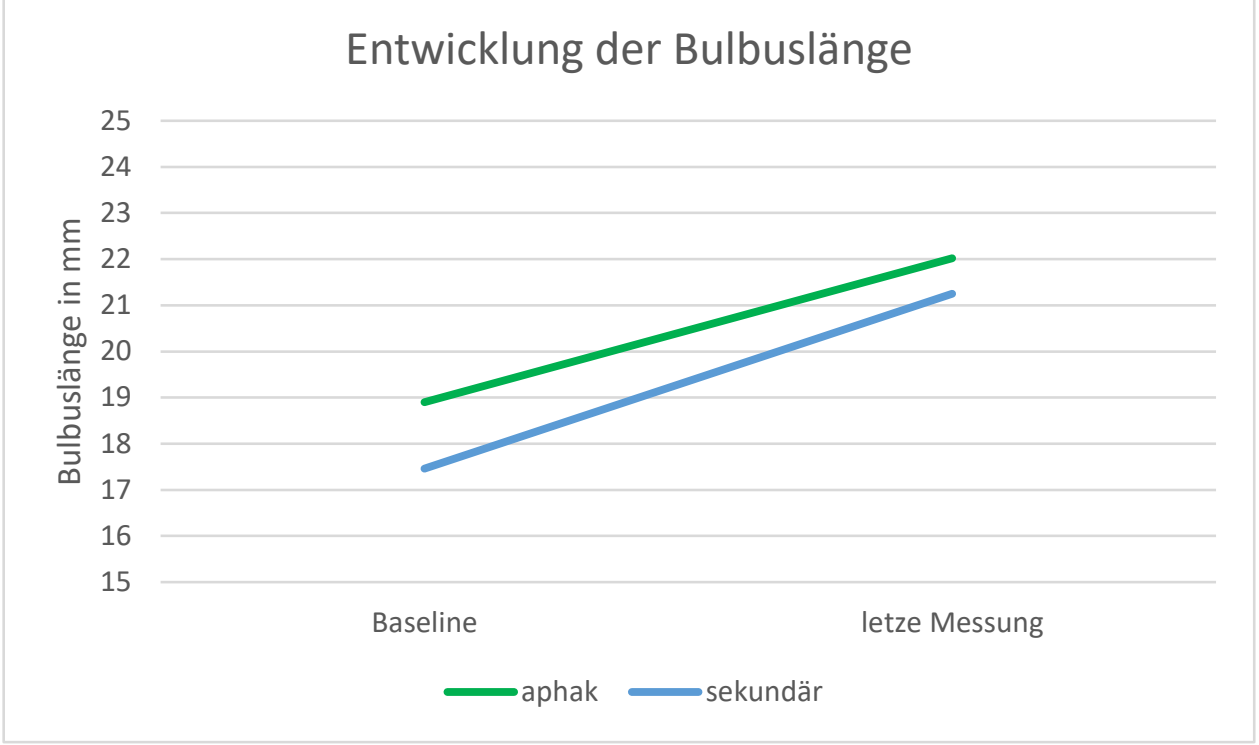

Abbildung 21 Entwicklung der Bulbuslänge zwischen Baseline und letzter Messung nach Versorgungstyp

Tabelle 20 zeigt die durchschnittliche Bulbuslänge zur Baseline-Messung. Der Vergleich der Gruppen (aphak/sekundär, unilateral/bilateral, sekundäre Linsenkapselveränderung ja/nein) weist einen signifikanten Unterschied in der Bulbuslänge bezüglich der Lateralität (bilateral 16,91 +/$0,37 \mathrm{~mm}$ vs. unilateral 18,58 +/-0,42 mm, $\mathrm{p}=0,0064)$ und der Patienten mit und ohne sekundäre Linsenkapselveränderung auf (mit 16,26 +/- 0,09 mm vs. ohne 17,82 +/- 0,32 mm, p<0,0001).

Tabelle 20 Bulbuslänge in mm zur Baseline-Untersuchung

\begin{tabular}{|l|r|r|r|}
\hline \multicolumn{1}{|c|}{ IOL } & \multicolumn{1}{c|}{$\mathbf{N}$} & \multicolumn{1}{c|}{ Mean \pm SD } & \multicolumn{1}{l|}{$95 \% \mathbf{C l}$} \\
\hline aphak & 6 & $18,89 \pm 2,62$ & $16,14-21,63$ \\
\hline primär & 1 & 20,70 & \\
\hline sekundär & 26 & $17,46 \pm 1,45$ & $16,87-18,05$ \\
\hline Total & 33 & $17,82 \pm 1,82$ & $17,17-18,46$ \\
\hline
\end{tabular}

Tabelle 21 zeigt die Verteilung zum Zeitpunkt der letzten Messung. Der Vergleich der Gruppen (aphak/sekundär, unilateral/bilateral, sekundäre Linsenkapselveränderung ja/nein) weist nur noch im Vergleich der Lateralität auf einen Unterschied hin (bilateral 20,51 +/- 0,58 mm vs. unilateral $22,27+/-0,6 \mathrm{~mm}, \mathrm{p}=0,0457)$.

Tabelle 21 Bulbuslänge im mm zum Zeitpunkt der letzten Messung nach Versorgungstyp

\begin{tabular}{|l|r|r|r|}
\hline \multicolumn{1}{|c|}{ IOL } & \multicolumn{1}{c|}{ N } & \multicolumn{1}{c|}{ Mean \pm SD } & \multicolumn{1}{l|}{$95 \% \mathbf{C l}$} \\
\hline aphak & 6 & $22,02 \pm 3,88$ & $17,95-26,10$ \\
\hline primär & 1 & 23,95 & \\
\hline sekundär & 26 & $21,25 \pm 2,21$ & $20,35-22,14$ \\
\hline Total & 33 & $21,47 \pm 2,54$ & $20,57-22,37$ \\
\hline
\end{tabular}


Das Bulbuswachstum als Differenz der Bulbuslänge zur letzten Messung und der Bulbuslänge zur Baseline zeigt Tabelle 22. Es kann kein statistisch signifikanter Unterschied zwischen den Gruppen (aphak/sekundär, unilateral/bilateral, sekundäre Linsenkapselveränderung ja/nein) nachgewiesen werden.

Tabelle 22 Bulbuswachstum in mm nach Versorgungstyp

\begin{tabular}{|l|r|r|r|}
\hline \multicolumn{1}{|c|}{ IOL } & \multicolumn{1}{c|}{ N } & \multicolumn{1}{c|}{ Mean \pm SD } & \multicolumn{1}{c|}{$95 \% \mathbf{C l}$} \\
\hline aphak & 6 & $3,14 \pm 1,92$ & $1,12-5,15$ \\
primär & 1 & 3,25 & \\
sekundär & 26 & $3,79 \pm 1,78$ & $3,07-4,51$ \\
\hline Total & 33 & $3,65 \pm 1,77$ & $3,03-4,28$ \\
\hline
\end{tabular}

Die rate of axial growth (RAG) zeigt bezüglich der Linsenversorgung die Tabelle 23. Auch in dieser Gruppe konnte zwischen den Gruppen (aphak/sekundär, unilateral/bilateral, sekundäre Linsenkapselveränderung ja/nein) kein signifikanter Unterschied nachgewiesen werden.

Tabelle 23 Rate of axial growth nach Versorgungstyp

\begin{tabular}{|l|r|r|r|}
\hline \multicolumn{1}{|c|}{ PSK } & N & \multicolumn{1}{c|}{ Mean \pm SD } & \multicolumn{1}{c|}{$95 \% \mathbf{C l}$} \\
\hline aphak & 6 & $16,60 \pm 10,16$ & $5,94-27,26$ \\
\hline primär & 1 & 15,70 & \\
\hline sekundär & 26 & $21,69 \pm 10,20$ & $17,57-25,81$ \\
\hline Total & 33 & $20,58 \pm 10,10$ & $17,00-24,16$ \\
\hline
\end{tabular}

\subsection{Sphärisches Äquivalent}

Die durchschnittliche Dauer zwischen Kataraktextraktion und letzter möglicher Refraktionsmessung bezüglich des Versorgungstyps, der Lateralität und dem Auftreten von Komplikationen sind in Form des sphärischen Äquivalents in Tabelle 24, 25 und 26 dargestellt.

Tabelle 24 Zeitraum Kataraktextraktion bis letztmögliche Refraktionsmessung nach Versorgungstyp in Jahren

\begin{tabular}{|l|r|r|r|}
\hline \multicolumn{1}{|c|}{ IOL } & \multicolumn{1}{c|}{$\mathbf{N}$} & \multicolumn{1}{c|}{ Mean \pm SD } & \multicolumn{1}{c|}{$95 \% \mathbf{~ C l}$} \\
\hline aphak & \multicolumn{1}{c|}{17} & $5,64 \pm 3,44$ & $3,88-7,41$ \\
\hline primär & 4 & $5,93 \pm 3,67$ & $0,08-11,77$ \\
\hline sekundär & 47 & $7,55 \pm 3,29$ & $6,58-8,51$ \\
\hline Total & 68 & $6,97 \pm 3,41$ & $6,15-7,80$ \\
\hline
\end{tabular}


Tabelle 25 Zeitraum Kataraktextraktion bis letztmögliche Refraktionsmessung nach Lateralität in Jahren

\begin{tabular}{|l|r|r|r|}
\hline $\begin{array}{c}\text { uni- } \\
\text { /bilateral }\end{array}$ & N & Mean \pm SD & Mean (95\%UCL) \\
\hline bilateral & 38 & $7,76 \pm 3,62$ & $6,57-8,95$ \\
\hline unilateral & 30 & $5,98 \pm 2,87$ & $4,91-7,05$ \\
\hline Total & 68 & $6,97 \pm 3,41$ & $6,15-7,80$ \\
\hline
\end{tabular}

Tabelle 26 Zeitraum Kataraktextraktion bis letztmögliche Refraktionsmessung nach Komplikation ja/nein in Jahren

\begin{tabular}{|l|l|r|r|}
\hline Komplikation & $\mathbf{N}$ & Mean \pm SD & Mean (95\%UCL) \\
\hline ja & 13 & $7,64 \pm 2,78$ & $5,96-9,32$ \\
\hline nein & 55 & $6,82 \pm 3,54$ & $5,86-7,78$ \\
\hline Total & 68 & $6,97 \pm 3,41$ & $6,15-7,80$ \\
\hline
\end{tabular}

Aus den vorangegangenen Kapiteln ist zu erkennen, dass die sekundär versorgten Patienten einen längeren Nachuntersuchungszeitraum aufweisen. Ebenso verhält es sich bei Patienten mit bilateraler Katarakt.

Eine Gruppierung bezüglich des Lebensalters zur letzten Refraktionsmessung und eine Mittelung des entsprechenden sphärischen Äquivalents zeigt bezüglich der Zeiträume vom dritten bis sechsten Lebensjahr und vom siebten Lebensjahr und älter die Tabelle 27. Ein Vergleich der Versorgungstypen zeigt im paarweisen t-Test bezüglich des Zeitraums von sieben Lebensjahren und älter jeweils einen signifikanten Unterschied $(\mathrm{p}<0,0001)$ bei $5 \%$ Signifikanzniveau.

Tabelle 27 Durchschnittliches sphärisches Äquivalent in dpt je Zeitraum nach Versorgungstyp

\begin{tabular}{|llr|r|r|}
\hline $\begin{array}{c}\text { Zeitraum } \\
\text { (Lebensjahr) }\end{array}$ & \multicolumn{1}{|c|}{ PSK } & N & Mean \pm SD & \multicolumn{1}{c|}{$95 \%$ Cl } \\
\hline 3tes -6tes & aphak & 2 & $14,50 \pm 3,54$ & $-17,27-46,27$ \\
\hline & primär & 2 & $-1,00 \pm 8,49$ & $-77,24-75,24$ \\
\hline & sekundär & 11 & $2,80 \pm 10,15$ & $-4,02-9,61$ \\
\hline & Total & 15 & $3,85 \pm 10,00$ & $-1,69-9,39$ \\
\hline 7tes \& älter & aphak & 10 & $15,33 \pm 5,73$ & $11,22-19,43$ \\
& primär & 2 & $-12,50 \pm 3,54$ & $-44,27-19,27$ \\
\hline & sekundär & 36 & $-0,82 \pm 2,40$ & $-1,63--0,01$ \\
\hline & Total & 48 & $2,06 \pm 7,98$ & $-0,26-4,37$ \\
\hline
\end{tabular}

Die Verteilung entsprechend der Seitigkeit und des Auftretens von Komplikationen zeigen Tabelle 28 und 29. 
Tabelle 28 Durchschnittliches sphärisches Äquivalent in dpt je Zeitraum nach Lateralität

\begin{tabular}{|ll|r|r|r|}
\hline $\begin{array}{c}\text { Zeitraum } \\
\text { (Lebensjahr) }\end{array}$ & $\begin{array}{c}\text { Uni- } \\
\text { /bilateral }\end{array}$ & N & Mean \pm SD & \multicolumn{1}{c|}{$\begin{array}{c}\text { Mean } \\
(95 \% \text { UCL) }\end{array}$} \\
\hline 3tes -6tes & bilateral & 4 & $8,56 \pm 16,37$ & $-17,48-34,60$ \\
\hline & unilateral & 11 & $2,14 \pm 6,90$ & $-2,50-6,77$ \\
\hline & Total & 15 & $3,85 \pm 10,00$ & $-1,69-9,39$ \\
\hline 7tes \& älter & bilateral & 30 & $1,91 \pm 8,73$ & $-1,35-5,17$ \\
\hline & unilateral & 18 & $2,31 \pm 6,78$ & $-1,07-5,68$ \\
\hline & Total & 48 & $2,06 \pm 7,98$ & $-0,26-4,37$ \\
\hline
\end{tabular}

Tabelle 29 Durchschnittliches sphärisches Äquivalent in dpt je Zeitraum nach Komplikation ja/nein

\begin{tabular}{|l|l|r|r|r|}
\hline $\begin{array}{c}\text { Zeitraum } \\
\text { (Lebensjahr) }\end{array}$ & Komplikation & $\mathbf{N}$ & \multicolumn{1}{c|}{ Mean $\mathbf{~ S D ~}$} & \multicolumn{1}{c|}{$\mathbf{9 5 \%} \mathbf{~ C l}$} \\
\hline 3tes - 6tes & ja & 2 & $2,50 \pm 3,54$ & $-29,27-34,27$ \\
\hline & nein & 13 & $4,06 \pm 10,74$ & $-2,43-10,55$ \\
\hline & Total & 15 & $3,85 \pm 10,00$ & $-1,69-9,39$ \\
\hline 7tes \& älter & ja & 11 & $-1,45 \pm 7,00$ & $-6,15-3,25$ \\
\hline & nein & 37 & $3,10 \pm 8,04$ & $0,42-5,78$ \\
\hline & Total & 48 & $2,06 \pm 7,98$ & $-0,26-4,37$ \\
\hline
\end{tabular}

Die Varianzanalyse des sphärischen Äquivalents unter gemeinsamer Berücksichtigung der Faktoren Alter zur Refraktionsmessung, Versorgungstyp, Lateralität, und dem Auftreten von Komplikationen sowie deren Wechselwirkung mit dem Alter zur Refraktionsmessung zeigt das Alter zur Refraktionsmessung $(\mathrm{F}(1,58)=10,10 ; \mathrm{p}=0,0024)$ und den Versorgungstyp $(\mathrm{F}(2,58)=9,64$; $\mathrm{p}=0,0002)$ als signifikante Einflussfaktoren. Der hier gezeigte Einfluss des Versorgungstyps auf das finale sphärische Äquivalent lässt sich schon im Scatterplot in Abbildung 10 durch den nahezu parallelen Verlauf der roten und grünen Linie erahnen. 
Seite 44

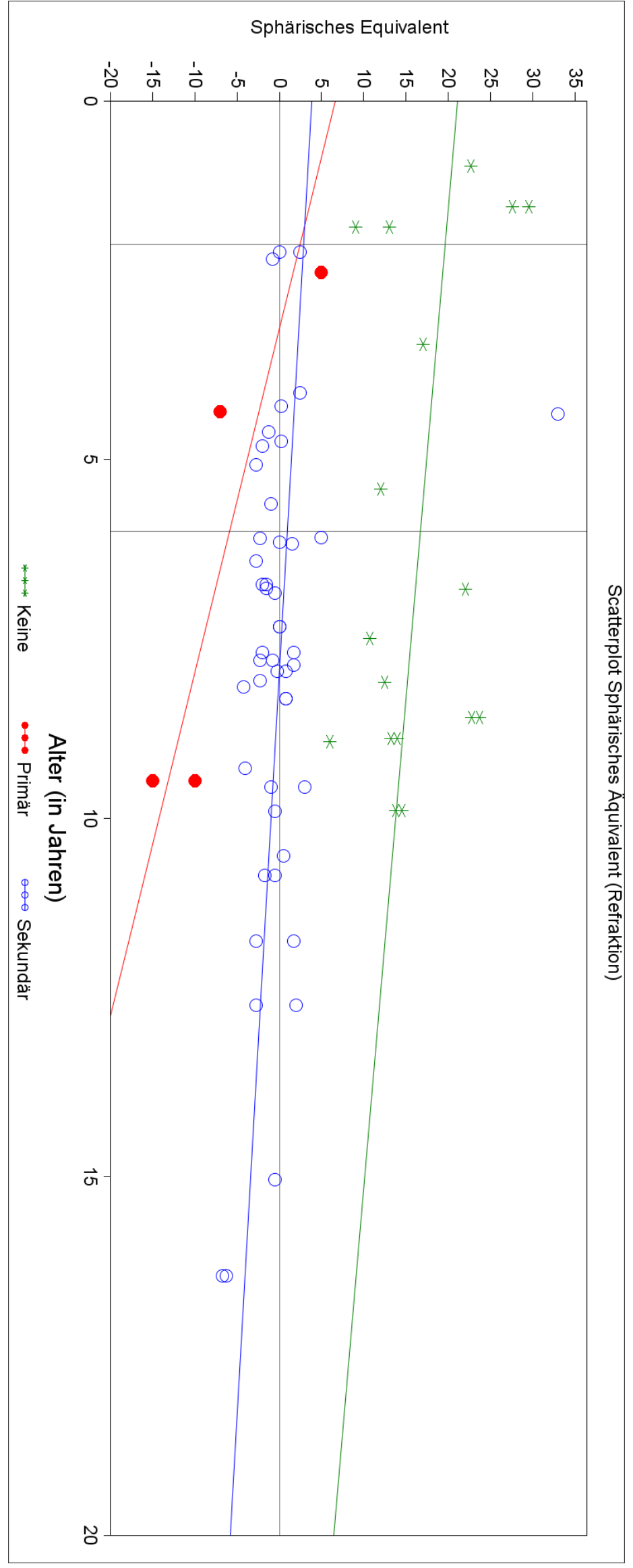

Abbildung 22 Verteilung des sphärischen Äquivalents bezügliche des Alters zum Messzeitpunkt nach Versorgungstyp 


\section{Diskussion}

Bei der chirurgischen Versorgung einer kongenitalen Katarakt gibt es noch immer keine klaren Leitlinien, es bleibt in der Regel eine Einzelfallentscheidung, ob man eine Kunstlinse implantiert oder das Auge zunächst linsenlos belässt und mit einer Kontaktlinse versorgt. Grundsätzlich gilt die Empfehlung, Kinder unter zwei Jahren linsenlos zu belassen, aber es gibt einige Situationen (z. B. fehlende Mitarbeit der Eltern beim Kontaktlinsenwechsel), bei denen eine primäre Linsenimplantation auch vor dem zweiten Lebensjahr gerechtfertigt ist. Der operative Eingriff unterscheidet sich von der Kataraktoperation beim Erwachsenen grundlegend, und auch die intraund postoperative Komplikationsrate ist unterschiedlich. In der vorliegenden Arbeit lag unser Fokus auf genau diesen intra- und postoperativen Komplikationen.

\subsection{Komplikationen}

Das Hauptergebnis der vorliegenden Arbeit lautet, dass das Auftreten von Komplikationen signifikant von der Art der Linsenversorgung abhängt ( $p=0,0423)$. Bei genauerer Betrachtung hält sich diese Aussage jedoch nur im Vergleich der aphaken Gruppe mit den primär implantierten Patienten $(p=0,0335)$. Der Vergleich zwischen primär und sekundär implantierten Patienten kommt zu keinem signifikanten Unterschied $(\mathrm{p}=0,0882)$, ebenso wie der Vergleich zwischen aphaken und sekundär implantierten Patienten $(\mathrm{p}=0$,2689). Hierbei ist aber zu bedenken, dass eine Komplikation im höheren Lebensalter aufgrund einer späteren IOL-Implantation sich weniger dramatisch auf die Sehentwicklung auswirkt als Komplikationen in früheren Lebensjahren. Es gibt zurzeit keine vergleichbaren Studien, die einen Unterschied zwischen allen drei Gruppen untersuchen.

Die IoLunder2-Studie (Solebo et al. 2015), welche primär implantierte und aphake Patienten über einen Nachuntersuchungszeitraum von zwölf Monaten verglichen hat, kam ähnlich wie die vorliegende Untersuchung $\mathrm{zu}$ dem Ergebnis, dass primär implantierte Augen mehr Komplikationen aufweisen. Dies gilt sowohl für die unilaterale (adjusted OR 16,7; 95\%CI 4,1 68,$9 ; \mathrm{p}<0,001$ ) als auch für die bilaterale Katarakt (adjusted OR 5,5;95\%CI 2,2 - 13,2; p<0,01). Die Autoren schließen daraus, dass die primäre IOL-Versorgung von Kindern mit kongenitaler Katarakt in Anbetracht des vergleichbaren funktionellen Ergebnisses und der höheren ReOperationsrate zurückhaltend eingesetzt werden sollte.

Kritik an dieser Aussage kam dagegen von den Autoren der IATS-Studie, welche primär implantierte und aphake Augen verglichen hat. Sie kamen zwar zu ähnlichen Ergebnissen wie die Autoren der Iolunder2-Studie bezüglich des funktionellen Ergebnisses und der Verteilung der ReOperationen in Fällen von unilateraler Katarakt in einem Nachuntersuchungszeitraum von fünf Jahren (Lambert et al. 2014; Plager et al. 2014). Die Autoren gaben jedoch zu bedenken, dass sich ein Großteil der aphaken beziehungsweise kontaktlinsenversorgten, Patienten zu einem späteren Zeitpunkt noch für die sekundäre IOL-Implantation entscheiden wird. Dies kann dann seinerseits zu weiteren Komplikationen führen die bei der Diskussion um die Komplikationsrate mit 
berücksichtigt werden müsste (Plager et al. 2014). Diese Datenlücke kann die vorliegende Untersuchung schließen, da auch sekundäre Linsenimplantationen miterfasst wurden. Unsere Daten legen nahe, dass sich ein Großteil der Komplikationen der sekundär implantierten Gruppe erst nach der Linsenimplantation offenbart (80\%). Allerdings sind die Kinder dann älter, die Augen oft schon ausgereift und die Entwicklung des visuellen Systems nahezu abgeschlossen, so dass Komplikationen in diesem Stadium anders gewichtet werden müssen als Komplikationen im ersten Lebensjahr. Auch andere Autoren kommen bei Kindern mit bilateraler Katarakt zu dem Schluss, dass es langfristig keinen Unterschied in der Häufigkeit von Komplikationen zwischen den primär und sekundär Versorgten gibt (Magli et al. 2013). Über die Qualität dieser Komplikationen und die Auswirkungen auf die visuelle Entwicklung wird aber nichts gesagt. Bei der Interpretation dieses Ergebnisses ist deshalb zu bedenken, dass ein späteres Komplikationsauftreten, zum Beispiel wie in unserem Fall nach der sekundären IOL-Implantation, zu einem insgesamt geringerem Amblyopierisiko dieser Gruppe führen könnte.

Es ergibt sich aus unseren Daten die Kritik, dass sich die sekundär implantierten Augen mit einem durchschnittlichen Nachuntersuchungszeitraum von 8,39 Jahren stark von der Gruppe der primär implantierten Augen unterscheidet. Dem steht entgegen, dass der Großteil der Komplikationen der sekundären Gruppe nach IOL-Implantation auftrat. Der Unterschied im Zeitraum zwischen IOL-Implantation und letzter Untersuchung ist jedoch vergleichsweise klein (primär 4,82 Jahre vs. sekundär 4,25 Jahre).

\subsubsection{IOL-Luxation/Subluxation}

Zu den zwei insgesamt am häufigsten aufgetretenen Komplikationen zählt die IOL-Luxation/Subluxation. Dies ist überraschend, scheint es doch in der Literatur eine vergleichsweise seltene Komplikation zu sein. So berichten Rong und Kollegen zum Beispiel über nur ein Kind mit IOLDislokation $(0,9 \%)$ in 110 sekundär implantierten Augen bei einem ähnlichen Nachuntersuchungszeitraum (rund 8,5 Jahren) (Rong et al. 2015) oder Michael Struck über nur einen Patienten mit Linsendislokation aus 14 Augen mit primärer IOL-Implantation, bei einem durchschnittlichen Nachbeobachtung von fünf Jahren (Struck 2015). In der vorliegenden Arbeit wurden Dislokationen ausschließlich in der Gruppe der sekundär implantierten Patienten beobachtet (16,6\% aus 48 sekundär implantierten Augen, 10,8\% aus allen untersuchten Augen). Die Ursachen einer IOL-Luxation oder Dislokation sind weit gefächert und unter anderem vom Ort der Implantation abhängig. Leider ist passende Literatur dazu insbesondere bei Kindern rar gesät, da es sich bei Linsendislokationen im Allgemeinen eher um eine mit niedrigem kumulativen Risiko versehene Spätfolge einer Linsenimplantation handelt (Pueringer et al. 2011). Unabhängig vom speziellen Thema der kongenitalen Katarakt finden sich als Ursachen für IOL-Luxationen und -Dislokationen - je nach Studie mit verschiedener Gewichtung - eine idiopathische Luxation, Traumata, das Pseudoexfoliationssyndrom, eine Uveitis, eine lange Achse oder Zonulolysen (Gross et al. 2004; Pueringer et al. 2011; Fujikawa et al. 2018). Hayashi und Kollegen untersuchten prädispositorische Faktoren für in-the-bag- und out-of-the-bag-Dislokationen. Dabei fanden sie die sekundäre IOL-Implantation $(45,8 \%)$ als häufigsten prädispositorischen Faktor bei out-of-the-bag- 
Dislokationen. Es folgen chirurgische Komplikationen (12,5\%) und reifer Katarakt (12,5\%) (Hayashi et al. 2007). In unserem Kollektiv wurden die Kunstlinsen nie in den Kapselsack gesetzt, da selbst bei einer primären Implantation dann ein später erforderlicher Austausch schwieriger wird. Leider lässt sich aus dem gegeben Datensatz weder die Art der Dislokation ermitteln noch womit die gegebene Häufigkeit zusammenhängen könnte.

\subsubsection{Sekundäre Linsenkapselveränderung}

Es besteht Konsens darüber, dass die sekundäre Linsenkapselveränderung oder die visual axis opacification (VAO) die häufigste Komplikation nach Kataraktextraktion bei kongenitaler Katarakt ist (Lu et al. 2010; Plager et al. 2014; Rong et al. 2015; Solebo et al. 2015). Dies deckt sich mit den Ergebnissen unserer Untersuchung, bei der sekundäre Linsenkapselveränderungen mit 33,3\% der aufgetretenen Komplikationen zu den beiden häufigsten Komplikationen gehörten. Dabei ist der Gesamtanteil an sekundären Linsenkapselveränderungen mit 10,8\% aller Augen in unserer Untersuchung vergleichsweise gering. Lundvall und Kollegen berichten noch von einem sehr hohen Anteil von Patienten mit VAO (70\%) (Lundvall und Zetterström 2006). Der Anteil bei Trivedi et al. (Trivedi et al. 2011) lag bei 37,9\% der Patienten. Die Untersuchung von Lu und Kollegen zeigt einen Anteil von 26,9\% (Lu et al. 2010). Die Autoren erklären diesen geringeren Prozentsatz durch das Lebensalter ihrer Patienten (bei Kataraktextraktion älter sechs Monaten) und dementsprechend einem verringerten Risiko von sekundären Linsenkapselveränderungen (Hosal und Biglan 2002). Bei einem durchschnittlichen Alter der Kataraktextraktion von 19 Wochen ist diese Erklärung bezüglich unserer Ergebnisse unzureichend. Die guten Ergebnisse unserer Patienten könnten darauf basieren, dass der Anteil der primär implantierten Augen an der Gesamtsumme relativ gering ist. In dieser Gruppe ist jedoch der Anteil von sekundären Linsenkapselveränderungen mit 60\% der Augen sehr hoch. Außerdem könnten die Ursachen in der exakten Durchführung von Operationstechniken liegen. So wurden zwar in den zitierten Untersuchungen wie auch in unserer Untersuchung eine Kapsulorhexis und eine vordere Vitrektomie durchgeführt, jedoch ist die Größe der Kapsulorhexis zwischen Operateuren unterschiedlich.

Auf die gesamte Studienpopulation und den Untersuchungszeitraum von fünf Jahren gesehen lag der Anteil der VAO in der IATS bei 41\% der Augen. Als interessant zeigt sich, dass mit 68\% vs. $14 \%$ der Anteil in der primären IOL-Gruppe vergleichsweise hoch ist (Plager et al. 2014). Ähnliches bildet sich in unserer Untersuchung ab. Hier liegt der Anteil in der primär implantierten Gruppe mit 60\% weitaus höher als jener in der sekundär implantierten Gruppe mit 6,2\% und dem der aphaken Gruppe mit 5,9\%. Eine mögliche Erklärung wäre, dass die IOL in der Kapsel verhindert, dass sich vordere und hinter Kapsel zusammenlegen und es demzufolge zur Sequestrierung und Proliferation von Linsenepithel kommen kann. Im aphaken Auge bilden die Sequester in der Regel einen Soemmering'schen Nachstar, welcher jedoch länger wachsen muss, um einen Einfluss auf das Sichtfeld zu haben (Plager et al. 2011). 


\subsubsection{Glaukom}

Die Entwicklung eines Glaukoms ist eine ernste Spätkomplikation im Verlauf nach Lentektomie bei Kindern (Simon et al. 1991). Vorangegangene Untersuchungen zeigen dabei einen signifikanten Anteil an Patienten mit Glaukom-Entwicklung (Egbert et al. 1995; Kim et al. 2012; Lambert et al. 2013; Freedman et al. 2015; Solebo et al. 2015). Eine aktuelle Metaanalyse berichtet über das Auftreten des Glaukoms in 17\% der Fälle bei mittlerem Alter der Kataraktextraktion von drei Monaten und mittlerem Glaukom-Auftreten bei 4,3 Jahren (Mataftsi et al. 2014). Dies deckt sich nicht mit unserer Untersuchung, bei der es nur bei einem Patienten an einem Auge $(1,4 \%)$ zu sechs Eingriffen kam.

Die Schwierigkeit im Vergleich der bestehenden Literatur besteht im Mangel einer einheitlichen Glaukom-Definition beziehungsweise einheitlicher diagnostischer Kriterien oder auch an der unterschiedlichen Nachkontrolle aufgrund der retrospektiven Auswertung. Der kleine Anteil des Glaukoms am Gesamtkollektiv der Augen in unserer Untersuchung erklärt sich unter anderem durch die Einschränkung auf operationsbedürftige Glaukome, da, wenn möglich, eine konservative Therapie vorgezogen wurde. Freedman und Kollegen untersuchten in der IATS zur besseren Übersichtlichkeit Glaukom-Patienten und Glaukom-suspekte Patienten sowie zusätzlich Glaukom-bedinge Operationen. Zum Fünf-Jahres-Zeitpunkt zeigten sich insgesamt 20 Augen mit Glaukom (18\%) und 16 zusätzliche Glaukom-suspekte Augen (insgesamt 36 Augen (32\%)). Dabei fand sich kein signifikanter Unterschied zwischen den Behandlungsgruppen. Zu Operationen kam es jedoch nur in acht Fällen bei sieben Augen (6,1\%) (Freedman et al. 2015). In vielen der Glaukom-Fälle konnte der intraokulare Druck demnach durch eine konservative Therapie kontrolliert werden. Bezüglich unserer Population liegt daher die Vermutung nahe, dass die meisten der Glaukom-Patienten schon durch konservative Maßnahmen behandelt werden konnten.

Während Mataftsi und Kollegen in ihrer Metanalyse noch einen protektiven Effekt der primären IOL-Implantation nachweisen konnten (hazard ratio 0,1; $\mathrm{p}=0,02$ ) (Mataftsi et al. 2014), konnte zum Fünf-Jahres-Zeitpunkt der IATS, welche dank prospektivem Design und Randomisierung einem weitaus geringeren Bias ausgesetzt ist, weder bezüglich der Glaukom-Patienten noch bezüglich der Glaukom- und Glaukom-suspekten Patienten ein signifikanter Unterschied zwischen den Gruppen festgestellt werden (Freedman et al. 2015). Gestützt wird die Aussage zum Mangel an protektivem Einfluss durch IOL-Implantation durch die IoLunder2-Studie (Solebo et al. 2015). Es fällt schwer, diese Aussage anhand der vorliegenden Untersuchung zu diskutieren. Zum einen ist die Gruppe der primär versorgten Patienten in unserer Untersuchung sehr klein ausgefallen, zum anderen wurde diese Gruppe durchschnittlich nur 3,76 Jahre nachuntersucht. Verglichen mit dem mittleren Auftreten bei Mataftsi et al. von 4,3 Jahren ließe sich spekulieren, dass die Population der primär Versorgten in dieser Untersuchung nicht lang genug verfolgt wurde, um von etwaigen operationsbedürftigen Komplikationen zu erfahren. Da die Gruppe der aphaken Patienten und die Gruppe der sekundär versorgten Patienten eine ähnliche Größe und einen ähnlich langen Nachuntersuchungszeitraum aufweisen, könnte spekuliert werden, dass sich die Linsenimplantation auf das Glaukom-Risiko auswirkt. Jedoch findet sich in der Literatur nirgends 
ein Hinweis auf eine solche Korrelation. Eher wahrscheinlich ist, dass der betroffene Patient auch andere Risikofaktoren aufwies, die nachgewiesenermaßen die Glaukom-Entstehung fördern, wie zum Beispiel ein junges Lebensalter bei Kataraktextraktion (Trivedi et al. 2006; Chak und Rahi 2008; Freedman et al. 2015) oder ein Mikrophthalmus (Asrani et al. 2000; Donahue et al. 2000; Chak und Rahi 2008; Solebo et al. 2015). Möglicherweise könnte auch der postoperative Entzündungsreiz eine Rolle in der Glaukom-Entstehung spielen. Eine engmaschige lebenslange Kontrolle der Patienten scheint daher indiziert. Insbesondere da es, ähnlich den Ergebnissen unserer Untersuchung, häufig zu multiplen chirurgischen Interventionen am selben Auge kommen kann (Papadopoulos und Khaw 2003; Papadopoulos et al. 2007).

\subsubsection{Synechien}

Die Synechienbildung stellt mit 20\% aller komplikationsbehafteten Augen die dritthäufigste Komplikation in unserer Untersuchung dar. Betrachtet man den Anteil an den Komplikationen zeigt sich, dass linsenversorgte Patienten häufiger Synechien-bedingt reoperiert werden mussten als die Patienten der aphaken Gruppe (primär 16,7\% vs. sekundär 16\% vs. aphak 0\%). Dieser Umstand lässt vermuten, dass die Synechien eine kunstlinsenbezogene Komplikation darstellen. Der Unterschied könnte demnach entweder im Design und Material der Linse, in zusätzlichen operativen Traumata, im Rahmen der Linsenimplantation oder in den veränderten Platzverhältnissen im Auge nach der Linsenimplantation liegen. Bezogen auf die Zahl der Patienten in den Gruppen sind primär versorgte Patienten zu 25\% und sekundär versorgte Patienten zu 8,3\% von Synechien betroffen. Dieser Unterschied könnte durch die geringe Anzahl der primär versorgten Patienten befördert sein. Daneben könnte vermutet werden, dass die entwickelten Augen bzw. Kinder eine höhere Resilienz gegenüber dem Fremdkörper Linse oder dem Eingriff haben und es demzufolge zu weniger entzündlichen Reaktionen kommt. Kritisch sei gesagt, dass die Auswahl der Patienten zur primären Linsenversorgung selektiv stattfand. Es ist also gut möglich, dass eine unzureichende Compliance bezüglich der Kontaktlinsenversorgung durch die Eltern zur Entscheidung der primären Implantation führte und dementsprechend ebenso keine ausreichende Compliance bezüglich der postoperativen Versorgung und Hygiene bestand.

Auch in diesem Fall ergeben sich Schwierigkeiten im Literaturvergleich durch verschiedene Begrifflichkeiten. Im Folgenden wird angenommen, dass Ausdrücke wie corectopia und pupile capture äquivalent zum Begriff Synechie sind. Die IATS zeigt eine ähnliche Verteilung bezüglich der aphaken und der primär-versorgten Gruppe. Plager et al. beschreiben einen Anteil von Patienten mit corectopia in $4 \%$ der aphaken und $28 \%$ der primär versorgten Gruppe zum Fünf-JahresZeitpunkt. Der Großteil wurde jedoch schon zum Ein-Jahres-Zeitpunkt beschrieben (aphak 2\% vs. primär 22,8\%) (Plager et al. 2014). Die Autoren gehen in ihrer Diskussion leider nicht weiter auf diesen Umstand ein. Lu und Kollegen vermerken pupile capture in 7,7\% ihrer primär versorgten Patienten (Lu et al. 2010) und Solebo et al. berichten über corectopia in insgesamt 3\% der Augen (Solebo et al. 2015). Leider lässt sich nicht nachvollziehen, welcher Anteil der genannten Patienten operationsbedürftig war. Trotzdem scheinen sich unsere Ergebnisse mit den beschriebenen annähernd zu decken. 


\subsubsection{Unilateral vs. bilateral}

In Hinsicht auf die Lateralität ist unsere Population bezüglich der Augen sowohl im Sinne des Versorgungstyps (siehe Tabelle 5, insgesamt $54,1 \%$ vs. 45,9\%) als auch des Nachuntersuchungszeitraums (7,97 Lj. vs. 7,23 Lj.) gleichmäßig verteilt. Unsere Untersuchung zeigt, dass Augen von Kindern mit bilateraler Katarakt signifikant häufiger $(p=0,0386)$ Komplikationen aufweisen (Sekundäre Linsenkapselveränderung $20 \%$ vs. 5,9\%, IOLLuxation/Subluxation $20 \%$ vs. 5,9\%, Synechien $10 \%$ vs. 5,9\%). Betrachtet man den Implantationszeitpunkt, erkennt man, dass bilateral betroffene Kinder früher operiert wurden als unilateral betroffene Patienten. Das liegt daran, dass unilaterale kongenitale Linsentrübungen erst später auffallen (s. o.). Ein früher Operationszeitpunkt bietet sich demnach als mögliche Erklärung für das gehäufte Auftreten von Komplikationen bei Patienten mit bilateraler Katarakt an und wurde schon von anderen Autoren vermutet (Lundvall und Kugelberg 2002a). Solebo und Kollegen konnten zum Beispiel zeigen, dass für bilateral betroffene Kinder ein früher Extraktionszeitpunkt der einzige unabhängige Prädiktor bezüglich des Auftretens von Glaukomen ist (Solebo et al. 2015). Eine hohe Komplikationsrate verschlechtert dabei das Ergebnis der Behandlung (Chak et al. 2006; Zhang et al. 2016).

Insbesondere bei der bilateralen Katarakt steht dem jedoch entgegen, dass ein jüngeres Alter zum Zeitpunkt der Kataraktextraktion auch positiv mit dem visuellen Ergebnis korreliert (Lundvall und Kugelberg 2002b; Chak et al. 2006; Magli et al. 2013; Solebo et al. 2015).

Da Patienten mit bilateraler Katarakt, anders als die mit unilateraler Katarakt, vollkommen auf den Erfolg der Behandlung angewiesen sind, empfiehlt sich aus den Ergebnissen unserer Daten, dass Behandler in Anbetracht des erhöhten Auftretens von Komplikationen, beeinflussbare Faktoren wie die frühe Operation, die strikte Kontrolle von Komplikationen und eine hohe Compliance bei der Amblyopieprophylaxe (Rong et al. 2015; Zhang et al. 2016) besonderes beobachtet werden sollte, um das Risiko einer Sehverschlechterung zu minimieren.

\subsection{Bulbuslänge}

Die Bulbuslänge bzw. deren Entwicklung ist nicht nur ein wichtiger Parameter zur Berechnung der passenden IOL. Kim und Kollegen verweisen in ihrer Multivariatanalyse darauf, dass eine längere Achse im Zusammenhang mit einem schlechteren Visusergebnis steht (Kim et al. 2012).

\subsubsection{Versorgungstyp}

Der Vergleich der Bulbuslänge zwischen den Versorgungstypen in unserer Untersuchung zeigt, dass bis zum Alter von zwei Jahren die durchschnittliche Bulbuslänge der drei Gruppen keine großen Unterschiede aufweisen. Das überrascht in Anbetracht des hohen Anteils an Patienten mit Mikrophthalmus in der Gruppe der primär versorgten Patienten (20\%). Trivedi und Wilson ermittelten in ihrer Untersuchung des Einflusses der primären IOL-Implantation auf das interokulare Wachstum, dass präoperativ kurze Augen postoperativ mehr und präoperativ lange 
Augen weniger wuchsen (Trivedi und Wilson 2007b). Ein solcher Einfluss könnte erklären, warum die primär versorgten Augen im Vergleich zu den aphak belassenen Augen der anderen beiden Gruppen im Durchschnitt eine ähnliche Länge erreichen.

In der deskriptiven Betrachtung entsteht im Altersbereich von drei bis sechs Lebensjahren der Eindruck eines längeren Durchmessers der primär versorgten Augen. Dem widerspricht die statistische Testung, bei der in keinem der Zeiträume ein signifikanter Unterschied festgestellt werden kann. Dieser Widerspruch könnte jedoch durch die geringe Augenzahl (drei Augen) der primären Gruppe in diesem Vergleich befördert sein. Während Griener und Kollegen (Griener et al. 1999) noch von einem verminderten Längenwachstum von Augen mit primärer IOLVersorgung sprachen, kamen die Autoren der IATS zu einem anderen Ergebnis. In ihrer Untersuchung gab es zur Baseline-Untersuchung keinen Unterschied zwischen den aphaken und den primär implantierten Augen. Der zum Ein-Jahres-Zeitpunkt festgestellte Unterschied (IOLGruppe um 0,6 mm länger, $p=0,009$ ) (Lambert et al. 2012) übertrug sich dabei jedoch nicht auf die weitere Untersuchung. Zum Fünf-Jahres-Zeitpunkt zeigte sich bezüglich der Längenänderung kein signifikanter Unterschied (3,4 mm IOL vs. 3,2 mm CL, p=0,53) (Wilson et al. 2017). Dieses Langzeitergebnis deckt sich mit den Ergebnissen unserer Untersuchung.

\subsubsection{Unilateral vs. bilateral}

Vergleicht man einseitig oder beidseitig betroffene Kinder, erscheinen Augen von Kindern mit unilateraler Katarakt in unserem Vergleich länger als Augen der Kinder mit bilateraler Katarakt. Diese Beobachtung zieht sich durch alle Altersklassen. Es deckt sich mit Berichten von Cappozzi und Kollegen und Lin und Kollegen. Erstere berichten, dass Augen mit unilateraler Katarakt innerhalb der ersten 42 Lebensmonate einen signifikant längeren Bulbusdurchmesser aufwiesen als Augen mit bilateralem Katarakt ( $p=0,029)$ (Capozzi et al. 2008). Lin und Kollegen postulieren eine längere Achse der Patienten mit unilateraler Katarakt als der mit bilateraler Katarakt in allen Altersgruppen (Lin et al. 2016). Da Trivedi und Wilson bei Patienten unter 60 Monaten von einer kleineren Achslänge in unilateralen Augen und erst über 60 Monaten von einer größeren Achslänge in unilateralen Augen berichteten (Trivedi und Wilson 2007a), wurde von Lin und Kollegen vermutet, dass ethnische Zugehörigkeit (kaukasisch oder afroamerikanisch vs. chinesisch) ein Grund für diese unterschiedlichen Ergebnisse sein könnte (Lin et al. 2016). Dem widerspricht die vorliegende Untersuchung. Zwar wurde die Ethnie nicht explizit dokumentiert, jedoch rekrutieren sich die Patienten aus dem Göttinger Umland, das durchaus in der Mehrheit als kaukasisch bezeichnet werden kann. Näher liegt es also, etwaige Ursachen im unterschiedlichen Studientyp, in der Art der Gruppierung oder der Größe der Population zu suchen. Die wahrscheinlichste Erklärung ist, dass eine unilaterale kongenitale Katarakt zu schlechterem visuellen Ergebnis führt, das wiederum das Augenlängenwachstum stimuliert. 


\subsubsection{Geschlecht}

In unserem Datensatz haben männliche Patienten einen größeren Bulbusdurchmesser als weibliche Patienten. Signifikante Unterschiede sind in unserer Untersuchung jedoch nur im Zeitraum bis zum zweiten Lebensjahr nachzuweisen $(\mathrm{p}=0,0257)$. Lin und Kollegen kommen im Altersbereich von zwei bis sechs Jahren zum gleichen Ergebnis (Lin et al. 2016), während Capozzi et al. keinen Unterschied zwischen den Geschlechtern feststellen konnten (Capozzi et al. 2008). Trivedi und Wilson hingegen zeigten, entsprechend unseren Beobachtungen, einen kürzeren durchschnittlichen Bulbusdurchmesser in Mädchen sowohl in Augen mit Katarakt als auch in gesunden Augen (Trivedi und Wilson 2007a). Lin et al. vermuten, dass die Differenz im besagten Zeitraum aufgrund der Korrelation zwischen Kopfumfang und Bulbuslänge und dementsprechend wegen des größeren Kopfumfanges von Jungen gegenüber Mädchen im Alter von fünf Jahren besteht (Lin et al. 2016). Dass sich die Differenz in unserer Untersuchung durchgängig vermuten lässt obwohl sie nur im frühen Lebensalter nachweisbar ist, könnte möglicherweise an der abnehmenden Zahl der Augen liegen. So zeigt der paarweise t-Test in der weniger starken Gruppe zweites bis sechstes Lebensjahr noch einen Wert von $\mathrm{p}=0,0778$ und in der schwach besetzten Gruppe siebtes Lebensjahr und älter einen Wert von $p=0,174$. Da eine Differenz zwischen den Geschlechtern möglicherweise Auswirkungen auf die Kalkulation der Linsenstärke hätte wäre eine prospektive Untersuchung mit geringerem Bias und einer größeren Population jedoch vor einem solchen Schluss angebracht. Sehr wahrscheinlich handelt es sich um einen Effekt der Gruppengröße und nicht um ein tatsächlich geschlechtsgebundenes Phänomen.

\subsubsection{Patienten mit sekundärer Linsenkapselveränderung}

Die getrennte Untersuchung aller Patienten mit der Komplikation sekundäre Linsenkapselveränderung zeigt einen Unterschied in der deskriptiven Betrachtung. Im Zeitraum null bis zwei Lebensjahre sind die Mittelwerte jedoch nur an der Grenze zu signifikant kleiner $(p=0,0527)$ als jene der Patienten ohne sekundäre Linsenkapselveränderung. Daneben lassen sich keine signifikanten Unterschiede ermitteln. Dies könnte dabei, gerade im Abschnitt von null bis zwei Lebensjahre, an gehäuften und früheren Messungen bei Kindern mit sekundärer Linsenkapselveränderung im Gegensatz zu ihren nicht von sekundärer Linsenkapselveränderung betroffenen Pendants liegen. Wegen des großen Wachstums im ersten Lebensabschnitt hat der Messzeitpunkt eine größere Auswirkung auf den Durchschnitt der Bulbuslänge als in den späteren Zeiträumen. Diese Vermutung wird sowohl durch das gehäufte Auftreten von Kataraktoperationen im ersten Lebensjahr gestärkt (Plager et al. 2014) als auch durch den Einfluss des Lebensalter auf die Achslänge, insbesondere bei Kindern unter einem Lebensjahr (Capozzi et al. 2008).

\subsubsection{Rate of Axial Growth - RAG}

Um den Vergleich zwischen Patienten mit unterschiedlichem Operationszeitpunkt und unterschiedlichen Nachuntersuchungszeiträumen zu verbessern, entwickelten Vasavada et al. den 
Begriff der rate of axial growth (Vasavada et al. 2004). Nach Anwendung der Ausschlusskriterien einer Baseline-Messung eine Woche um die Kataraktextraktion und eines MindestNachuntersuchungszeitraums von mehr als zwei Jahren verblieben uns 33 Augen zur Untersuchung. Leider konnte die Gruppe der primär versorgten Patienten nur mit einem Auge zur Untersuchung beitragen, was einen Vergleich zwischen den Versorgungstypen unmöglich macht.

Zum Zeitpunkt der Baseline-Untersuchung kommt unser Vergleich zu einem signifikanten Unterschied bezüglich der Lateralität (bilateral 16,91 +/- 0,37 mm vs. unilateral 18,58 +/$0,42 \mathrm{~mm}, \mathrm{p}=0,0064)$ und der Patienten mit und ohne sekundäre Linsenkapselveränderung (mit 16,26 +/- 0,09 mm vs. ohne 17,82 +/- 0,32 mm, $\mathrm{p}<0,0001)$. Dies deckt sich mit den im Verlauf des Kapitels 4.2 genannten Ergebnissen.

Zum Zeitpunkt der letzten Messung besteht nur noch ein Unterschied bezüglich der Lateralität (bilateral 20,51 +/- 0,58 mm vs. unilateral 22,27 +/- 0,6 mm, p=0,0457). Dass sich zu diesem Zeitpunkt kein Unterschied mehr zwischen den Patienten mit sekundärer Linsenkapselveränderung und ihren nicht von sekundärer Linsenkapselveränderung betroffenen Pendants findet, stützt die in Kapitel 4.2.4 genannte These des Einflusses des Lebensalters.

Im absoluten Wachstum konnten in unserer Untersuchung keine Unterschiede bezüglich der untersuchten Parameter mehr festgestellt werden. Sminia und Kollegen fanden hier nur einen grenzwertig signifikanten Unterschied $(\mathrm{p}=0,049)$ zwischen operierten und nicht operierten Augen (Sminia et al. 2010). Die Autoren der IATS konnten diesbezüglich keinen Unterschied zum FünfJahres-Zeitpunkt ermitteln (Wilson et al. 2017) ähnlich wie Tadros und Kollegen (Tadros et al. 2016). Anders als diese Untersuchung postuliert die IATS jedoch einen Unterschied im absoluten Wachstum bezüglich der Patienten mit operativ bereinigter visueller Achse. Der Unterschied im Wachstum zwischen komplikationsbehafteten Patienten und gesunden Patienten betrug dabei zum Fünf-Jahres-Zeitpunkt im Durchschnitt $1,1 \mathrm{~mm}(\mathrm{p}=0,013)$ (Wilson et al. 2017). Der durch die Autoren angestellten Vermutung, dass eine Verlegung der visuellen Achse zum exzessiven Bulbuswachstum führt, kann in Anbetracht der kleinen Population und des Studientyps unserer Untersuchung leider nur sehr begrenzt widersprochen werden.

Bei der Betrachtung der RAG konnte unsere Untersuchung keinerlei signifikanten Einfluss geltend machen. Zu den gleichen Ergebnissen kamen auch Sminia und Kollegen (Sminia et al. 2010). Im Vergleich von Patienten mit unilateraler Katarakt und bilateraler Katarakt erwähnen Vasavada et al. einen Unterschied bezüglich der Lateralität (unilateral 25,53\% vs. bilateral 18,5\%, p=0,001) (Vasavada et al. 2004). Während sich diese Aussage mit den Beobachtungen aus Kapitel 4.2.2 deckt, bietet sich in der Untersuchung der RAG keine Entsprechung. Dies mag an der vergleichsweise kleinen Menge der an der Untersuchung beteiligten Augen liegen.

Ähnlich Vasavada und Kollegen (Vasavada et al. 2004) konnte auch die vorliegende Untersuchung keinen Einfluss von VAO auf die RAG darstellen. 


\subsection{Sphärisches Äquivalent}

Das sphärische Äquivalent zum Zeitpunkt der letzten Messung dient in unserer Untersuchung in der Hauptsache der Kontrolle des Erfolges der IOL-Implantation bzw. deren Berechnung. Die Linsen der Patienten in unserer Untersuchung wurden nach den gängigen Sanders-Retzlaff-Kraff (SRK)/theroretical-(T)-, Holladay-I- und SRK-II-Formeln ausgewählt.

Die deskriptive Analyse zeigt in der Altersgruppe von sieben Lebensjahren und älter einen ausgeprägten Unterschied zwischen den Versorgungstypen. Dabei ist insbesondere auf den Unterschied zwischen primär und sekundär versorgten Patienten zu verweisen (siehe Abbildung 3). Diese Beobachtung bestätigt sich sowohl im paarweisen t-Test $(p<0,0001)$ als auch in der Variatanalyse, welche den Einfluss des Versorgungstyps auf das finale sphärische Äquivalent bestätigt. Kritisch ist sowohl die kleine Anzahl an primär versorgen Augen in der Untersuchung als auch das Einbeziehen der aphaken Patienten zu sehen. So ist nicht zu unterscheiden, ob sich die nötige Stärke des Einflusses auf die Varianzanalyse zum Großteil aus der Gruppe der aphaken Patienten rekrutiert, die natürlich zum Zeitpunkt der letzten Messung ohne Kontaktlinsenversorgung noch immer stark hyperop sind, oder aus dem großen Abweichen der geringen Anzahl primär-versorgter Patienten. Magli und Kollegen berichten, ähnlich dieser Untersuchung, einen signifikanten Unterschied des sphärischen Äquivalents zwischen den primär und sekundär implantierten Patienten zur letzten Untersuchung (primär -2,5 +/- 3dpt vs. sekundär 0,4 +/2,3dpt, $\mathrm{p}=0,02$ ) (Magli et al. 2013). Weakley und Kollegen berichten im Zuge der IATS zum FünfJahres-Zeitpunkt einen durchschnittlich niedrigeren „Myoptic Shift“ (Differenz zwischen dem sphärischen Äquivalent zur ersten und zur letzten Messung) in CL Patienten als in Patienten mit primärer IOL. Die Autoren schlagen daher vor, die Stärke der primären IOL um 2,5 dpt anzupassen. Kritisch geben sie zu bedenken, dass sich nur etwa $25 \%$ der Patienten innerhalb von einer dpt um den erwarteten Zielwert befanden. 50\% der Augen lagen innerhalb von drei dpt und die anderen 50\% zwischen drei und 14 dpt um den errechneten Zielwert (Weakley et al. 2017). Diese große Variabilität könnte erklären, warum die Differenz gerade in Anbetracht der kleinen Augenzahl der primär versorgten Gruppe in unserer Untersuchung so groß ist.

Des Weiteren konnten wir in der Varianzanalyse das Alter zur Refraktionsmessung als Einflussfaktor ausmachen. Dies erklärt sich wahrscheinlich aus der größeren Gruppe der sekundär versorgten Patienten, die bei frühem Operationszeitpunkt leicht überkorrigiert wurden.

\subsubsection{Unilateral vs. bilateral}

Rong und Kollegen berichten in bilateralen Fällen einen Refraktionsfehler von 3,11dpt +/- 2,45dpt und in unilateralen Fällen Fehler von -2,39dpt +/- 2,77dpt (Rong et al. 2015). Dieser entsprachen im Allgemeinen den vorhergegangenen Untersuchungen (Kim et al. 2012; Magli et al. 2013). Rong et al. konnten des Weiteren neben anderen Faktoren eine Assoziation zwischen dem Refraktionsfehler und dem finalen Visus herstellen (unilateral $p<0,001$ vs. bilateral $p=0,043$ ) (Rong et al. 2015). Demgegenüber lässt die deskriptive Analyse unserer Untersuchung bezüglich der Lateralität im Zeitraum von sieben Lebensjahren und älter die Vermutung zu, dass unilaterale 
Patienten einen größeren Refraktionsfehler aufweisen als bilaterale Patienten. Dieser Einfluss bestätigt sich jedoch nicht aus der Varianzanalyse. Der Grund für den Unterschied zu den vorhergegangenen Untersuchungen ist also eher in der Studienpopulation und im Studiendesign zu suchen. Auch hier kann das Alter zur Messung des Refraktionsfehlers nachgewiesenermaßen eine größere Rolle spielen als die Lateralität.

\subsection{Problemanalyse}

Die vorliegende Untersuchung entspricht ihrer Natur als rein retrospektive Beobachtung nicht den höchstmöglichen Anforderungen an eine klinische Studie.

Durch das Fehlen von Randomisierung sind Strukturungleichheiten zwischen den untersuchten Gruppen sehr wahrscheinlich. Eine randomisierte Erhebung könnte jedoch aus ethischen Gesichtspunkten grenzwertig sein.

Die Unkenntnis über etwaige Zielkriterien zum Zeitpunkt der Befunderhebung führt zu uneinheitlichen und nicht standardisierten Messmethoden. Dies wird durch die Verteilung auf verschiedene Mitarbeiter verstärkt.

Die Entscheidung, ob eine Komplikation operationsbedürftig ist oder nicht, war trotz klarer Kriterien subjektiv von der Einschätzung des jeweiligen Operateurs und dem Bild abhängig, dass der jeweilige Patient bot . Dies galt ebenso für die Wahl der Implantationsvariante. Ähnlich verhielt es sich mit den Ergebnissen der operativen Versorgung in deren Auswertung das Fehlen eines studienspezifischen Protokolls und der Einsatz unterschiedlicher Operateure bedacht werden muss.

Kritisch betrachtet werden sollte des Weiteren, dass zum Zeitpunkt der Dokumentation eine weitere wissenschaftliche Bearbeitung nicht antizipiert werden konnte und daher nötige, unsere Untersuchung betreffende Informationen fehlten oder nicht in ausreichender Form dokumentiert waren. Außerdem ist zu beachten, dass ein kleiner Teil der benötigten Patientenakten trotz ausgiebiger Versuche nicht auffindbar war und daher nicht in die Untersuchung mit einbezogen werden konnte.

Ein weiteres Problem stellt die geringe Fallzahl dar. Dies wird insbesondere bei der Betrachtung der einzelnen Subgruppen relevant. Um größere Kollektive von Patienten einer seltenen Erkrankung zu vergleichen, wäre jedoch eine multizentrische Studie erforderlich. Eine solche würde auch ermöglichen, den Einfluss von Regionalität, wie in unserer Arbeit vorhanden, auf die Untersuchungsgruppe zu reduzieren.

Unterschiede im Nachuntersuchungszeitraum sind im Charakter der Studie unvermeidlich. Dies macht insbesondere den Vergleich primär implantierter Patienten schwierig. In Bezug auf dieses Patientenkollektiv ist außerdem unklar, aus welchen Gründen sich die Operateure für eine primäre IOL-Implantation entschieden haben. Dementsprechend können die Ursachen etwaiger Unterschiede schon im Patientengut, unabhängig von der Implantationsmethode, liegen. 


\subsection{Ausblick und Perspektive}

Die Diskussion um die optimale Versorgung von Patienten mit kindlicher Katarakt ist weiterhin aktuell. Die Positionen reichen von der Empfehlungen zur primären Implantation bei Kindern unter zwei Jahren (Sukhija et al. 2014) über den Vorschlag zur differenzierten Betrachtung (Plager et al. 2014) bis hin zur Empfehlung zur sekundären IOL- Implantation nach zwei Jahren (Solebo et al. 2015). Zwar laufen mit der IATS und der IoLunder2-Studie zwei vom Studiendesign gesehen qualitativ hochwertige Studien, jedoch fehlt bisher die langfristige Analyse gerade bezüglich der Patienten mit sekundärer IOL-Implantation. Die vorliegende Untersuchung bietet weiteren Diskussionsstoff, vor allem in Anbetracht der Tatsache, dass ein Großteil der Komplikationen der sekundär implantierten Patienten die seltene Komplikation der IOL-Luxation/-Subluxation darstellt. Sollte sich dieses Auftreten in anderen Untersuchungen nicht bestätigen, kann aus den vorliegenden Daten der Hinweis gewonnen werden, dass entsprechend der IoLunder2-Studie, die sekundäre IOL-Implantation das komplikationsärmere Verfahren bei gleichem funktionellen Ergebnis darstellt. Es bleibt also abzuwarten, zu welchen Ergebnissen langfristig angelegte und bestenfalls prospektive, randomisierte Studien gelangen.

Neuere Entwicklungen, wie zum Beispiel die bag-in-lens, oder das optic capture, welche vielversprechende Ergebnisse bezüglich der Entwicklung von sekundären Linsenkapselveränderungen aufzeigen, würden in Anbetracht der Tatsache, dass das Risiko für sekundäre Linsenkapselveränderungen in der Gruppe mit primärer IOL-Implantation weitaus höher war, eine erneute Evaluation der Risiken nötig machen.

Zum Gesamtbild gehören in der Abschätzung der individuellen patientengerechten Versorgung jedoch neben den Komplikationen auch funktionelle Ergebnisse wie der Visus und der Refraktionsfehler. Breite Schwankungen im Erreichen der Zielrefraktion bei primär implantierten Patienten (Weakley et al. 2017) machen möglichweise das Tragen von Korrekturen nötig, welche sich bei sekundärer Versorgung vermeiden ließen.

Bei weiteren multiplen Einflussfaktoren auf das Ergebnis, wie dem Alter zur Kataraktextraktion, dem Katarakttyp, der Compliance in der Amblyopietherapie oder der Lateralität (Rong et al. 2015), welche sich in manchen Fällen in ihrem Einfluss entgegenstehen, bleibt dem Behandler weiterhin nur die sorgfältige, patientenspezifische Abwägung aller Faktoren, um ein optimales Langzeitergebnis zu erreichen. 


\section{Zusammenfassung}

Hintergrund: Ziel der vorliegenden Arbeit war es, das Spektrum und die Häufigkeit der intra- und postoperativen Komplikationen bei Kindern mit behandlungsbedürftiger kongenitaler Linsentrübung zu erfassen. Daneben sollte die Entwicklung der Bulbuslänge und des Refraktionsfehlers der Patienten der Augenklinik der Universitätsmedizin Göttingen im Zeitraum von 1990 bis 2015 untersucht werden.

Material und Methoden: Es handelt sich um eine retrospektive Analyse. Alle Patienten wurden im ersten Lebensjahr an der Augenklinik der Universitätsmedizin Göttingen operiert. Die auftretenden Komplikationen und daraus resultierenden Operationen wurden kategorisiert und innerhalb der Gruppen bezüglich der Art der Linsenversorgung sowie der Lateralität verglichen. Die Bulbuslänge wurde im Verlauf dokumentiert, nach Alter gruppiert und auf Einfluss des Versorgungstyps, der Seitigkeit, des Geschlechts und des Auftretens von sekundären Linsenkapselveränderungen untersucht. Eine gesonderte Untersuchung bezüglich des Einflusses einiger dieser Parameter auf die rate of axial growth (RAG) wurde anhand von Patienten mit Messwerten +/- eine Woche um die Kataraktextraktion und einem Nachuntersuchungszeitraum von mindestens zwei Jahren durchgeführt. Der Refraktionsfehler wurde zum letzten Kontakt als sphärisches Äquivalent erhoben und bezüglich des Einflusses von Lebensalter, Versorgungstyp, Ein- oder Beidseitigkeit und Komplikationen betrachtet. Die statistische Auswertung erfolgte mittels absoluter und relativer Häufigkeit, Mittelwert und Standardabweichung, t-Test und Varianzanalyse.

Ergebnisse: Die Untersuchung umfasst 54 Patienten (30 männlich und 24 weiblich) mit 74 Augen. Darunter fanden sich 28,4\% aphake, 6,7\% primär implantierte und 64,9\% sekundär versorgte Augen bei 54,1\% bilateraler und 45,9\% unilateraler Katarakt. Das Alter zur Kataraktextraktion war im Mittel bei 134,7 Tagen und der mittlere Nachuntersuchungszeitraum betrug 7,5 Jahre.

Primär implantierte Patienten hatten mehr Komplikationen als aphak belassene Patienten (FisherExakt-Test $\mathrm{p}=0$,0335). Zudem hatten Patienten mit bilateraler Katarakt häufiger Komplikationen als Patienten mit unilateraler Katarakt (Fisher-Exakt-Test p=0,0386), was aber nicht an der höheren Eingriffszahl der beidseitig betroffenen Kinder lag. Die häufigsten Komplikationen waren die sekundäre Linsenkapselveränderung in 10,8\% der Augen, die IOL-Luxation/-Subluxation in 10,8\% der Augen, die überraschenderweise nur die Gruppe der sekundär implantierten Augen betraf, und Synechien (6,7\%). Der Anteil der Patienten mit sekundärer Linsenkapselveränderung war in der Gruppe der primär implantierten Patienten am höchsten (60\%) während Synechien nur primär implantierte Patienten (25\%) und sekundär implantierte Patienten $(8,3 \%)$ betrafen.

Der Achsendurchmesser ist in der Analyse über alle Zeiträume bei Patienten mit bilateraler Katarakt signifikant geringer als bei Patienten mit unilateraler Katarakt (Geburt - zweites Lj. $\mathrm{p}=0,0004$, drittes bis sechstes Lj. $\mathrm{p}=0,0051$, siebtes $\mathrm{Lj}$. und älter $\mathrm{p}=0,0077$ ) und bei männlichen Patienten im Zeitraum von Geburt bis ins zweite Lebensjahr signifikant länger als bei weiblichen 
$(\mathrm{p}=0,0257)$. Im gesonderten Vergleich der RAG kann kein Unterschied zwischen den Gruppen festgestellt werden.

Die Varianzanalyse der Einflussfaktoren für den finalen Refraktionsfehler zeigt den Versorgungstyp $(p=0,0188)$ und das Alter zur Refraktionsmessung $(p=0,0016)$ als einzig signifikante Parameter. Ein Vergleich der Versorgungstypen, betreffend den finalen Refraktionsfehler, zeigt im paarweisen t-Test bezüglich des Zeitraums von sieben Lebensjahren und älter jeweils einen signifikanten Unterschied $(p<0,0001)$. Dabei hatten sekundär mit Kunstlinse versorgte Kinder einen geringeren Brechkraftfehler als primär mit Kunstlinse versorgte Kinder.

Schlussfolgerung: Unsere Untersuchung zeigt, dass aphak belassene Patienten signifikant seltener von Komplikationen betroffen sind als primär implantierte Patienten. Nimmt man den finalen Refraktionsfehler als Maßstab des Erfolges der adäquaten Linsenimplantation, zeigt sich, dass mit primärer Kunstlinse versorgte Patienten eine größere Abweichung vom Zielwert zeigen und dementsprechend die Versorgung mit Kontaktlinsen oder die sekundäre IOL-Implantation empfohlen werden sollte. 


\section{$6 \quad$ Literaturverzeichnis}

Ahmadieh H, Javadi MA, Ahmady M, Karimian F, Einollahi B, Zare M, Dehghan MH, Mashyekhi A, Valaei N, Soheilian M, Sajjadi H (1999): Primary capsulectomy, anterior vitrectomy, lensectomy, and posterior chamber lens implantation in children: limbal versus pars plana1. J Cataract Refract Surg $\underline{25}, 768-775$

Amaya L, Taylor D, Russell-Eggitt I, Nischal KK, Lengyel D (2003): The morphology and natural history of childhood cataracts. Surv Ophthalmol $\underline{48}, 125-144$

Asadi R, Kheirkhah A (2008): Long-term Results of Scleral Fixation of Posterior Chamber Intraocular Lenses in Children. Ophthalmology $\underline{115}$, 67-72.e1

Asrani S, Freedman S, Hasselblad V, Buckley EG, Egbert J, Dahan E, Gimbel H, Johnson D, McClatchey S, Parks M, et al. (2000): Does primary intraocular lens implantation prevent ,aphakic" glaucoma in children? J AAPOS $\underline{4}, 33-39$

Bardelli AM, Lasorella G, Vanni M (1989): Congenital and developmental cataracts and multimalformation syndromes. Ophthalmic Paediatr Genet 10, 293-298

Bar-Sela SM, Spierer A (2005): Astigmatism outcomes of scleral tunnel and clear corneal incisions for congenital cataract surgery. Eye 20, 1044-1048

Bayoumi NHL (2015): Surgical Management of Glaucoma After Congenital Cataract Surgery. J Pediatr Ophthalmol Strabismus $\underline{52}, 213-220$

Birch EE, Stager DR (1996): The critical period for surgical treatment of dense congenital unilateral cataract. Invest Ophthalmol Vis Sci $\underline{37}, 1532-1538$

Birch EE, Stager D, Leffler J, Weakley D (1998): Early treatment of congenital unilateral cataract minimizes unequal competition. Invest Ophthalmol Vis Sci $\underline{39}$, 1560-1566

Birch EE, Cheng C, Stager Jr. DR, Weakley Jr. DR, Stager Sr. DR (2009): The critical period for surgical treatment of dense congenital bilateral cataracts. J AAPOS $\underline{13}, 67-71$

Bothun ED, Cleveland J, Lynn MJ, Christiansen SP, Vanderveen DK, Neely DE, Kruger SJ, Lambert SR (2013): One-Year Strabismus Outcomes in the Infant Aphakia Treatment Study. Ophthalmology $\underline{120}, 1227-1231$

Brückner R (1965): Praktische Übungen mit dem Durchleuchtungstest zur Frühdiagnose des Strabismus. Ophthalmolgica 149, 497-503

Buckley EG (2008): Safety of transscleral-sutured intraocular lenses in children. J AAPOS 12, 431-439

Capozzi P, Morini C, Piga S, Cuttini M, Vadalá P (2008): Corneal Curvature and Axial Length Values in Children with Congenital/Infantile Cataract in the First 42 Months of Life. Invest Opthalmol Vis Sci $\underline{49}, 4774$

Casteels-Rappagliosi B: Rare diseases and medical devices in the European Union. The Problem of Herbal Medicines Legal Status. Pharmaceuticals Politics and Law, Band 2. IOS Press, Amsterdamm 1999, 69-78

Chak M, Rahi JS (2008): Incidence of and Factors Associated with Glaucoma after Surgery for Congenital Cataract. Ophthalmology 115, 1013-1018.e2 
Chak M, Wade A, Rahi JS (2006): Long-Term Visual Acuity and Its Predictors after Surgery for Congenital Cataract: Findings of the British Congenital Cataract Study. Invest Opthalmol Vis Sci $\underline{47}, 4262$

Chee KYH, Lam GC (2009): Management of congenital cataract in children younger than 1 year using a 25-gauge vitrectomy system. J Cataract Refract Surg $\underline{35}, 720-724$

Churchill A, Graw J (2011): Clinical and experimental advances in congenital and paediatric cataracts. Philos Trans R Soc B Biol Sci $\underline{366}, 1234-1249$

Cleary CA, Lanigan B, O'Keeffe M (2010): Intracameral triamcinolone acetonide after pediatric cataract surgery. J Cataract Refract Surg $\underline{36}, 1676-1681$

Crouch ER, Crouch Jr ER, Pressman SH (2002): Prospective analysis of pediatric pseudophakia: Myopic shift and postoperative outcomes. J AAPOS $\underline{6}, 277-282$

Dave VP, Pathengay A, Basu S, Gupta N, Basu S, Raval V, Das T, Sharma S, Mathai A, Narayanan R, et al. (2016): Endophthalmitis After Pars Plana Vitrectomy: Clinical Features, Risk Factors, and Management Outcomes. Asia-Pac J Ophthalmol Phila Pa $\underline{5}, 192-195$

DOG/BVA - Leitlinie Nr. 26a 2010 zu Amblyopie.

Donahue SP, Byrne D, Wilson ME, Sinha D (2000): Aphakic glaucoma in children. J AAPOS 4 4, 389-390

Dutton JJ (1990): Visual rehabilitation of aphakic children: I. Editorial. Surv Ophthalmol $\underline{34}, 365$

Egbert JE, Wright MM, Dahlhauser KF, Keithahn MA, Letson RD, Summers CG (1995): A prospective study of ocular hypertension and glaucoma after pediatric cataract surgery. Ophthalmology $\underline{102}$, 1098-1101

Elston JS, Timms C (1992): Clinical evidence for the onset of the sensitive period in infancy. Br J Ophthalmol 76, 327-328

Findl O, Drexler W, Menapace R, Heinzl H, Hitzenberger CK, Fercher AF (2001): Improved prediction of intraocular lens power using partial coherence interferometry. J Cataract Refract Surg 27, 861867

Foster A, Gilbert C, Rahi J (1997): Epidemiology of cataract in childhood: A global perspective. J Cataract Refract Surg 23, 601-604

Francis PJ, Moore AT (2004): Genetics of childhood cataract. Curr Opin Ophthalmol 15, 10-15

Freedman SF, Lynn MJ, Beck AD, Bothun ED, Orge FH, Lambert SR (2015): Glaucoma-related adverse events in the first five years after unilateral cataract removal in the Infant Aphakia Treatment Study. JAMA Ophthalmol $\underline{133}$, 907-914

Fujikawa A, Mohamed YH, Kinoshita H, Tsuiki E, Kitaoka T (2018): Spontaneous dislocation of the posterior chamber intraocular lens. Int Ophthalmol $\underline{38}, 1111-1117$

Gräf M (2007): Früherkennung von Sehstörungen bei Kindern. Dtsch Arztebl 104, 724-9

Griener ED, Dahan E, Lambert SR (1999): Effect of age at time of cataract surgery on subsequent axial length growth in infant eyes. J Cataract Refract Surg 25, 1209-1213

Gross JG, Kokame GT, Weinberg DV (2004): In-the-bag intraocular lens dislocation. Am J Ophthalmol 137, 630-635 
Hartmann EE, Lynn MJ, Lambert SR (2015): Baseline Characteristics of the Infant Aphakia Treatment Study Population: Predicting Recognition Acuity at 4.5 Years of Age. Invest Ophthalmol Vis Sci $\underline{56}, 388-395$

Hayashi K, Hirata A, Hayashi H (2007): Possible Predisposing Factors for In-the-Bag and Out-of-the-Bag Intraocular Lens Dislocation and Outcomes of Intraocular Lens Exchange Surgery. Ophthalmology 114, 969-975

Hejtmancik JF (2008): Congenital cataracts and their molecular genetics. Semin Cell Dev Biol 19, 134-149

Hosal BM, Biglan AW (2002): Risk factors for secondary membrane formation after removal of pediatric cataract. J Cataract Refract Surg 28, 302-309

Jacobi PC, Dietlein TS, Jacobi FK (2002): Scleral fixation of secondary foldable multifocal intraocular lens implants in children and young adults. Ophthalmology $\underline{109}, 2315-2324$

Joshi P, Mehta R, Ganesh S (2014): Accuracy of intraocular lens power calculation in pediatric cataracts with less than a $20 \mathrm{~mm}$ axial length of the eye. Nepal J Ophthalmol $\underline{6}, 56-64$

Kim D-H, Kim JH, Kim S-J, Yu YS (2012): Long-term results of bilateral congenital cataract treated with early cataract surgery, aphakic glasses and secondary IOL implantation. Acta Ophthalmol (Copenh) 90, 231-236

Kinori M, Fabian ID, Spierer A, Wygnanski-Jaffe T, Robbins SL, Granet DB, Zion IB (2015): Measurement of Axial Length in an Office Setting Versus Under General Anesthesia in Infants and Toddlers: A Comparative Study. J Pediatr Ophthalmol Strabismus 2, 226-230

Kohnen T, Lüchtenberg M (2007): Chirurgische Therapie der kongenitalen Katarakt. Ophthalmology 104, 566-571

Kugelberg M, Zetterström C (2002): Pediatric cataract surgery with or without anterior vitrectomy. J Cataract Refract Surg 요, 1770-1773

Kugelberg U (1992): Visual acuity following treatment of bilateral congenital cataracts. Doc Ophthalmol $\underline{82}, 211-215$

Kugelberg U, Zetterström C, Syrén-Nordqvist S (1996): Ocular axial length in children with unilateral congenital cataract. Acta Ophthalmol Scand $\underline{74}, 220-223$

Kumar P, Lambert SR (2016): Evaluating the evidence for and against the use of IOLs in Infants and Young Children. Expert Rev Med Devices 13, 381-389

Lagrèze WA (2009): Die Behandlung der Katarakt im Kindesalter. Klin Monatsblätter Für Augenheilkd $226,15-21$

Lambert SR (2004): Optotype acuity and re-operation rate after unilateral cataract surgery during the first 6 months of life with or without IOL implantation. Br J Ophthalmol $\underline{88}, 1387-1390$

Lambert SR (2016): The timing of surgery for congenital cataracts. J Am Assoc Pediatr Ophthalmol Strabismus 20, 191-192

Lambert SR, Amaya L, Taylor DF (1989a): Detection and treatment of infantile cataracts. Int Ophthalmol Clin Spring $1989 \underline{29}, 51-56$

Lambert SR, Taylor D, Kriss A, Holzel H, Heard S (1989b): Ocular Manifestations of the Congenital Varicella Syndrome. Arch Ophthalmol 107, 52-56 
Lambert SR, Lynn MJ, DuBois LG, Cotsonis GA, Hartmann EE, Wilson ME (2012): Axial Elongation following Cataract Surgery during the First Year of Life in the Infant Aphakia Treatment Study. Investig Opthalmology Vis Sci $\underline{53}, 7539$

Lambert SR, Purohit A, Superak HM, Lynn MJ, Beck AD (2013): Long-term risk of glaucoma after congenital cataract surgery. Am J Ophthalmol 156, 355-361.e2

Lambert SR, Lynn MJ, Hartmann EE, DuBois L, Drews-Botsch C, Freedman SF, Plager DA, Buckley EG, Wilson ME (2014): Comparison of Contact Lens and Intraocular Lens Correction of Monocular Aphakia During Infancy: A Randomized Clinical Trial of HOTV Optotype Acuity at Age 4.5 Years and Clinical Findings at Age 5 Years. JAMA Ophthalmol 132, 676-682

Lambert SR, Plager DA, Buckley EG, Wilson ME, DuBois L, Drews-Botsch CD, Harmann EE, Lynn MJ (2015): The Infant Aphakia Treatment Study: Further on intra- and postoperative complications in the intraocular lens group. J AAPOS $\underline{19}, 101-103$

Lambert SR, Cotsonis G, DuBois L, Wilson ME, Plager DA, Buckley EG, McClatchey SK (2016): Comparison of the rate of refractive growth in aphakic eyes versus pseudophakic eyes in the Infant Aphakia Treatment Study. J Cataract Refract Surg 42, 1768-1773

Lapid-Gortzak R, Meulen IJ van der, Jellema HM, Mourits MP, Nieuwendaal CP (2016): Seven-year follow-up of unilateral multifocal pseudophakia in a child. Int Ophthalmol 1-4

Lim ME, Buckley EG, Prakalapakorn SG (2017): Update on congenital cataract surgery management: Curr Opin Ophthalmol 28, 87-92

Lim Z, Rubab S, Chan YH, Levin AV (2010): Pediatric Cataract: The Toronto Experience-Etiology. Am J Ophthalmol 149, 887-892

Lin H, Lin D, Chen J, Luo L, Lin Z, Wu X, Long E, Zhang L, Chen H, Chen W, et al. (2016): Distribution of Axial Length before Cataract Surgery in Chinese Pediatric Patients. Sci Rep $\underline{6}$, 23862

Lu Y, Ji Y-H, Luo Y, Jiang Y-X, Wang M, Chen X (2010): Visual results and complications of primary intraocular lens implantation in infants aged 6 to 12 months. Graefes Arch Clin Exp Ophthalmol $\underline{248}, 681-686$

Lüchtenberg M, Kohnen T (2007): Untersuchungsmethodik zur Diagnostik der ein- und beidseitigen kindlichen Katarakt. Ophthalmologe 104, 552-558

Lüchtenberg M, Kuhli-Hattenbach C, Fronius M, Zubcov AA, Kohnen T (2008): Predictability of Intraocular Lens Calculation Using the Holladay II Formula after in-the-Bag or Optic Captured Posterior Chamber Intraocular Lens Implantation in Paediatric Cataracts. Ophthalmologica 222, 302-307

Lundvall A, Kugelberg U (2002a): Outcome after treatment of congenital unilateral cataract. Acta Ophthalmol Scand $\underline{80}, 588-592$

Lundvall A, Kugelberg U (2002b): Outcome after treatment of congenital bilateral cataract. Acta Ophthalmol Scand $\underline{80}, 593-597$

Lundvall A, Zetterström C (2006): Primary intraocular lens implantation in infants: Complications and visual results. J Cataract Refract Surg $\underline{32}, 1672-1677$

Magli A, Forte R, Rombetto L (2013): Long-term outcome of primary versus secondary intraocular lens implantation after simultaneous removal of bilateral congenital cataract. Graefes Arch Clin Exp Ophthalmol 251, 309-314 
Magli A, Forte R, Carelli R, Magli G, Esposito F, Torre A (2016a): Long-Term Follow-Up after Surgery for Congenital and Developmental Cataracts. Semin Ophthalmol 31, 261-265

Magli A, Forte R, Carelli R, Rombetto L, Magli G (2016b): Long-Term Outcomes of Primary Intraocular Lens Implantation for Unilateral Congenital Cataract. Semin Ophthalmol 31, 548-553

Magli A, Carelli R, Forte R, Vecchio EC, Esposito F, Torre A (2017): Congenital and Developmental Cataracts: Focus on Strabismus Outcomes at Long-Term Follow-Up. Semin Ophthalmol $\underline{32}$, 358362

Mataftsi A, Dabbagh A, Moore W, Nischal KK (2012): Evaluation of whether intracameral dexamethasone predisposes to glaucoma after pediatric cataract surgery. J Cataract Refract Surg $\underline{38}, 1719-1723$

Mataftsi A, Haidich A-B, Kokkali S, Rabiah PK, Birch E, Stager DR, Cheong-Leen R, Singh V, Egbert JE, Astle WF, et al. (2014): Postoperative glaucoma following infantile cataract surgery: an individual patient data meta-analysis. JAMA Ophthalmol 132, 1059-1067

McClatchey SK, Dahan E, Maselli E, Gimbel HV, Wilson ME, Lambert SR, Buckley EG, Freedman SF, Plager DA, Parks MM (2000): A comparison of the rate of refractive growth in pediatric aphakic and pseudophakic eyes. Ophthalmology $\underline{107}, 118-122$

Merin S, Crawford JS (1971): The etiology of congenital cataracts. A survey of 386 cases. Can J Ophthalmol $\underline{6}, 178-182$

Moore KL, Persaud TVN: Embryologie: Entwicklungsstadien, Frühentwicklung, Organogenese, Klinik. 5. Auflage; Elsevier, Urban \& Fischer, München Jena 2007

Morcher Ophtalmic. http://www.morcher.com/en/products/bag-in-the-lens/background-of-thebil.html; Zugriff am 14.12.2016

Nischal KK, Medsinge A (2015): Pediatric cataract: challenges and future directions. Clin Ophthalmol $\underline{9}$, $77-90$

Noack C, Lagrèze WA (2008): Zur Behandlung der kindlichen Katarakt in Deutschland - Ergebnisse einer Umfrage. Klin Monatsbl Augenheilkd 225, 80-85

O'Keefe M, Fenton S, Lanigan B (2001): Visual outcomes and complications of posterior chamber intraocular lens implantation in the first year of life. J Cataract Refract Surg 27, 2006-2011

Papadopoulos M, Khaw PT (2003): Meeting the challenge of glaucoma after paediatric cataract surgery. Eye Lond Engl 17, 1-2

Papadopoulos M, Cable N, Rahi J, Khaw PT, BIG Eye Study Investigators (2007): The British Infantile and Childhood Glaucoma (BIG) Eye Study. Invest Ophthalmol Vis Sci 48, 4100-4106

Parks MM, Johnson DA, Reed GW (1993): Long-term Visual Results and Complications in Children with Aphakia. Ophthalmology 100, 826-841

Plager DA, Lynn MJ, Buckley EG, Wilson ME, Lambert SR (2011): Complications, Adverse Events, and Additional Intraocular Surgery 1 Year After Cataract Surgery in the Infant Aphakia Treatment Study. Ophthalmology 118, 2330-2334

Plager DA, Lynn MJ, Buckley EG, Wilson ME, Lambert SR, Plager DA, Lynn MJ, Buckley EG, Wilson ME, Lambert SR, et al. (2014): Complications in the First 5 Years Following Cataract Surgery in Infants With and Without Intraocular Lens Implantation in the Infant Aphakia Treatment Study. Am J Ophthalmol 158, 892-898.e2 
Pueringer SL, Hodge DO, Erie JC (2011): Risk of Late Intraocular Lens Dislocation After Cataract Surgery, 1980-2009: A Population-Based Study. Am J Ophthalmol 152, 618-623

Raina UK, Bhambhwani V, Gupta A, Bhushan G, Seth A, Ghosh B (2016): Comparison of Transcorneal and Pars Plana Routes in Pediatric Cataract Surgery in Infants Using a 25-Gauge Vitrectomy System. J Pediatr Ophthalmol Strabismus $\underline{\text { 53, }}$ 105-112

Rogers GL, Tishler CL, Tsou BH, Hertle RW, Fellows RR (1981): Visual acuities in infants with congenital cataracts operated on prior to 6 months of age. Arch Ophthalmol 1960 99, 999-1003

Rong X, Ji Y, Fang Y, Jiang Y, Lu Y (2015): Long-Term Visual Outcomes of Secondary Intraocular Lens Implantation in Children with Congenital Cataracts. PLOS ONE 10, e0134864

Sadoughi MM, Einollahi B, Roshandel D, Sarimohammadli M, Feizi S (2015): Visual and Refractive Outcomes of Phacoemulsification with Implantation of Accommodating versus Standard Monofocal Intraocular Lenses. J Ophthalmic Vis Res $\underline{10}, 370-374$

Simon JW, Mehta N, Simmons ST, Catalano RA, Lininger LL (1991): Glaucoma after pediatric lensectomy/vitrectomy. Ophthalmology $\underline{98}, 670-674$

Sminia ML, de Faber JT, Doelwijt DJ, Wubbels RJ, Tjon-Fo-Sang M (2010): Axial eye length growth and final refractive outcome after unilateral paediatric cataract surgery. Br J Ophthalmol $\underline{94}, 547-550$

Solebo AL, Russell-Eggitt I, Cumberland PM, Rahi JS (2015): Risks and outcomes associated with primary intraocular lens implantation in children under 2 years of age: the IoLunder2 cohort study. $\mathrm{Br} \mathrm{J}$ Ophthalmol 99, 1471-1476

Stager DR, Weakley DR, Hunter JS (2002): Long-term rates of PCO following small incision foldable acrylic intraocular lens implantation in children. J Pediatr Ophthalmol Strabismus $\underline{39}$, 73-76

Struck MC (2015): Long-term Results of Pediatric Cataract Surgery and Primary Intraocular Lens Implantation From 7 to 22 Months of Life. JAMA Ophthalmol 133, 1180-1183

Sukhija J, Ram J, Gupta N, Sawhney A, Kaur S (2014): Long-term results after primary intraocular lens implantation in children operated less than 2 years of age for congenital cataract. Indian J Ophthalmol $\underline{62}, 1132$

Superstein R, Archer SM, Del Monte MA (2002): Minimal myopic shift in pseudophakic versus aphakic pediatric cataract patients. J AAPOS $\underline{6}, 271-276$

Tadros D, Trivedi RH, Wilson ME, Davidson JD (2016): Ocular axial growth in pseudophakic eyes of patients operated for monocular infantile cataract: a comparison of operated and fellow eyes measured at surgery and 5 or more years later. J AAPOS $\underline{20}, 210-213$

Taruscio D, Capozzoli F, Frank C (2011): Rare diseases and orphan drugs. Ann Ist Super Sanita 47, 83-93

Taylor D, Vaegan null, Morris JA, Rodgers JE, Warland J (1979): Amblyopia in bilateral infantile and juvenile cataract. Relationship to timing of treatment. Trans Ophthalmol Soc U K $\underline{99}, 170-175$

Trivedi RH, Wilson ME (2007a): Biometry Data from Caucasian and African-American Cataractous Pediatric Eyes. Invest Opthalmol Vis Sci $\underline{48}, 4671$

Trivedi RH, Wilson ME (2007b): Changes in interocular axial length after pediatric cataract surgery. J AAPOS $\underline{11}, 225-229$

Trivedi RH, Wilson ME, Golub RL (2006): Incidence and Risk Factors for Glaucoma After Pediatric Cataract Surgery With and Without Intraocular Lens Implantation. J AAPOS 10, 117-123 
Trivedi RH, Wilson ME, Vasavada AR, Shah SK, Vasavada V, Vasavada VA (2011): Visual axis opacification after cataract surgery and hydrophobic acrylic intraocular lens implantation in the first year of life. J Cataract Refract Surg 37, 83-87

Van Looveren J, Ní Dhubhghaill S, Godts D, Bakker E, De Veuster I, Mathysen DGP, Tassignon M-J (2015): Pediatric bag-in-the-lens intraocular lens implantation: Long-term follow-up. J Cataract Refract Surg 41, 1685-1692

van Noorden GK (1978): [Clinical aspects of deprivation amblyopia (author's transl)]. Klin Monatsbl Augenheilkd 173, 464-469

VanderVeen DK, Trivedi RH, Nizam A, Lynn MJ, Lambert SR (2013): Predictability of Intraocular Lens Power Calculation Formulae in Infantile Eyes With Unilateral Congenital Cataract: Results from the Infant Aphakia Treatment Study. Am J Ophthalmol 156, 1252-1260.e2

Vasavada AR, Raj SM, Nihalani B (2004): Rate of axial growth after congenital cataract surgery. Am J Ophthalmol 138, 915-924

Weakley DR, Birch E, McClatchey SK, Felius J, Parks MM, Stager D (2003): The association between myopic shift and visual acuity outcome in pediatric aphakia. J AAPOS $\underline{7}, 86-90$

Weakley DR, Lynn MJ, Dubois L, Cotsonis G, Wilson ME, Buckley EG, Plager DA, Lambert SR (2017): Myopic Shift 5 Years after Intraocular Lens Implantation in the Infant Aphakia Treatment Study. Ophthalmology 124, 822-827

Whitman MC, Vanderveen DK (2014): Complications of pediatric cataract surgery. Semin Ophthalmol $\underline{29}$, $414-420$

Wiesel TN, Hubel DH (1963): Effects of Visual Deprivation on Morphology and Physiology of Cells in the Cat's Lateral Geniculate Body. J Neurophysiol 26, 978-993

Wilson ME, Trivedi RH (2007): Choice of intraocular lens for pediatric cataract surgery: Survey of AAPOS members. J Cataract Refract Surg $\underline{33}$, 1666-1668

Wilson ME, Trivedi RH, Weakley DR, Cotsonis GA, Lambert SR (2017): Globe Axial Length Growth at Age 5 Years in the Infant Aphakia Treatment Study. Ophthalmology 124, 730-733

Wu X, Long E, Lin H, Liu Y (2016): Prevalence and epidemiological characteristics of congenital cataract: a systematic review and meta-analysis. Sci Rep $\underline{6}, 28564$

Zetterström C, Lundvall A, Kugelberg M (2005): Cataracts in children. J Cataract Refract Surg 31, 824840

Zhang L, Wu X, Lin D, Long E, Liu Z, Cao Q, Chen J, Li X, Lin Z, Luo L, et al. (2016): Visual Outcome and Related Factors in Bilateral Total Congenital Cataract Patients: A Prospective Cohort Study. Sci Rep $\underline{6}$, srep31307

Zhou H-W, Zhou F (2016): A Meta-analysis on the clinical efficacy and safety of optic capture in pediatric cataract surgery. Int J Ophthalmol $\underline{9}, 590-596$ 


\section{Danksagung}

Ich bedanke mich ganz herzlich bei Herrn Prof. Dr. med. N. Feltgen für die freundliche Überlassung dieses interessanten Themas sowie die konstante und enge Betreuung und Beratung bei der Ausarbeitung.

Mein besonderer Dank gilt Herrn Dr. med. J. Callizo für die über die Maßen engagierte und intensive Betreuung und Begleitung bei der Konzeption und Durchführung dieser Untersuchung, für die wertvollen Anregungen bei der Erstellung dieser Dissertationsschrift sowie für die konstruktive Kritik und die Unterstützung, die das Erstellen dieser Arbeit zu etwas Besonderem haben werden lassen.

Daneben gilt mein Dank allen Mitarbeitern der verschiedenen Abteilungen der Universitätsmedizin Göttingen, die durch ihre freundliche und hilfsbereite Art zum Fortkommen dieser Arbeit beigetragen haben. 